\title{
WestVirginiaUniversity
}

THE RESEARCH REPOSITORY @ WVU

Graduate Theses, Dissertations, and Problem Reports

2007

\section{Modeling the fiberglass spinning process}

Jeremy S. Watts

West Virginia University

Follow this and additional works at: https://researchrepository.wvu.edu/etd

\section{Recommended Citation}

Watts, Jeremy S., "Modeling the fiberglass spinning process" (2007). Graduate Theses, Dissertations, and Problem Reports. 1817.

https://researchrepository.wvu.edu/etd/1817

This Thesis is protected by copyright and/or related rights. It has been brought to you by the The Research Repository @ WVU with permission from the rights-holder(s). You are free to use this Thesis in any way that is permitted by the copyright and related rights legislation that applies to your use. For other uses you must obtain permission from the rights-holder(s) directly, unless additional rights are indicated by a Creative Commons license in the record and/ or on the work itself. This Thesis has been accepted for inclusion in WVU Graduate Theses, Dissertations, and Problem Reports collection by an authorized administrator of The Research Repository @ WVU. For more information, please contact researchrepository@mail.wvu.edu. 


\title{
Modeling the Fiberglass Spinning Process
}

\author{
Jeremy S. Watts \\ Thesis Submitted to the \\ College of Engineering \\ At West Virginia University \\ Master of Science \\ In \\ Mechanical Engineering \\ Larry Banta, Ph.D, Chair \\ Ken Means, Ph.D \\ Eric Johnson, Ph.D \\ Dept. of Mechanical Engineering \\ Morgantown, WV \\ 2007
}

In Partial Fulfillment of the Requirements for the Degree of

Keywords: Fiberglass, Viscoelasticity, Heat Transfer 


\section{Abstract \\ Modeling the Fiber Glass Spinning Process}

\section{Jeremy Watts}

Two main processes are performed in the manufacturing of fiberglass filters. This thesis looks at the first of these processes, which is known as the spinning process in the industry. At the subject plant, melted glass is drawn from a moving furnace through a plate with several \#16 sized holes acting as tiny nozzles and spun onto a large-diameter drum spinning at a set speed. The glass strands after being drawn have an average diameter of 27-30 microns. While the glass is being spun on the drum, a special binder is applied to the glass strands that will later be used to bond the glass together and give the final product dimensional stability. The glass is spun until a certain weight is achieved. Then it is cut and removed in the form of an $18 \mathrm{ft}$. mat, and sent to the second stage of production. Currently, the system's controlled variables (temperatures, furnace speed, drum speed, etc.) are just combinations derived experimentally by the subject company to produce certain products.

The glass being drawn from the furnace occasionally breaks, causing problems in the process, along with down time. The furnace motor does not disengage when the sensor reads an error, so the furnace continues to traverse the drum as if nothing is wrong. This means there is a potential for missed steps such as a place where two fast passes of the furnace are supposed to occur. It can also lead to portions of the mat being thicker in some places than in others. It is unknown whether these small inconsistencies have a large effect on the amount of waste generated in the expansion process or in the finished product itself.

This thesis uses theoretical formulas to create a working model of the process. From here, different controllable variables will be changed and analyzed to determine their effect on the final product. This can help determine an effective way to improve production in the future.

Committee Members: Dr. Larry Banta (chair), Dr. Ken Means, Dr. Eric Johnson 


\section{Acknowledgements}

I would like to thank my family and friends for their encouragement not to quit while writing this thesis, everyone who has ever been proud of me for making it this far. I would also like to thank my committee members, Dr. Kenneth Means and Dr. Eric Johnson, for their time spent reading my thesis and giving me feedback at my defense. Most of all I would like to thank my committee chairman, Dr. Larry Banta, for putting up with me for so long, reading, commenting and revising my atrocious grammar, and acting as my liaison with the faculty of West Virginia University while I was thousands of miles away in Texas. Without him I would have failed at something as minuscule as the paperwork, not to mention the actual thesis. 


\section{Table of Contents}

Modeling the Fiberglass Spinning Process .................................................................................. i

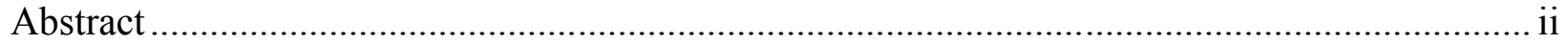

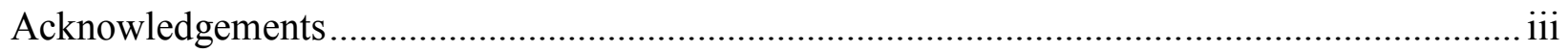

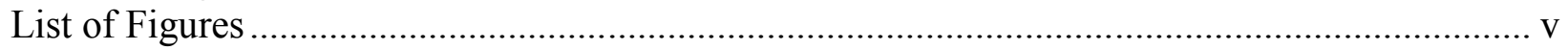

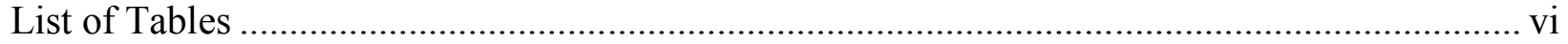

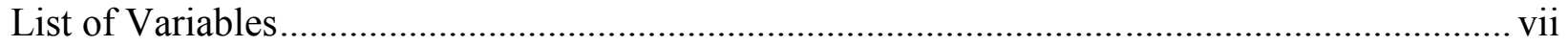

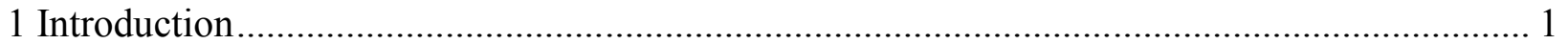

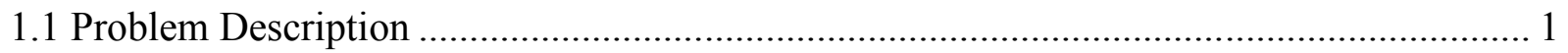

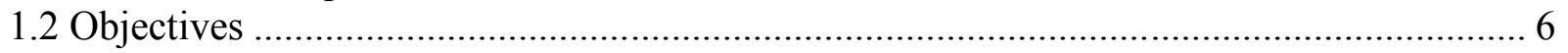

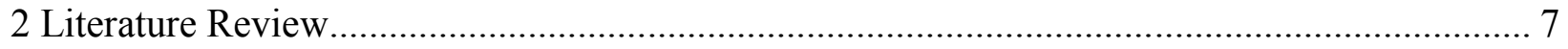

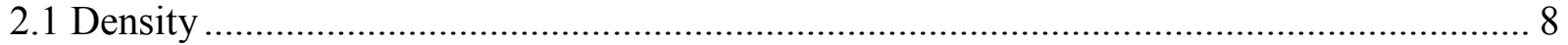

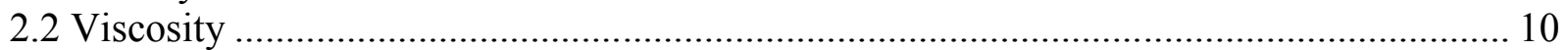

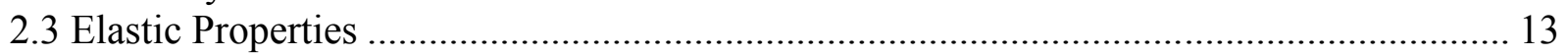

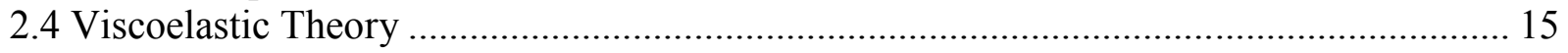

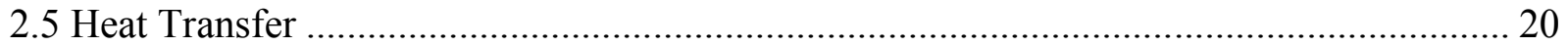

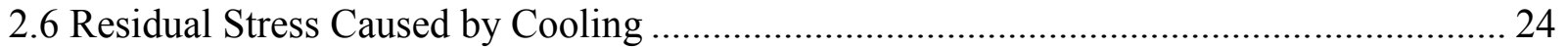

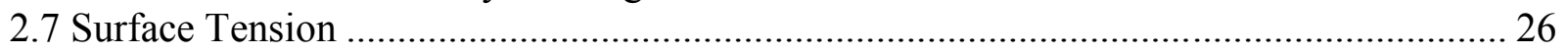

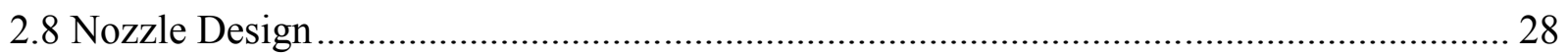

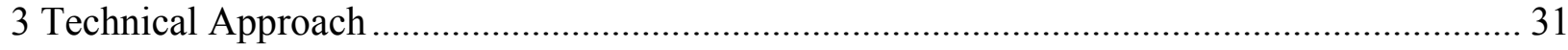

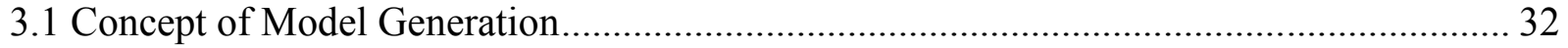

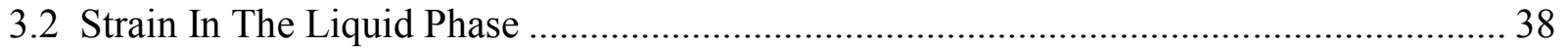

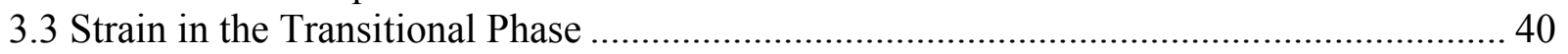

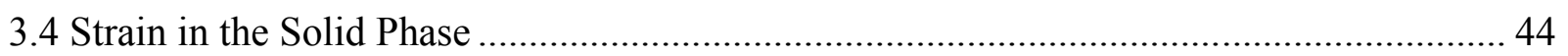

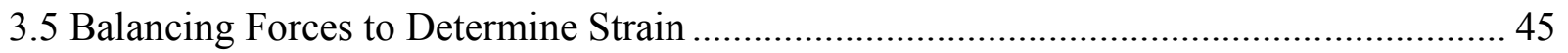

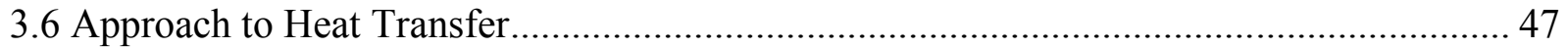

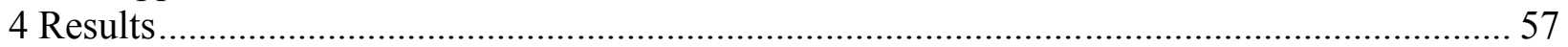

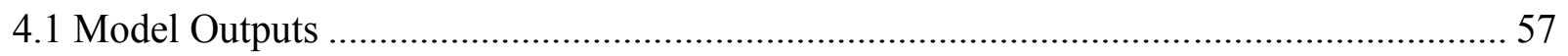

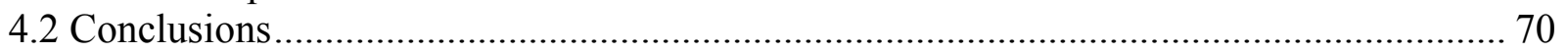

4.3 Suggestions for Higher Efficiency ............................................................................ 71

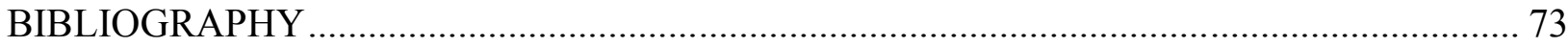

Appendix 1 Determining Strand Diameter ........................................................................... 75

Appendix 2 Modeling the Attenuation of Fiber Glass ................................................................. 77

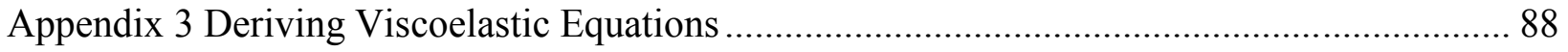




\section{List of Figures}

Table 2.5.1 Kinematic Viscosity of Air, reproduced from Mills, p. 920 [4] 20 ......................... vi

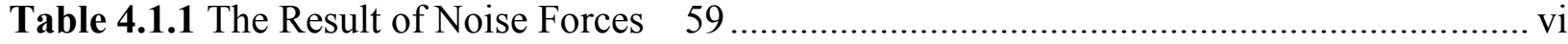

Figure 1.1.1 Cross-sectional view of Nozzle .......................................................................... 1

Figure 1.1.2 Illustration of the basic set-up of the system ...................................................... 3

Figure 1.1.3 Picture of "sling" covering parts of the machine................................................... 5

Figure 2.1.1: Density of soda lime glass as a function of temperature........................................ 8

Figure 2.2.1 The Viscosity of Glass as a Function of Temperature........................................... 11

Figure 2.3.1 Young's Modulus as a Function of Temperature ……………………………......... 13

Figure 2.4.1 Maxwell Model and its Creep Test Response ................................................. 16

Figure 2.4.2 Maxwell Representation and Relaxation Test Results .......................................... 17

Figure 2.4.3 Kelvin Representation and Creep Test Response.................................................. 18

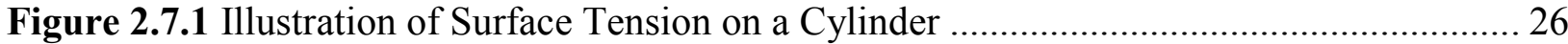

Figure 3.1.1: Illustration of the first control volume fixed in space ........................................ 34

Figure 3.1.2 Illustration of Continual Control Volume Generation.............................................. 36

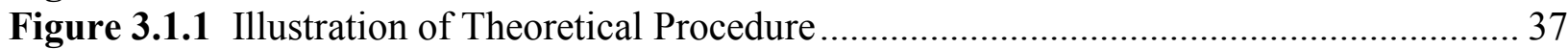

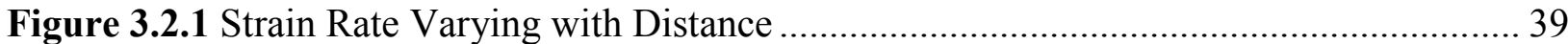

Figure 3.3.1 Three-parameter Fluid Representation and Creep Test Response............................ 42

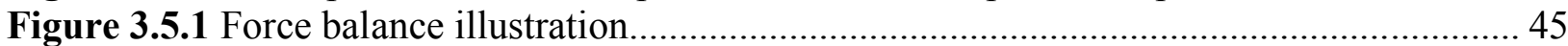

Figure 3.6.1 Illustration of Energy Flow in a Strand Section .................................................. 48

Figure 3.6.2 Illustration of Radiation Viewed Parallel to Strand Axis ........................................ 49

Figure 3.6.3 Radiation from a View Perpendicular to the Strand Axis ......................................5 50

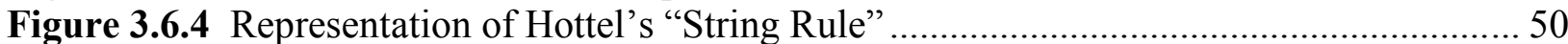

Figure 4.1.1 Strand Diameter as a Function of Distance from the Furnace..................................5 58

Figure 4.1.2 View of nozzle plate, coils, and glass meniscus...................................................5 58

Figure 4.1.3 Cross-Section Temperature as a Function of Distance (assuming cross-section temperatures constant based on Biot Numbers).................................................................5 59

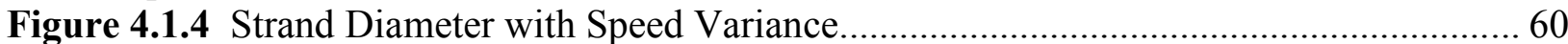

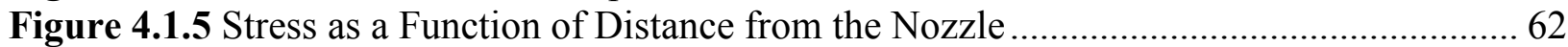

Figure 4.1.6 Stress as a Function of RPM at Three Varying Ambient Temperatures .................. 63

Figure 4.1.7 Stress as a Function of Ambient Temperature....................................................... 64

Figure 4.1.8 Theoretical Meniscus Base Diameter as a Function of Time to Illustrate Effect of

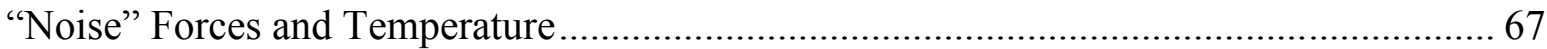

Figure 4.1.9 Stress as a Function of Glass Temperature inside the Furnace ...............................6 68 


\section{List of Tables}

Table 2.5.1 Kinematic Viscosity of Air, reproduced from Mills, p. 920 [4] ........................... 21

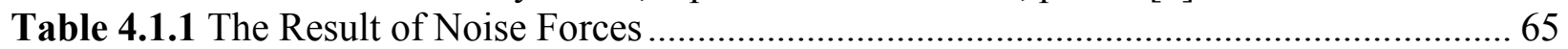




\section{List of Variables}

\begin{tabular}{ll} 
A & Area \\
$\mathrm{C}_{\mathrm{p}}$ & Specific Heat \\
$\mathrm{D}$ & Diameter \\
$\mathrm{E}$ & Young's Modulus \\
$\mathrm{g}$ & Gravitational Constant \\
$\mathrm{h}$ & Heat Transfer Coefficient / Furnace \\
$\mathrm{k}$ & Height \\
$\mathrm{L}$ & Thermal Conductivity \\
$\mathrm{m}$ & Length \\
$\mathrm{Nu}$ & Mass \\
$\mathrm{Pr}$ & Nusselt Number \\
$\mathrm{Q}, \mathrm{q}$ & Prandtl Number \\
$\mathrm{R}$ & Heat Transfer \\
$\mathrm{Re}$ & Radius \\
$\mathrm{T}$ & Reynold's Number \\
$\mathrm{t}$ & Temperature \\
$\mathrm{V}$ & time \\
$\mathrm{X}$ & Velocity \\
$\varepsilon$ & Distance from Furnace \\
$\eta$ & Emisivity / Strain \\
$\lambda$ & Viscosity \\
$\rho$ & Coefficient of Viscous Traction \\
$\sigma$ & Density \\
$\tau$ & Stefan-Boltzmann constant / Stress \\
$v$ & Stress \\
$\mathrm{p}$ & Kinematic Viscosity of Air \\
$\mathrm{Y}$ & Pressure \\
& Surface Tension \\
& \\
\hline
\end{tabular}




\section{Introduction}

\subsection{Problem Description}

Two main processes are performed in the manufacturing of fiberglass filters. This thesis looks at the first of these processes, which is known as the spinning process in the industry. At the subject plant, melted glass is drawn from a moving furnace through a plate with several \#16 sized holes acting as tiny nozzles and spun onto a drum 18 feet in circumference, spinning at about 149 RPM. In metric units the diameter of the nozzles is $0.00427 \mathrm{~m}$ or $4.27 \mathrm{~mm}$. The centers of the nozzles are spaced approximately $8 \mathrm{~mm}$ apart in a box pattern. There are two different variations on the number of nozzles on a plate. One such variation uses two rectangular shaped grids with 21 nozzles along its length and 7 nozzles along its width. This gives a total of 294 nozzles. The two rectangular grids are approximately twenty-four and a half millimeters apart, measured from the centers of the nozzles on the edge of each rectangle.

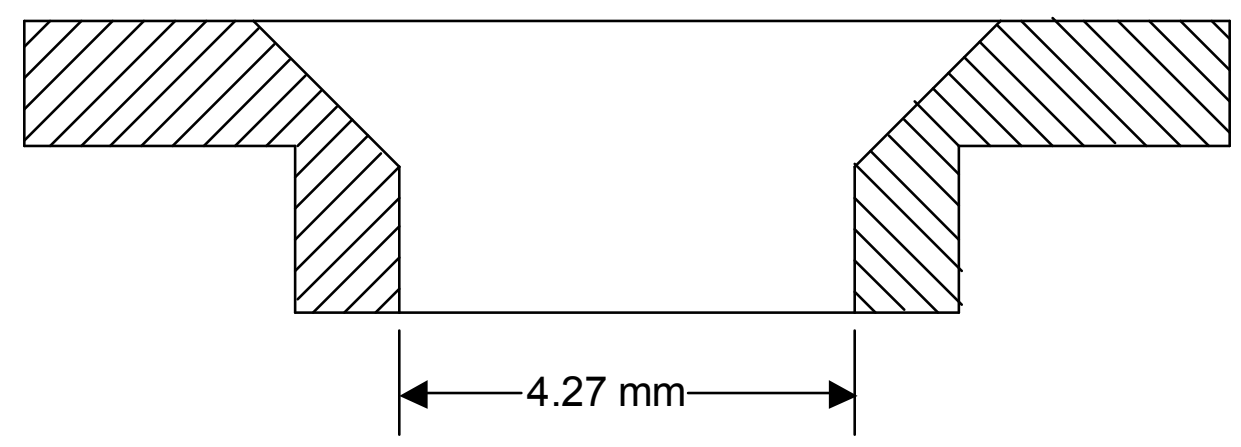

Figure 1.1.1 Cross-sectional view of Nozzle 
Under the furnace is a hood extending 0.1 meters below the plate containing the nozzles. Also inside the hood are liquid cooled coils that aid in the process of cooling the strands as they exit the furnace.

The current drawing speed of the drums is $149 \mathrm{rpm}$. At this speed the glass strands being drawn from the furnace have an average diameter of 27-30 microns. This will become the desired diameter range for the product.

As the glass is being drawn from the furnace, the furnace is moving back and forth along the axis of the drum. The traverse speed of the furnace is varied to create different angles for the strands in the mat. For example, a strand being drawn from a non-traversing furnace would be wound around the drum at an angle perpendicular to the drum's axis, whereas a fast traversing furnace could create an acute angle between the strand and the axis. Using different combinations of traversing speeds controls the expansion of the mat and distinguishes different products made by the company.

While the glass is being spun on the drum, a special binder is applied to the glass strands that will later be used to bond the glass together and give the final product dimensional stability. The glass is spun until a certain weight is achieved, and is then cut and removed in the form of a mat 18 feet wide by the length of the drum. The weight of the mat determines its thickness, another characteristic that distinguishes different products. The length of this mat varies between different drums, because the company has several different sized drums throughout the plant. The drums however, are all the same diameter. The mat is wrapped around a long bar and sent to the second stage of production known as the expansion process. 


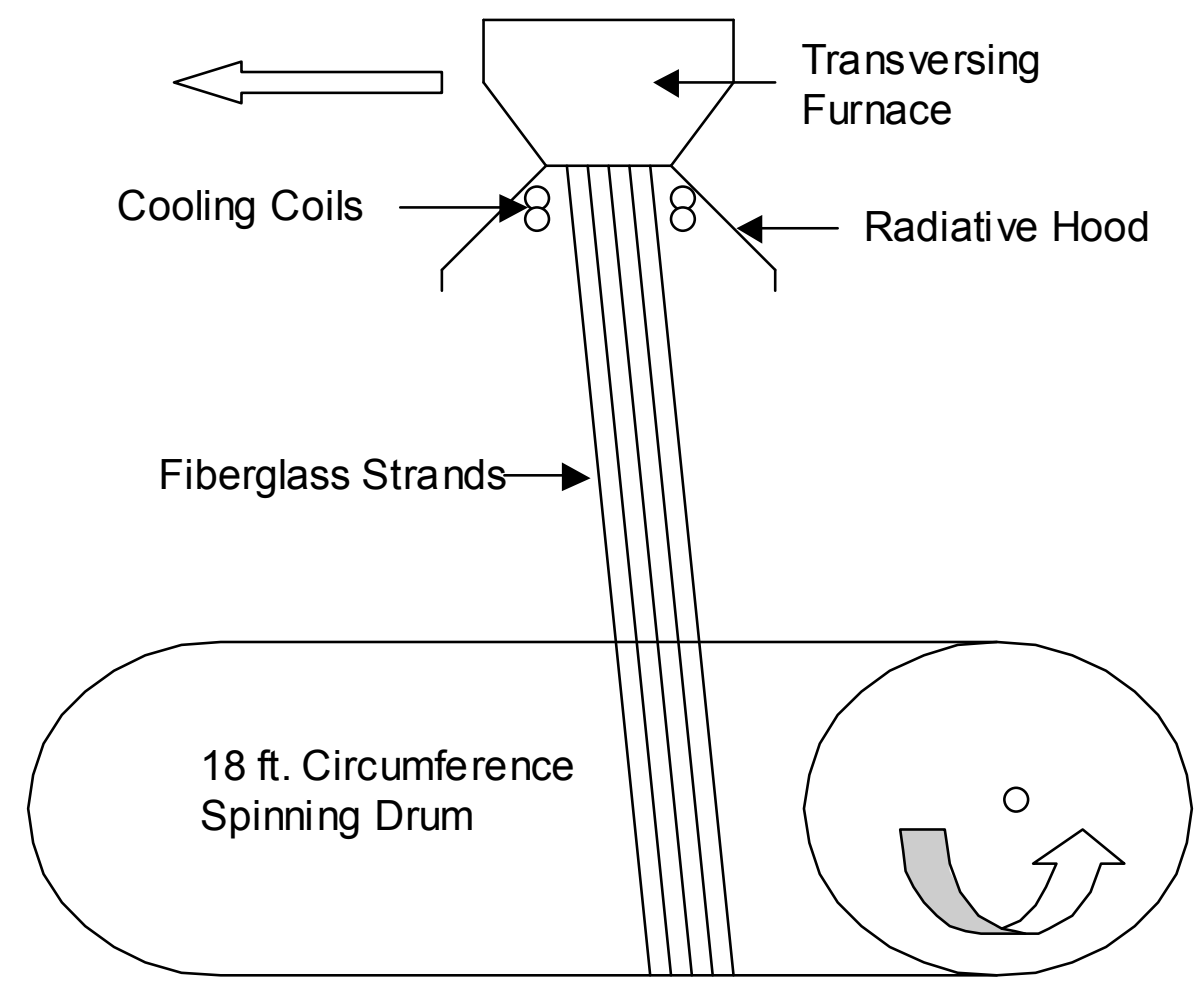

Figure 1.1.2 Illustration of the basic set-up of the system

Currently, the system's controlled variables (temperatures, furnace speed, drum speed, etc.) are combinations that have been derived experimentally by the subject company to produce certain products. After the expansion process sample pieces are taken from the finished mat and analyzed to insure consistency with the product specifications.

The process for spinning the correct amount of glass onto the drums is rarely a steady state process. On occasion, one or more strands will break. When glass strands break and are no longer being drawn onto the drum, the plate starts to heat due to the lack of heat transfer from the strands to the air. A heat sensor monitors the temperature of the plate below the furnace that the glass is drawn through. At a certain increased temperature the system controller signals an error. The furnace motor does not disengage when the sensor reads an error, so the furnace continues to 
traverse the drum as if nothing is wrong. At this point a worker has to manually pull strands from the furnace and restart the spinning process onto the drum. When the worker restarts the process some of the glass is disposed of on the floor around the machinery where it will later have to be removed. This glass cannot be recycled and becomes waste product. This also causes a minute or two of down time. Because of the downtime and the continuing movement of the furnace, the mat has a potential for missed steps (such as a place where two fast passes of the furnace are supposed to occur). It can also lead to portions of the mat being thicker in some places than in others. It is unknown whether these small inconsistencies have a large effect on the amount of waste generated in the expansion process or in the finished product itself.

One major part of the spinning process is the application of the binder to the glass strands. The company that manufactures the binder performs lab tests on it; however, the company's data does not include information on the viscosity and tackiness of the binder under various atmospheric and temperature conditions. This is important because employees observe that the drums "sling" binder more on a hot, humid day as compared to a cold, winter day. One theory on why the glass strands break is that airborne binder has a tendency to strike the glass strands as they are drawn from the furnace. Being able to determine a drum speed that allows the binder to remain on the drums under various ambient conditions is important in the overall control of system. If slinging binder is found to be a direct cause of breakage, the ability to create the exact same product at a speed that would lower the occurrence of binder slinging would prove beneficial when trying to eliminate downtime. 


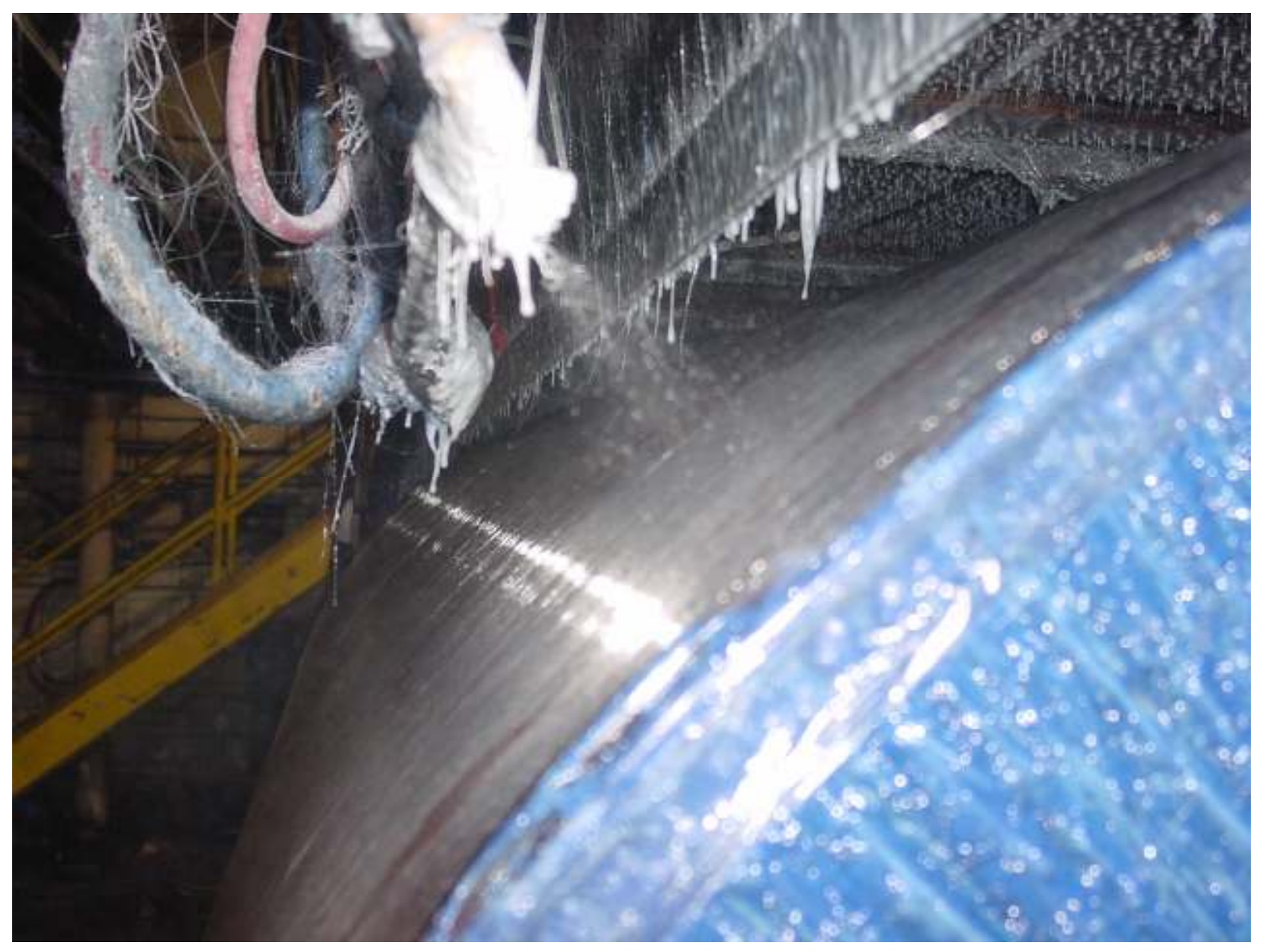

Figure 1.1.3 Picture of "sling" covering parts of the machine

Other theories of why the strands break, especially on hotter days, deal with heat transfer. It is possible that higher ambient temperatures cause a significant difference in the rate at which heat is transferred from the drawn glass to the atmosphere leaving the glass too hot and therefore less viscous as it is drawn from the furnace. Less viscous liquids, like oil or water, deform with less resistance than more viscous liquids, such as honey.

The motivation for the research is to improve the reliability of the process and in turn the quality of the final product. When one of the glass strands breaks, it leaves the machine incapable of continuing with its job until a factory worker has time to restart the spinning process, leaving inconsistencies in the product. The problem that is faced is turning the 
individual processes for each product into a more efficiently controlled system that can take into account certain physical conditions and minimize waste as well as downtime. Obviously if the system were better controlled, the company would save a substantial amount of money that is otherwise lost.

\subsection{Objectives}

There are three main objectives of the research:

1. Develop mathematical models of the spinning process and its associated equipment

2. Analyze relationships among process variables and develop improved control strategies for spinning process

3. Present findings to plant engineers and propose experiments to test improved control algorithms 


\section{Literature Review}

The area of most concern when developing a mathematical model of the spinning process occurs between when the glass leaves the nozzle and when it is incorporated into the mat. During this time tensile stress acts on the flowing glass pulling it toward the drum and causing the glass a significant amount of strain. As the glass cools, physical properties of the glass change and it experiences three phase changes.

The glass is in liquid form as it exits the furnace. As the glass cools it begins to adopt characteristics of a solid combined with a liquid, also known as viscoelastic fluid. At a certain point the glass can finally be considered a solid. The glass leaves the nozzle with a rather large diameter in comparison to its final form, starting at $4.27 \mathrm{~mm}$ and ending around 27-30 microns. Understanding the physical properties of glass during this transition is essential when developing a model of the behavior of the glass.

The raw material used by the subject plant is remelted cullet from another company that produces light bulbs. It is a soda-lime silica glass and for most purposes will be compared with Corning Code 0800 glass. Most physical properties of soda-lime silica glass are temperature dependent, therefore it is important for the model to determine heat loss from the strands and ultimately develop a temperature profile. 


\subsection{Density}

The density of glass depends upon on its chemical composition. Since glass is not manufactured and only one type of glass is used at the subject company, all density measurements for Soda-Lime Silica glass will be used. The density of glass is related to temperature through the volume thermal expansion coefficient. The graph in Appendix A-2 shows the density of Soda-Lime Silica glass as a function of temperature. The graph is reproduced from "Handbook of Glass Manufacture" by Fay V. Tooley on page 920 [2]. Points were pulled from the original graph and entered into a spreadsheet to create a new graph. The graph in Tooley [2] and Figure 2.1.1 consist of two linear sections.

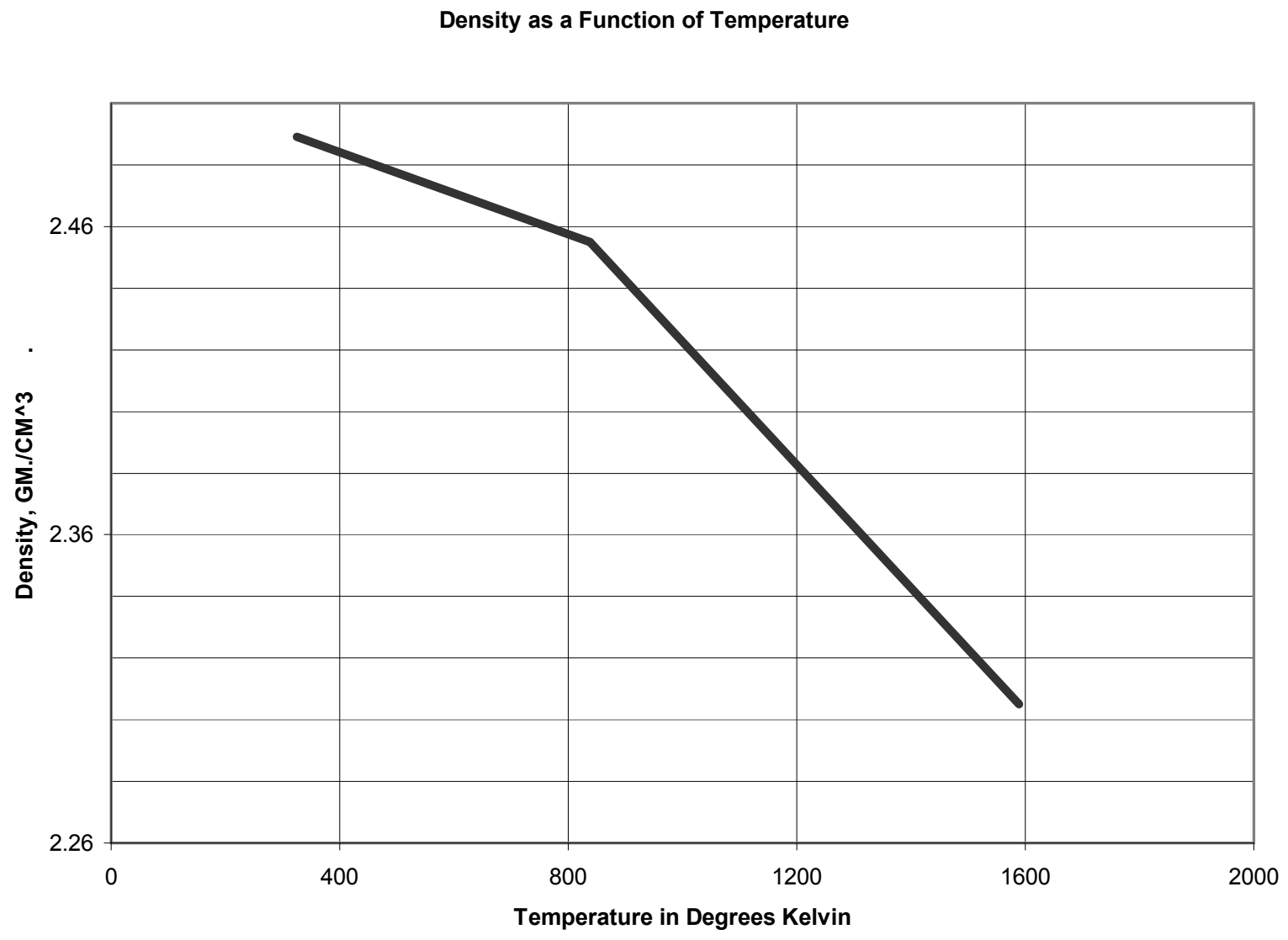

Figure 2.1.1: Density of soda lime glass as a function of temperature 
As can be seen in the graph, the density is piecewise linear with respect to temperature. The graph indicates an increase in the volume thermal expansion coefficient at approximately $850 \mathrm{~K}$. The change in the thermal expansion coefficient corresponds to the temperature at which the viscosity of glass passes the working point. The final graph takes into account density at the beginning of every step during the creation of the control volume fixed in space. This will be explained in greater detail in the technical approach chapter. 


\subsection{Viscosity}

Viscosity is a property that relates the shear stress acting on a fluid to the rate of deformation of a differential fluid volume. According to Varshneya on page 183 [3], these two properties are related by Newton's law of viscosity:

$$
\tau=\eta \dot{\varepsilon}
$$

where $\eta$ is the coefficient of viscosity. This coefficient is written in terms of $\mathrm{ML}^{-1} \mathrm{~T}^{-1}$. To keep units consistent throughout the model, the units of viscosity should be the same as the units used for stress multiplied by time. For example, if stress is measured in Pascals then the units of viscosity would be measured in Pa $\cdot \mathrm{s}$, or more commonly a poise (10 poise $=1 \mathrm{~Pa} \cdot \mathrm{s})$.

The viscosity of glass is highly dependent on temperature. Glass at lower temperatures tends to be more viscous than the same glass at higher temperatures as can be seen in Figure 2.2.1. The plot given here is reproduced from a graph in "Handbook of Glass Manufacture" by Fay V. Tooley on page 934 [2]. Points were taken from the original source and entered in a spreadsheet program to allow further analysis such as curve fit equations not given in the original source. The graph also displays four standard viscosity reference points: the strain point (approx. $3.2 \times 10^{14}$ poise), the annealing point (approx. $1 \times 10^{13}$ poise), the softening point (approx. $4 \times 10^{7}$ poise), and the working point $\left(1 \times 10^{4}\right.$ poise $)$. 
Viscosity as a Function of Temperature

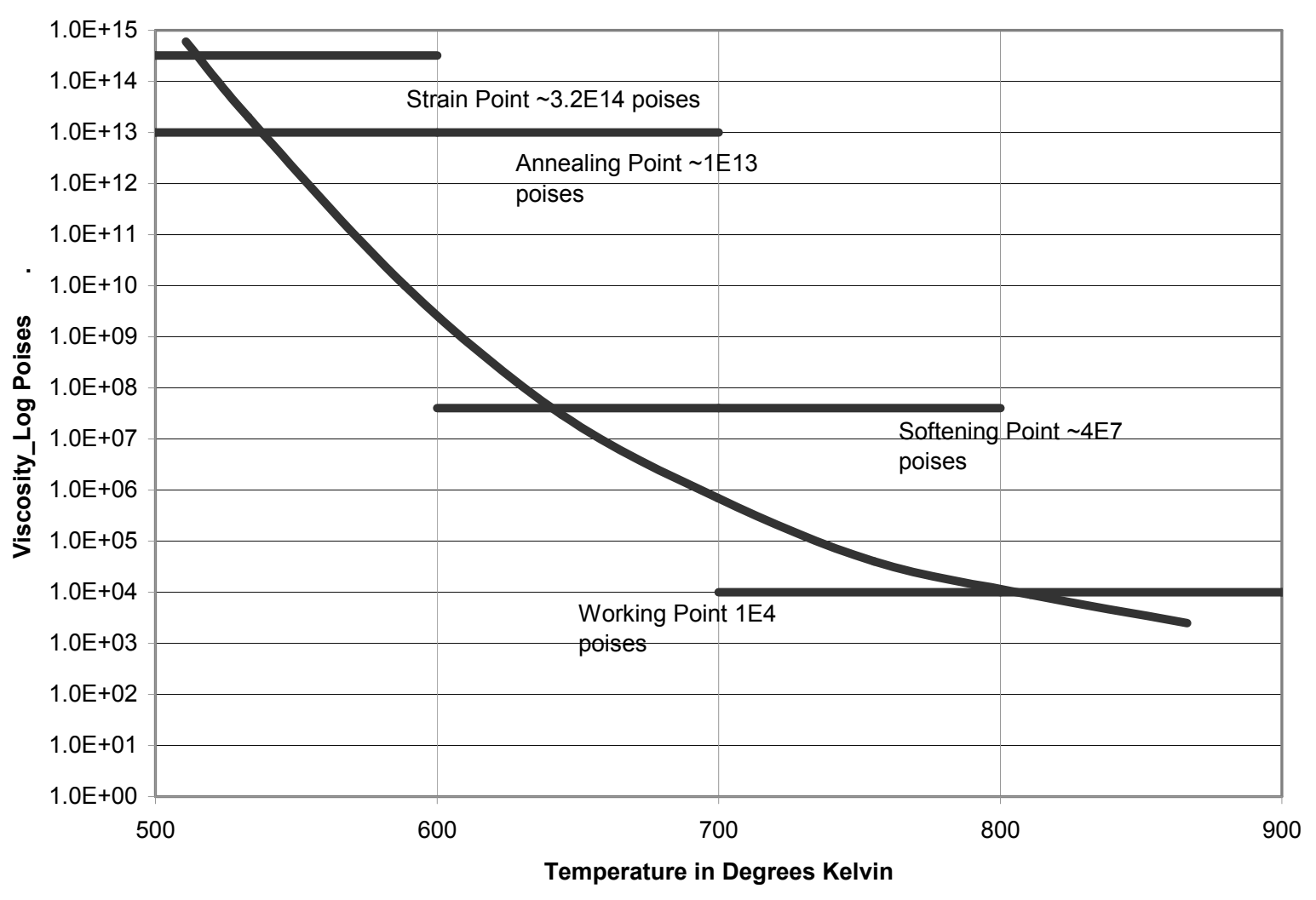

Figure 2.2.1 The Viscosity of Glass as a Function of Temperature

"The softening point of glass is defined by Littleton as the temperature at which a glass fiber $0.55-0.75 \mathrm{~mm}$ diameter and $23.5 \mathrm{~cm}$ long, of which the top $10 \mathrm{~cm}$ are heated at a rate of $5^{\circ} \mathrm{C} / \mathrm{min}$ when suspended vertically in a furnace of specified characteristics, elongates under its own weight at a rate of $1 \mathrm{~mm} / \mathrm{min}$. (Varshneya, 189-190) [3]" The annealing and strain points concern themselves with the process of releasing stresses caused while forming. The softening point can be defined as the viscosity at which glass can stand on its own and not be deformed by its own weight. 
The lowest temperature value given by the data is 510 degrees Kelvin. At temperatures below $510 \mathrm{~K}$ the amount of force needed to generate viscous deformation of the strand would be far greater than is present, and the material behaves and is treated by the model as a solid.

A unique characteristic of glass is that its solid form does not crystallize like metal or salts, but takes on a state similar to a liquid with extremely high viscosity. "The possibility of cooling molten glass to room temperature without crystallization is a result of its high viscosity which tends to prevent devitrification. (Tooley, 933)[2]” However, glass does not flow in response to stress at room temperature and can therefore be considered a solid.

During the fiberglass spinning process a force is being applied uniaxially on a strand of glass, giving the need to relate uniaxial stress to shear stress. One of Varshneya's [3] resources was able to do that with the following equation. "Trouton defined $\lambda$, the coefficient of viscous traction, through the equation:

$$
\sigma_{x}=\lambda \dot{e}_{x}
$$

and showed that $\lambda$ is approximately equal to $3 \eta$ (Varshneya, 184) [3]." Varshneya [3] also states that when using this equation in a multidimensional calculation, the Poisson ratio of glass must be changed from 0.22 to 0.5 . 


\subsection{Elastic Properties}

Glass in its solid form displays elastic properties similar to other solids like metals.

When stress is applied to glass at low temperatures, glass is considered to be elastic. Like most properties of glass, elastic properties are also highly dependent upon temperature and chemical composition. Elastic materials display a linear relationship between stress and uniaxial strain. Since the process uses a spinning drum to apply force and attenuate the strand of glass only uniaxial stresses will be needed in the model. The ratio of linear stress and strain, or Young's Modulus, of glass is dependent on temperature as can be seen in the graph in Figure 2.3.1.

Young's Modulus as a Function of Temperature

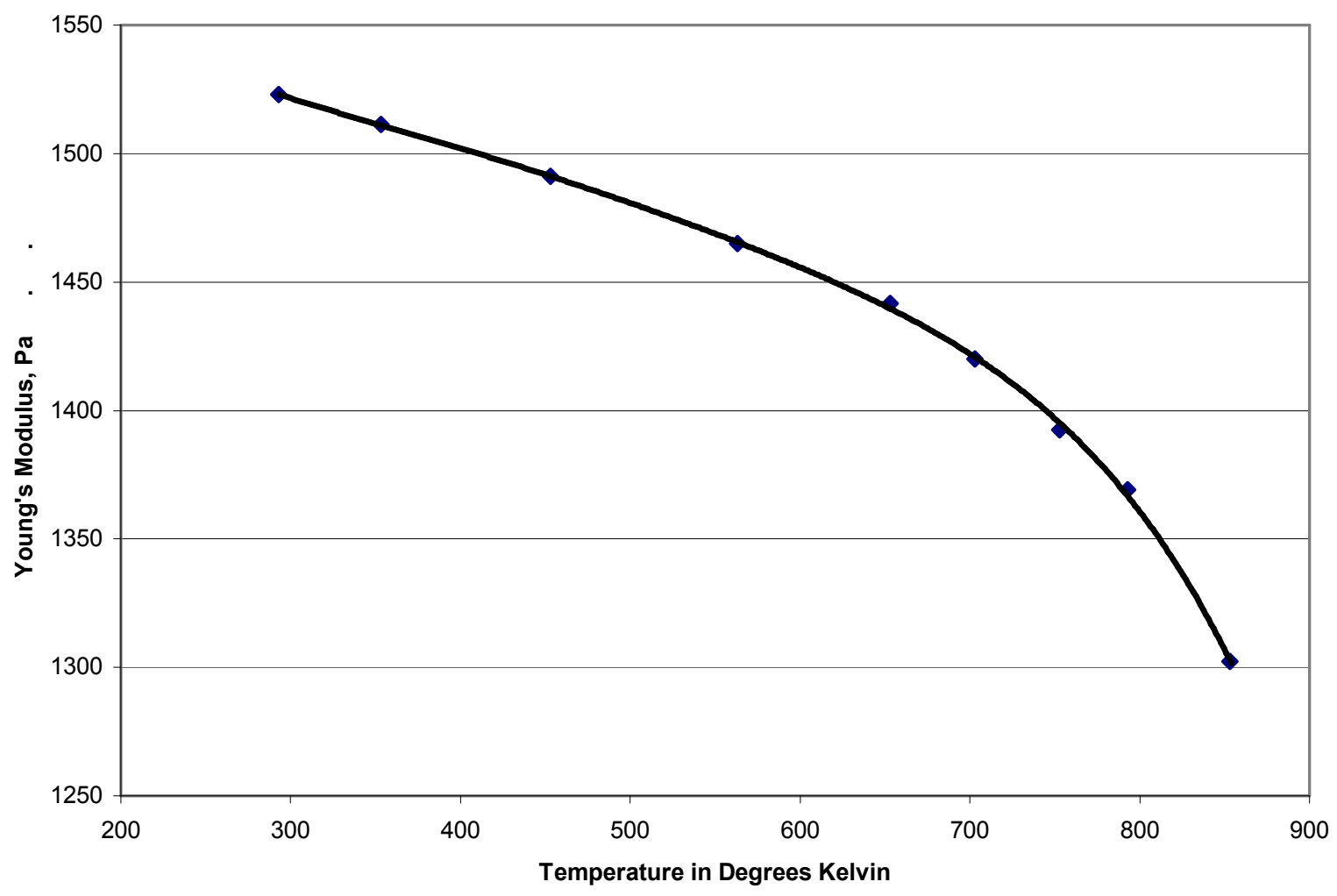

Figure 2.3.1 Young's Modulus as a Function of Temperature 
Again, this graph was derived from a graph in "Handbook of Glass Manufacture" by Fay V. Tooley. Data points were taken from the original source and entered in a spreadsheet program to derive curve fit equations not given in the original source.

As can be seen in the graph, as temperature increases, E decreases. The decrease increases in magnitude as the temperature rises. The last value on the graph is located at a temperature slightly higher than the temperature at which glass reaches the working point. At this point viscosity has become a more significant factor in the shape of the fiberglass, and the strand will be treated as a liquid by the model.

When a uniaxial stress causes elongation parallel to the stress, it also causes a contraction in the direction perpendicular to the stress. The Poisson's Ratio, $v$, is a material constant that relates the strain in the x-direction to the contraction in both the y and z-directions. Glass is an isotropic material, hence the Poisson's Ratio for both the y and z-directions are equal. According to data found in "Handbook of Glass Manufacture" by Fay V. Tooley, the value for the Poisson's Ratio of soda-lime silica glass is 0.22 . 


\subsection{Viscoelastic Theory}

During the transition from a liquid to a solid, glass displays characteristics that are a combination of liquid and solid properties. Using the data obtained from Tooley we can conclude that glass is in this phase between approximately 510 and $853 \mathrm{~K}$. Materials that behave such as glass are known as viscoelastic elements. Researchers model viscoelastic elements using combinations of elastic elements, represented as springs, and viscous elements, represented as dashpots, in various combinations of series and parallel arrangements.

The two simplest models of viscoelastic elements are the Kelvin Model and the Maxwell Model. The Maxwell model consists of a spring and a dashpot in series, whereas the Kelvin Model uses a spring and dashpot in parallel. Several sources explain these models, however, the following description is similar to what can be found in Shames and Cozzarelli's book [5].

The Maxwell model consists of a spring in series with a dashpot. Since the two elements are in series, it is true that the stress in the spring is equal to the stress in the dashpot, and the total strain is equal to the strain of the spring plus the strain of the dashpot. The characteristic equation for the Maxwell model becomes [5]:

$$
\dot{\varepsilon}=\frac{\dot{\tau}}{E}+\frac{\tau}{\eta}
$$

A paraphrased version of Shames and Cozzarelli's [5] derivation of the characteristic equations can be found in Appendix 3.

The Kelvin model combines the spring and dashpot in parallel and the governing equation can be found in a similar fashion to the Maxwell model except now the strain in the spring element is equal to the strain in the dashpot, and the total stress is the sum of the stresses in the spring and dashpot. The behavior equation for the Kelvin model becomes [5]: 


$$
\tau=\eta \dot{\varepsilon}+E \varepsilon
$$

To better understand the behavior of these two viscoelastic models, the creep test and the relaxation test can be applied. The creep test applies constant stress to the system and uses the behavior equation to determine the strain on the model as a function of time. Likewise the relaxation test applies a constant strain on the model to determine the stress in the system as a function of time.

When performing the creep test, constant stress is placed on the model to examine the strain as a function of time, therefore:

$$
\tau=\tau_{0}[\mathrm{u}(\mathrm{t})], \text { since } \mathrm{t}_{0}=0 \text { in this case, i.e. } \tau=\tau_{0} \forall \mathrm{t}>0
$$

Creep test results for the Maxwell model follow the following equation [5]:

$$
\varepsilon(t)=\tau_{0}\left(\frac{1}{E}+\frac{t}{\eta}\right)[u(t)]
$$
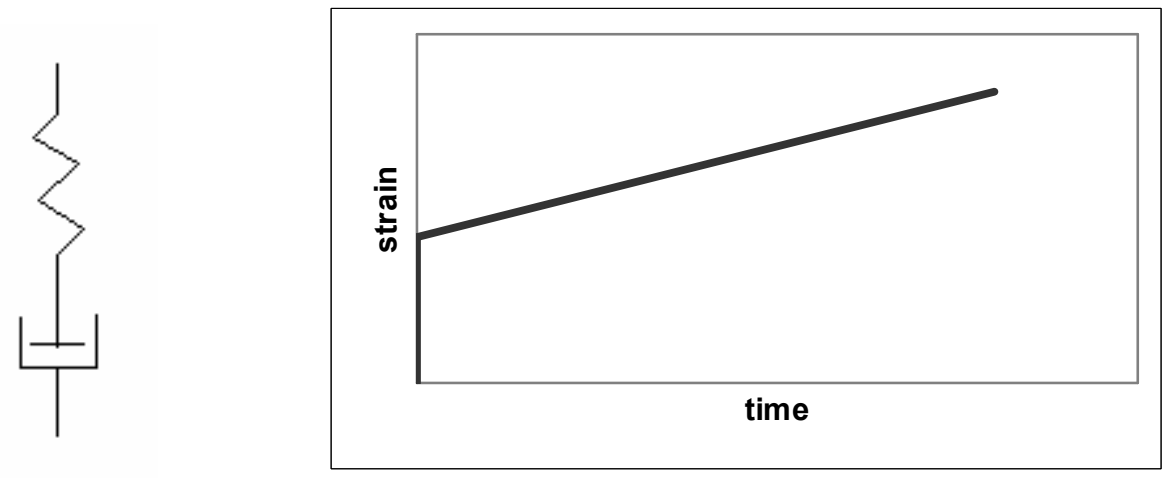

Figure 2.4.1 Maxwell Model and its Creep Test Response

Like the creep test, the relaxation tests uses the behavior equation except this time strain is kept constant.

$$
\varepsilon=\varepsilon_{0}[\mathrm{u}(\mathrm{t})]
$$

Hence the relaxation equation becomes 


$$
\tau(t)=\varepsilon_{0} E e^{-\left(E / \eta^{\prime) t}\right.}[u(t)]
$$

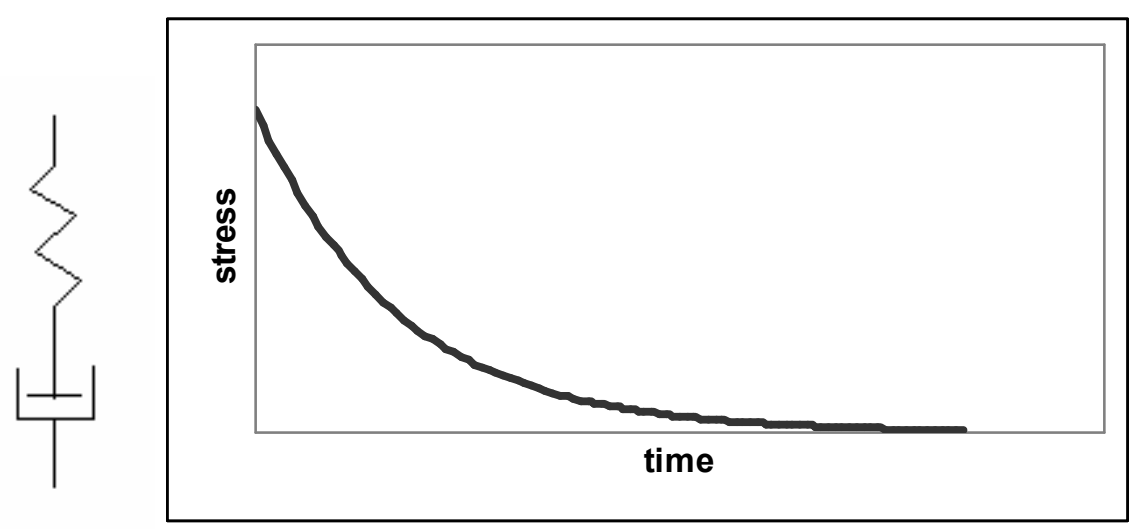

Figure 2.4.2 Maxwell Representation and Relaxation Test Results

As can be seen, a Maxwell material can be stretched to a certain length and if that length is held constant over a certain period of time the stress on the material returns to zero and that material takes the current shape until a new stress and strain is applied. This becomes an important concept later.

The Maxwell model is most commonly used to describe materials that undergo creep. Creep occurs when a material under stress reacts initially to the stress and becomes more deformed gradually over time. Engineers, for example, inspect bridges for creep to determine if the bridges need to be rebuilt due to the increased deformation. In the glass world however, the Maxwell model is used for its relaxation ability. Once glass is deformed the stress in the glass slowly returns to zero. This return to an unstressed state is much more rapid in hotter, less viscous glass.

The Kelvin model behaves very differently than the Maxwell model. Here the elastic element and viscous element are in parallel, and the behavior equation is

$$
\tau=\eta \dot{\varepsilon}+E \varepsilon
$$


Applying the creep test on the Kelvin model gives [5]:

$$
\varepsilon(t)=\frac{\tau_{0}}{E}\left(1-e^{-\left(E / \eta^{t} t\right.}\right)[u(t)]
$$
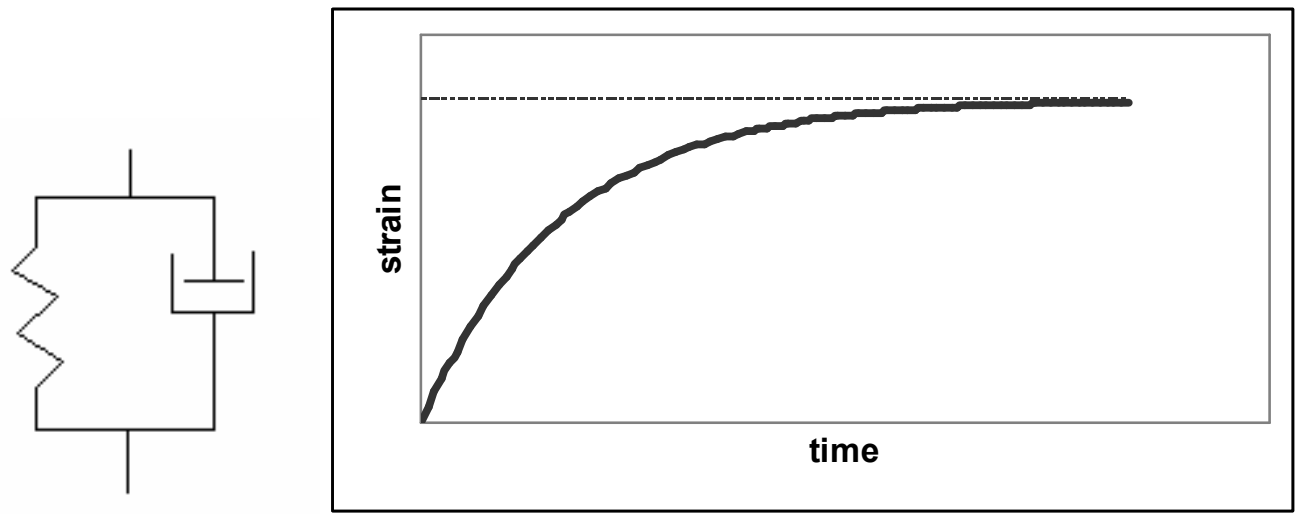

Figure 2.4.3 Kelvin Representation and Creep Test Response

Applying the relaxation test to the Kelvin model shows that theoretically the Kelvin model never relaxes.

$$
\tau(t)=\varepsilon_{0}\{\eta[\delta(t)]+E[u(t)]\}
$$

Because glass does relax under constant strain (faster at high temperatures), the Kelvin model cannot be the most accurate model for the behavior of glass; however, the Kelvin model is used very often in the glass industry because glass behaves similar to the results of the creep test. More simple glass working processes can use the Kelvin model as the primary model of behavior because they are only concerned with a deformation under one set of conditions, followed by cooling until the glass reaches is solid phase. The Kelvin model does not incorporate what happens to glass under prolonged stress. 
Several sources, including Varshneya pages 252-254, concur that the glass industry uses different models to determine the behavior of viscoelastic substances in individual processes. One of the more popular models is known as a Burger's Model, which is a Kelvin model in series with a Maxwell model. Another simple model is the three-parameter fluid model, which combines the Kelvin model with a viscous element so that the Kelvin model also shows signs of the phenomenon known as creep. Behavior equations for the three-parameter are used in the model and will be derived and analyzed in the technical approach section. 


\subsection{Heat Transfer}

One of the objectives of this thesis is to create a temperature profile of the strand along its axis from the time the glass exits the furnace until it is incorporated into the mat. By using critical parameters such as ambient air temperature inside the plant, heat transfer calculations help to create this temperature profile. Altering parameters could control what is physically happening to the glass at certain times and locations. By determining physical restraints on the glass, such as ultimate stress, the parameter changes would result in less downtime.

For the model we can assume the control volume will be a cylindrical in shape. Therefore, all theoretical concepts and formulas concerning heat transfer in this thesis can be applied to cylinders.

Convection is the heat transfer from a material to a moving fluid. The rate of heat transfer caused by convection is determined by several different characteristics of the problem. The shape of the material, the physical properties of the moving fluid, and how fast the fluid is moving all affect the heat transfer rate.

For analytical purposes the shape of the glass strand will be assumed to be a cylinder. The air flowing past the cylinder is a result of the traversing furnace. Because the strands are positioned perpendicular to the direction of movement, the fluid motion is also perpendicular to the axis of the strands.

According to Mills [4] the rate of heat transfer through convection is described as:

$$
q_{c}=h A\left(T_{w}-T_{\infty}\right)
$$


Here $\mathrm{h}$ is the heat transfer coefficient in terms of $\mathrm{E} / \mathrm{tL}^{2} \mathrm{~T}$. The $\mathrm{A}$ in this case is area perpendicular to the direction of heat flow.

Convective heat transfer is heavily dependent on the Reynolds Number. The equation for the Reynolds number for air flowing perpendicular to a cylinder is [4]:

$$
\mathrm{Re}=\mathrm{VD} / \mathrm{v}
$$

Where $\mathrm{V}$ is the velocity of the air flowing across the cylinder, $\mathrm{D}$ is the diameter of the cylinder, and $v$ is the kinematic viscosity of air.

The kinematic viscosity of air was determined in a similar fashion to the other properties discussed in this thesis previously. Mills's book [4] contained a table of points that were entered into a spreadsheet. Values can be interpolated to determine the kinematic viscosity of air at all temperatures in the model.

\begin{tabular}{l|l} 
Temp, K & $V$ \\
\hline 150 & 4.52 \\
200 & 7.69 \\
250 & 11.42 \\
260 & 12.23 \\
270 & 13.06 \\
280 & 13.91 \\
290 & 14.77 \\
300 & 15.66 \\
310 & 16.54 \\
320 & 17.44 \\
330 & 18.37 \\
340 & 19.32 \\
350 & 20.3
\end{tabular}

\begin{tabular}{l|l} 
Temp, K & $v$ \\
\hline 360 & 31.3 \\
370 & 22.32 \\
380 & 23.36 \\
390 & 24.42 \\
400 & 25.5 \\
500 & 37.3 \\
600 & 50.5 \\
700 & 65.15 \\
800 & 81.2 \\
900 & 98.6 \\
1000 & 117.3 \\
1500 & 229 \\
2000 & 368
\end{tabular}

Table 2.5.1 Kinematic Viscosity of Air, reproduced from Mills, p. 920 [4]

Another dimensionless number associated with heat transfer is the Prandtl Number. The Prandtl Number uses heat transfer constants for the fluid involved in convective heat transfer. The Prandtl Number is written as [4]: 


$$
\operatorname{Pr}=\frac{c_{p} \mu}{k}=\frac{v}{k / \rho c_{p}}
$$

The Prandtl Number for glass stays relatively constant with relation to temperature. According to Mills [4], for temperatures less than $700 \mathrm{~K}$, the Prandtl Number for glass is 0.7 . For temperatures less than $700 \mathrm{~K}$ the Prandtl Number becomes 0.69 .

A final dimensionless number is needed to relate the Reynolds Number and the Prandtl Number to the heat transfer coefficient, the ultimate goal of the analysis. This number is known as the Nusselt Number. The Nusselt Number is dependent upon the shape of the control volume and the manner at which the fluid is interacting with the solid. Because of the Nusselt Number's dependence on the fluid's interaction, it is related to the Reynolds Numbers, which from before determines the turbulence of the flow. In the case of fiber glass manufacturing the diameter of the glass strand is small enough to maintain a Reynolds Number below $10^{4}$, therefore according to Mills [4] for the flow across a cylinder with a Reynolds Number less than $10^{4}$ the Nusselt Number becomes [4]:

$$
\overline{N u}_{D}=0.3+\frac{0.62 \operatorname{Re}_{D}^{1 / 2} \operatorname{Pr}^{1 / 3}}{\left[1+(0.4 / \operatorname{Pr})^{2 / 3}\right]^{1 / 4}}
$$

Finally a determination of the heat transfer can be made using the following equation obtained from Mills [4]:

$$
\bar{h}_{c}=\left(\frac{k}{D}\right) \overline{N u}_{D}
$$

Here $\mathrm{k}$ is the thermal conductivity of the glass, which is $0.88 \mathrm{~W} / \mathrm{m} \mathrm{K}$ [4], and D is the diameter of the cylinder in meters. 
Radiation is the heat transfer through electromagnetic waves or photons. When this occurs, the rate of heat transfer is reliant on physical properties of the glass, particularly its emissivity. The equation for heat transfer due to radiation as [4]:

$$
q_{r}=\sigma \varepsilon A F_{12}\left(T_{w}^{4}-T_{\infty}^{4}\right)
$$

where $\sigma$ is the Stefan-Boltzmann constant which is approximately $5.67 \times 10^{-8} \mathrm{~W} / \mathrm{m}^{2} \mathrm{~K}^{4}, \varepsilon$ is the emissivity of glass which is approximately $0.9, \mathrm{~A}$ is the surface area undergoing radiation, $\mathrm{F}_{12}$ is the shape factor, $T_{w}$ is the temperature of surface, and $T_{\infty}$ is the ambient temperature of the air. When dealing with two finite surfaces only a fraction of the radiation from one surface reaches the second. It is because of this, shape factors are used in the heat transfer equation to more accurately determine the amount of heat transferred from one body to the other. "The shape factor is a geometrical concept and depends only on the size, shape, and orientation of the surfaces." (Mills, p. 457) [4] Development of the shape factor will be discussed in a later section. 


\subsection{Residual Stress Caused by Cooling}

Any time glass is being used in a manufacturing process where the glass is heated and cooled, there exists the possibility of residual stresses being found in the glass. This is due to uneven cooling of glass and its temperature dependent properties. Assuming all heat transfer process around the strand are symmetrical, glass on the outer edge of the product cools faster due to convection and radiation both acting on the surface, therefore creating a parabolic temperature distribution inside the glass. This in turn causes parabolic stress distributions across the crosssection of the glass. Glass on the outside would experience constant tension, while glass on the inside experienced compression. Varshneya pages 297-298 [3] claims that if the temperature distribution on the cross-section remained constant throughout the cooling process it would cause the stresses to disappear, because the strand experiences strain and relaxation

Fiberglass is a special case. Because of the small diameters of the fibers, the ratio of volume to surface area is small. This means the temperature across the strand is nearly uniform. Confirmation of this can be found in what is known as the Biot number.

The Biot number is a ratio comparing the internal thermal resistance to the thermal resistance at the surface. According to A.F. Mills page 32 [4], the Biot number can be represented mathematically with the following equations:

$$
\frac{\text { Internal conduction resis } \tan c e}{\text { External convection resis } \tan c e} \approx \frac{L / k_{s} A}{1 / h_{c} A}=\frac{\overline{h_{c}} L}{k_{s}}
$$

where $\mathrm{k}_{\mathrm{s}}$ is the thermal conductivity of the solid material. This equation pertains to heat transfer through a flat plate of area $=\mathrm{A}$ and thickness equal $\mathrm{L}$. This equation also does not make use of 
the thermal resistance associated with radiation. In order to use the lump sum method on a cylinder, $\mathrm{L}$ must be replaced with $\mathrm{r} / 2$, where $\mathrm{r}$ is the radius of the cylinder.

The final value for the Biot number is dimensionless. Mills page 32 [4] states that bodies having a Biot number less than 0.1 ensures that internal temperatures will vary less than $5 \%$ of surface temperatures on the body. This justifies an assumption that bodies having $\mathrm{Bi}<0.1$, have a uniform temperature throughout. 


\subsection{Surface Tension}

Liquids are unable to expand freely like gases and therefore create an interface with another liquid or gas. Molecules on the inside of the mass of liquid try to expand while molecules on the interface are attracting to each other. This creates tension on the surface.

Figure 2.7.1 shows a liquid whose shape is in the form of a cylinder, which is the approximated shape of a section of fiberglass. An arbitrary line is drawn on the surface of the cylinder parallel to its axis. Here, tension can be illustrated acting along this line the entire length of the cylinder.

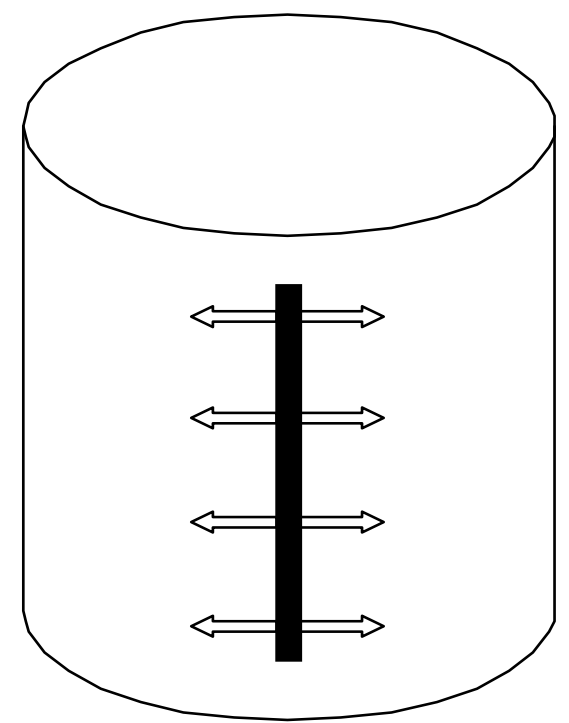

Figure 2.7.1 Illustration of Surface Tension on a Cylinder

According to White (p. 29) [11], the pressure increase in the interior of a liquid cylinder is balanced by two surface-tension forces

$$
2 R L \Delta p=2 \mathrm{Y} L
$$

which reduces to

$$
\Delta p=\frac{\mathrm{Y}}{R}
$$


Here $\mathrm{R}$ equals the radius of the cylinder, $\mathrm{L}$ is the length of the cylinder, $\mathrm{Y}$ is the surface tension, and $\mathrm{p}$ is pressure. The pressure on the surface will be considered as an extra stress on the strand. The force caused by surface tension in the axial direction will also be addressed by the model. 


\subsection{Nozzle Design}

An important element of the fiberglass spinning process is the nozzle. Nozzles can be designed using a wide range of materials, sizes, and shapes. According to Loewentstein [12], nozzle design is important because the rate of fiber manufacture is entirely dependent on the flow through the nozzle. The flow rate through the nozzle depends on the radius and length of the cylindrical portion of the nozzle, the viscosity of the glass in the furnace and the height of the glass in the furnace. Flow rate is independent of the attenuation speed. Therefore running the drums at higher speeds only decreases the strand diameter. If other variables specific to the nozzle flow rate remain unchanged, productivity, as far as total weight is concerned, is not increased. Ultimately smaller strands are more valuable than larger strands as they tend to increase the functionality of the final product. From Loewentstein (page 90) [12], the equation governing the flow of molten glass through a nozzle can be described in relation to Poiseuille's equation as:

$$
F \propto \frac{r^{4} h}{l \eta}
$$

where $\mathrm{F}$ is the rate of flow, $\mathrm{r}$ is the radius of the nozzle bore in its narrowest cylindrical section, 1 is the length of this cylindrical section, $h$ is the height of glass above the nozzle, and $\eta$ is the viscosity of the glass in the nozzle. 


\subsection{Existing Control Ideas}

Several methods have been used in the past for temperature control in the fiberglass industry. One method mentioned in an article from FluentNEWS [10] is a quench box. Here the glass is extruded through the nozzles beneath the furnace and pulled through a box. This box uses flowing gases with various heat transfer properties to generate a higher, controllable, convective heat transfer rate. A module developed by Fluent, Inc. boasts the ability to balance heat, mass, and momentum between fibers and the surrounding gas.

The subject plant, however, uses a different method for controlling the temperature of the glass. Instead of a specialized gas, air is used as the medium for cooling. Beneath the furnace is a hood that decreases the amount of airflow across the strands making radiation a more dominant form of heat transfer. Inside the hood coils are used to give the strands a body to which they can radiate heat. Specific details of the system will not be included in this text to insure the privacy of the subject company.

The number of coils present and the fluid within the coils plays an important role in the cooling of the glass. Because heat transfer rate is dependent on the temperature difference between the object and the medium, a lower ambient temperature would mean higher heat transfer rates due to radiation and convection and maintaining a low fluid temperature inside the coils would mean higher heat transfer rates due to radiation.

The second page of a journal article by Christopher Jian, Ph.D. [8] describes a problem often faced in the fiberglass industry. Dr. Jian states that one challenge in the process is making sure tiny gas bubbles trapped in the molten glass are eliminated prior to forming. If the viscosity

of the glass is too high (result of lower temperatures) bubbles are unable to rise to the surface of 
the molten glass and escape. If a bubble were to exit via a nozzle, breakage would be inevitable. This puts a limit on furnace temperature adjustment in the overall control of the system.

Loewenstein relates the probability of breakage to the attenuation rate, claiming that the stability at the base of the meniscus decreases with increasing rates of attenuation in excess of $2500 \mathrm{~m} / \mathrm{min}$. Loewenstein goes on to say that this increased probability does not increase that rapidly so it is possible for plants to perform at higher attenuation rates $(3000-4000 \mathrm{~m} / \mathrm{min})$ and balance breakage with economics. The attenuation rate at the subject plant is approximately 900 $\mathrm{m} / \mathrm{min}$. This is well below the safe limit. 


\section{Technical Approach}

All the concepts discussed in the Literature Review section will be used to develop a working model of the spinning process. The model will be developed in the program MATLAB. The final model of the glass drawing process will use a position marching process to determine physical aspects of a single strand of glass during steady state at different distances from the furnace, particularly strand diameter and temperature. Using a position marching process allows for temperature dependent properties to be updated regularly along the strand. The position steps will begin where the glass exits the die and end after the strand has reached final diameter.

The model is divided into three sections based on the three phases glass undergoes during the cooling process: fluid, transition, and solid. Before it is possible to model these sections mathematically, basic mathematic principles must be applied. Physical properties will first be analyzed followed by heat transfer concepts.

The first step of the code is assigning values to physical constants, and design variables. Vectors are initialized for the distance from the plate, the diameter of the strand, and the temperature. The initial condition for the distance from the plate is zero meters. The initial diameter is equal to the diameter of the nozzle, in this case $4.27 \mathrm{~mm}$. The initial temperature is the temperature of the glass inside the furnace. This is a variable parameter, but when generating a standard model the value will be set for $1384 \mathrm{~K}$, an actual measurement taken at the subject plant.

The initial temperature is then plugged into the best-fit equations for the different temperature dependent variables. These become the first set of values used in the calculations performed by the model. 


\subsection{Concept of Model Generation}

The first step in the model generation is assigning values to certain working conditions and desired results, such as drum speed and final strand diameter respectively. Because the workers can safely handle the strands as they place the strands on the drum, it can be assumed that the temperature of the strand when it reaches the drum is approximately the ambient temperature of the room.

Drum speed plays a major role in the final product. The tensile force acting on the glass strand is a function of drum speed. It can also be used to establish a mass flow rate based on the final diameter of the strands and the density of the glass at the final temperature. Due to conservation of mass principles the mass flow rate will be constant throughout the entire strand. The following formula can be used to determine the mass flow rate of the system.

$$
\dot{m}=\rho A V=\rho \frac{\pi}{4} d^{2} V
$$

Here, $\rho$ is the density of glass at ambient temperature, $d$ is the final diameter of the strand (to establish the flow rate for the current nozzle this diameter will be estimated at 28.5 microns), and $\mathrm{V}$ is the tangential velocity of the drum at the point of contact with the glass. In this model $\mathrm{V}$ is equal to $18 \mathrm{ft}$ times the angular velocity in rpm. A drum speed of $149 \mathrm{rpm}$ establishes a final diameter of 28.5 microns using the current system settings.

It is now possible to start the proposed model by entering values at the base of the nozzle where $\mathrm{X}$, the distance from the die, equals zero, the strand diameter equals the diameter of the nozzle, and the temperature equals the recorded temperature of the glass. Using the property graphs, the values for viscosity, modulus of elasticity, and density can be obtained. The velocity of the glass at this point can be obtained using the mass flow rate. 


$$
V_{i}=\frac{\dot{m}}{\rho_{i} \frac{\pi}{4} d_{i}^{2}}
$$

These become the initial conditions for the model.

The theory behind the model is that it is possible to superpose flow rate and strain on the material in sections where strain, held at a constant stress, goes to infinity. In other words, the model supposes that as glass flows at a certain rate it is also pulled and stretched at another rate and these two increases in length can be added together to determine a new length and diameter. This theory will be tested after the creation of the model by comparing data from the model to actual data from the plant.

The model uses a step method to determine the shape of the fiberglass. The first step occurs at the nozzle. Using the obtained velocity equation (3.1.2), a time can be determined for a theoretical cylinder, with a diameter equal to the current step's strand diameter, to be formed with length equal to $\Delta \mathrm{X}$. The model uses $\Delta \mathrm{X}=0.001$ meters. This creates a new control volume fixed in space at every step. This same theoretical volume is being deformed by the tension in the strand over the same time period. Using the time it takes to create the cylinder in the behavior equation established for the model at this step, it is possible to obtain a new deformed cylinder. The length of this new cylinder is added to the current step's X coordinate, and the new diameter is recorded.

This concept can better be illustrated with Figure 3.1.1. This figure shows the first control volume directly below the nozzle. The black rectangle represents the theoretical cylinder formed by the flowing glass at the beginning of the current step's calculations. The red rectangle is used to illustrate the theoretical cylinder (the black cylinder) after deformation. Finally the 
blue line connects the points determined by the $\mathrm{X}$ and $\mathrm{D}$ vectors illustrating the theoretical shape of the control volume fixed in space.

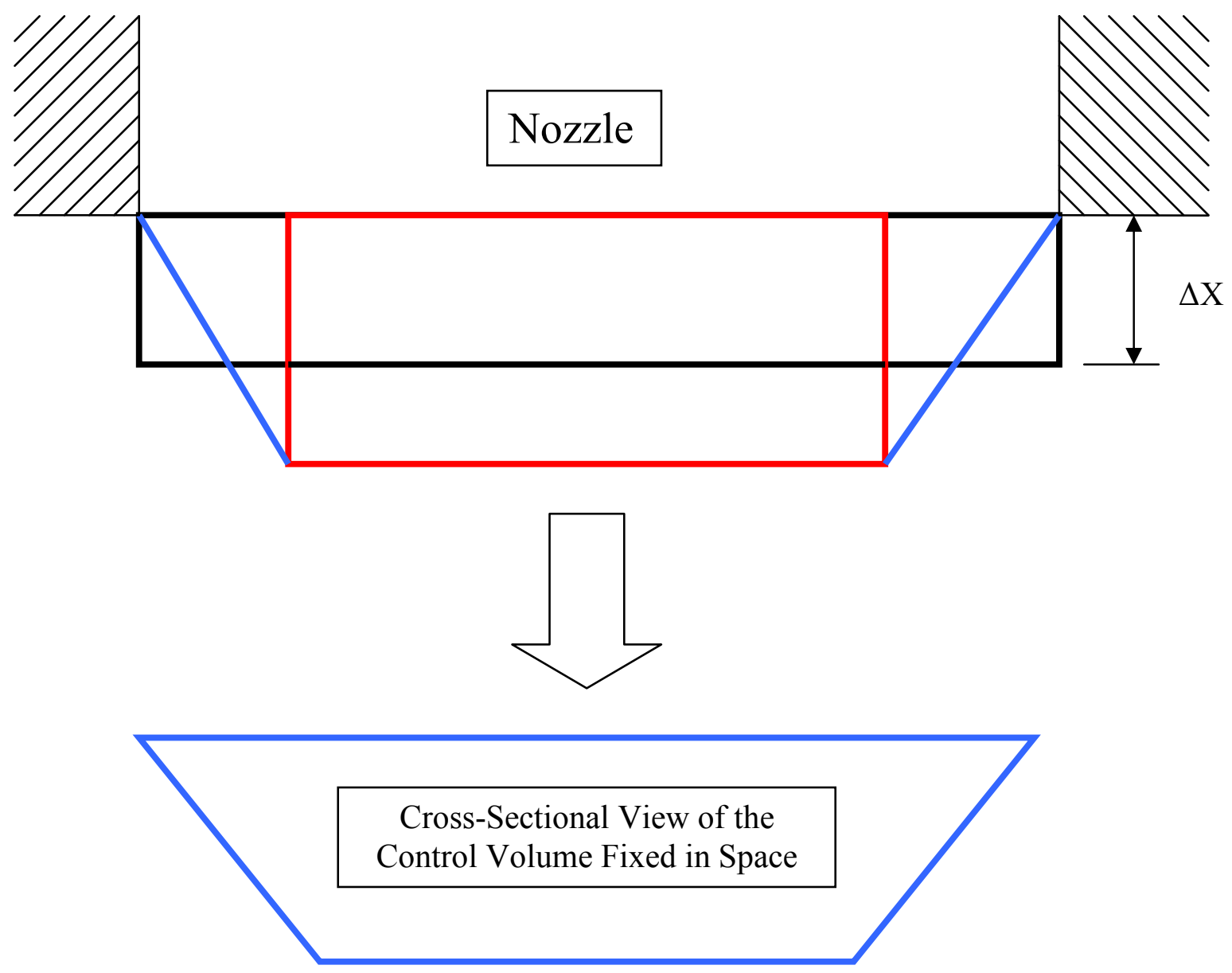

Figure 3.1.1: Illustration of the first control volume fixed in space

In this particular situation, the $\mathrm{X}$ coordinate equals zero and the Diameter equals 0.00427 meters at the nozzle. If the mass flow rate determines the velocity of the glass at that diameter and density to be $1 \mathrm{~m} / \mathrm{s}$, then the time it would take to create a theoretical cylinder would be 0.001 seconds. If the behavior equation determines after 0.001 seconds, the $4.27 \mathrm{~mm}$ diameter cylinder with a length equal to $0.001 \mathrm{~m}$ is strained to 0.0012 meters in length with a new 
diameter of $0.00417 \mathrm{~m}$, these numbers determine the next values in the distance and diameter vectors, hence the vector, $X$, becomes [0 0.0012] and $\mathrm{D}=[0.004270 .00417]$.

Using heat transfer theory to obtain a new temperature, all of the values are updated at this new X coordinate and diameter and the same procedure is applied again. Instead of using the same control volume of incompressible liquid throughout the calculations, a new control volume is generated at the beginning of each step. The new theoretical cylinder of length $\Delta \mathrm{X}$ will have a diameter equal to 4.17 millimeters (using the example given above). Soon there will be a series of $\mathrm{X}$ coordinates and their corresponding diameters and temperatures. The diameter and temperature of the strand as a function of distance, $\mathrm{X}$, can later be plotted and connected with a smooth line giving a profile of the glass strand.

Figure 3.1.2 illustrates the creation of the sequential steps after the first step below the nozzle. The color scheme from Figure 3.1.1 continues in the following figure. 


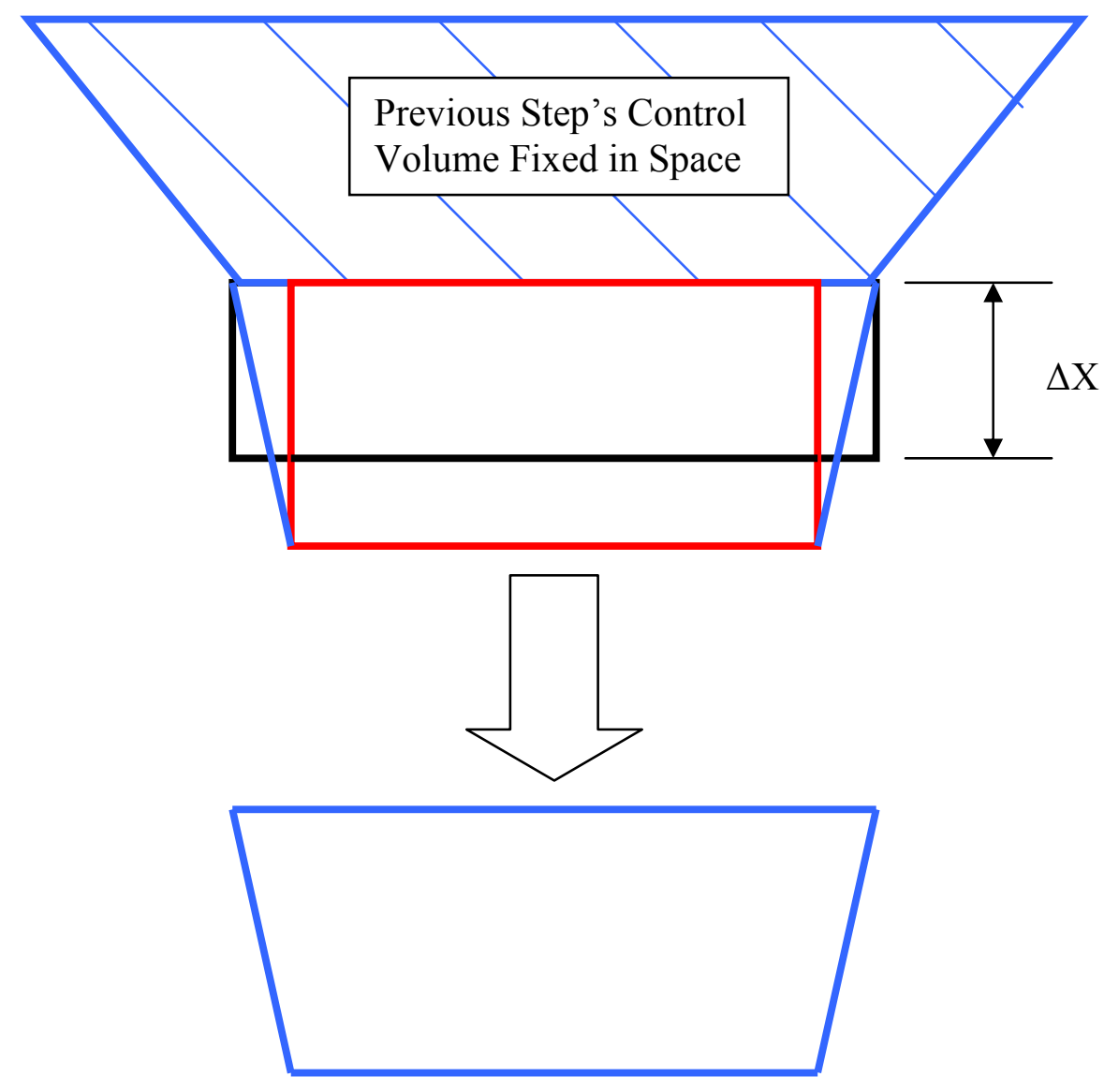

Cross-Sectional View of the NEW Control Volume Fixed in Space

Figure 3.1.2 Illustration of Continual Control Volume Generation

Finally figure 3.1.3 shows the entire sequence of control volumes to illustrate the final shape of the glass through the blue lines. Note that this image is not to scale but merely a theoretical representation of the final product. 


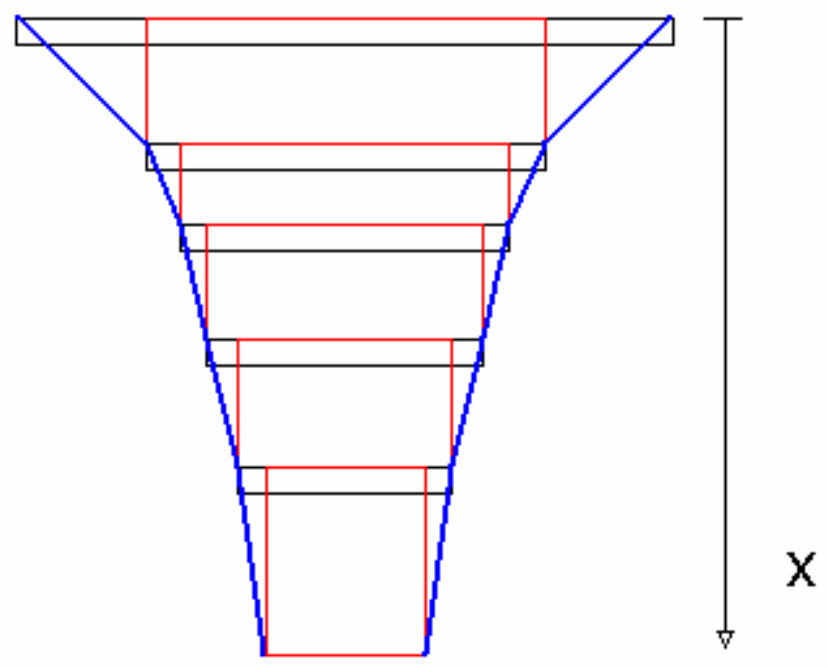

Figure 3.1.1 Illustration of Theoretical Procedure 


\subsection{Strain In The Liquid Phase}

When the temperature of glass is extremely high, it does not display any elastic properties and can therefore be assumed liquid. The liquid phase of glass is heavily dependent upon viscosity when determining how it will behave under stress. The behavior equation for strain used in the liquid phase is taken from Vershneya page 184 [3].

$$
\sigma_{x}=\lambda \dot{e}_{x}
$$

Here $\sigma_{\mathrm{x}}$ is the stress on the glass, $\lambda$ is the coefficient of viscous traction or 3 times the viscosity, and $\mathrm{e}_{\mathrm{x}}$ is the strain. This equation is identical to the equation representing a single dashpot and will be treated as such.

When a viscous element is put under constant stress, the strain on that element goes to infinity linearly with time. The viscosity of the substance determines the slope of the line. Because the viscosity of glass changes with temperature, so does the rate at which strain approaches infinity.

Figure 3.2.1 shows how this concept is applied to the step method used in the model. 


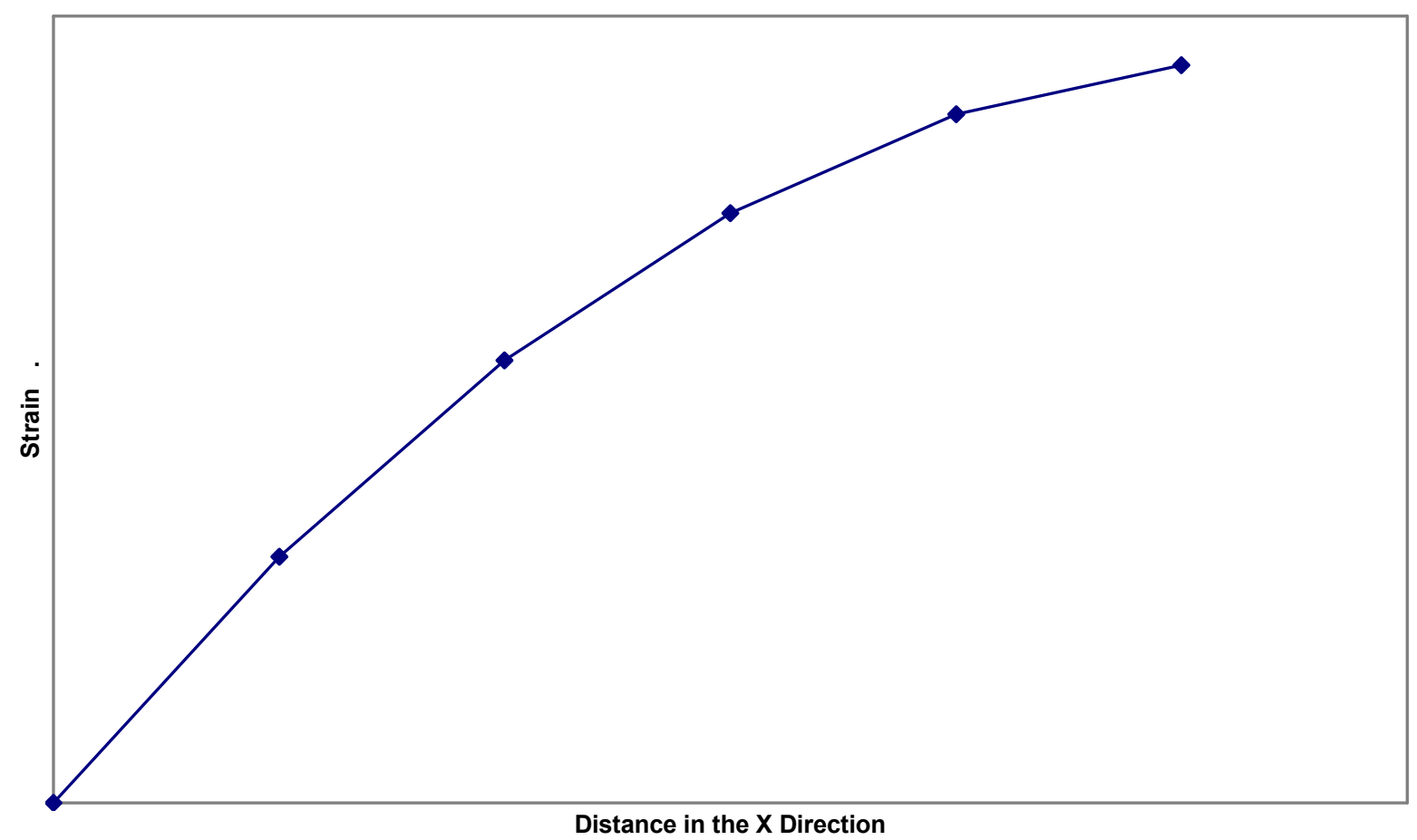

Figure 3.2.1 Strain Rate Varying with Distance

At each step the temperature of the glass decreases causing the viscosity of the glass to increase. The graph shows that at each step as the viscosity increases the strain rate decreases. The new strains are continued from the previous step. This continues until the glass reaches the transition phase. 


\subsection{Strain in the Transitional Phase}

Once the glass cools to a temperature where the Modulus of Elasticity begins to have a larger affect on the strain of the glass, the model will be replaced with a model of a viscoelastic

element. One of the biggest assumptions made in the model is the viscoelastic behavior of glass. It is true that glass does exhibit viscoelastic behavior and that the elements, elastic and viscous, are linear; however, the combination of linear elastic and viscous elements is hard to determine without experimental data to compare to theoretical data.

The viscoelastic element chosen for the model is known as the three-parameter fluid. This model has a dashpot in series with a Kelvin model, which from before is a spring element in parallel with a dashpot. It was shown that when loaded with a constant stress, a Kelvin model exhibits a slower, exponential rise to its final strain, unlike the case where a spring element is in series with a dashpot and the strain from the elastic element appeared instantaneously. Adding the second viscous element in series with the Kelvin model allows the element to display the continuing effects of stress on glass over extended periods of time.

The following derivation uses the same techniques described in Shames and Cozzarelli's book [5] for Burger's Element. In order to derive the behavior equation for the three-parameter fluid, the model must be divided into sections, denoted as 1 and 2. Section 1 will include the linear viscous element and section 2, the Kelvin model. Equations similar to the equations for a Maxwell model can now be written.

$$
\begin{aligned}
& \tau=\tau_{1}=\tau_{2} \\
& \varepsilon=\varepsilon_{1}+\varepsilon_{2} \\
& \dot{\varepsilon}=\dot{\varepsilon}_{1}+\dot{\varepsilon}_{2}
\end{aligned}
$$


Writing the behavior equation for section 1:

$$
\dot{\varepsilon}_{1}=\frac{\tau_{1}}{\eta}
$$

Because section 2 is a Kelvin element, the behavior equation is already known to be:

$$
\tau=\eta \dot{\varepsilon}+E \varepsilon
$$

Rewriting these equations in D format (replacing all d/dt with D) and solving for $\varepsilon$, the following equations can be formed:

$$
\begin{gathered}
\varepsilon_{1}=\frac{\tau_{1}}{\eta D} \\
\varepsilon_{2}=\frac{\tau_{2}}{\eta D+E}
\end{gathered}
$$

Finally these equations can be substituted into the original equations to give the behavior equation for the three-parameter fluid.

$$
\begin{gathered}
\varepsilon=\frac{2 \eta D+E}{\eta^{2} D^{2}+\eta E D} \tau \\
\eta^{2} \ddot{\varepsilon}+\eta E \dot{\varepsilon}=2 \eta \dot{\tau}+E \tau
\end{gathered}
$$

When the creep test is applied to the three-parameter fluid, a constant stress is placed on the element and the strain as a function of time can be obtained. In this particular situation it is possible for creep test results from the linear viscous element and the Kelvin model to be superposed onto on another, eliminating the need to solve the differential equations. This is because the linear viscous element is in series with the Kelvin Model, and when they are in series the stress on both elements is equal and the total strain becomes the combination of the strain on each element. The creep test results for the three-parameter fluid becomes:

$$
\varepsilon(t)=\tau_{0}\left[\frac{1}{E}\left(1-e^{-\left(E / n^{2 t}\right.}\right)+\frac{t}{\eta}\right][u(t)]
$$



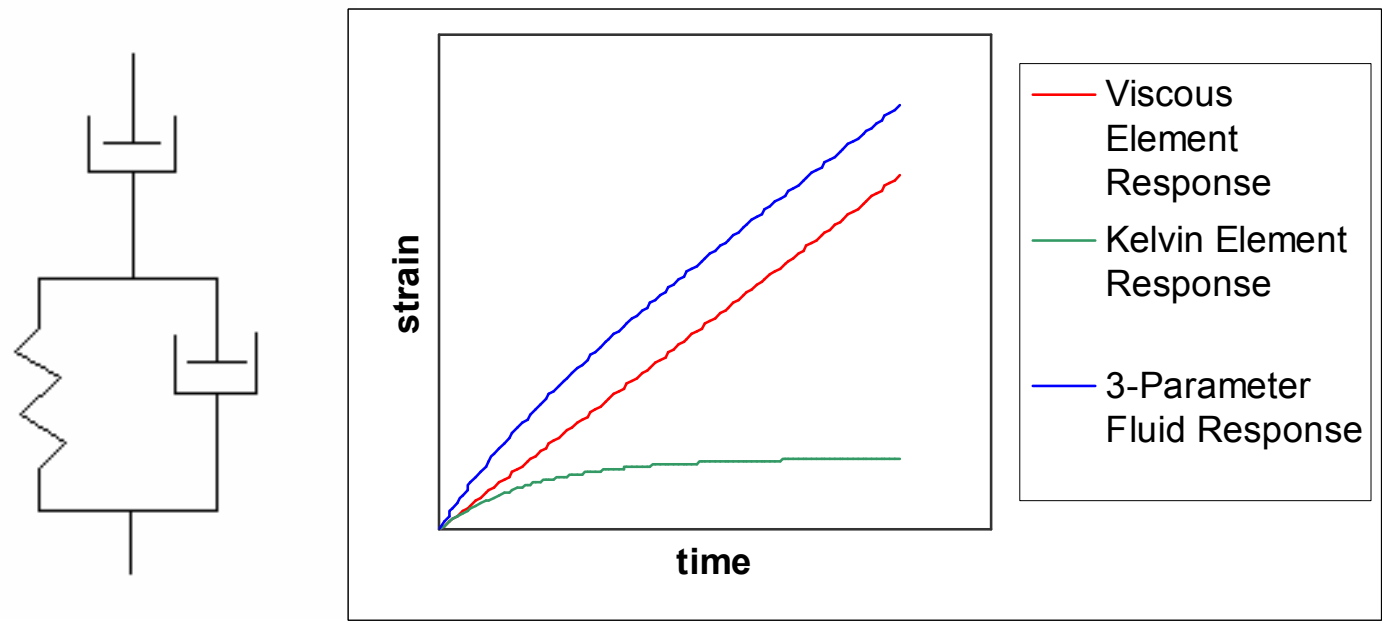

Figure 3.3.1 Three-parameter Fluid Representation and Creep Test Response

Closer inspection of equation 3.3.10 shows that, simplified, the equation for a 3-

Parameter Fluid is the sum of the Kelvin element's equation and the equation for the viscous element. The model replaces the viscous element portion of the 3-Parameter Fluid Equation with equation (3.2.1) established for the viscous element in the fluid stage. This keeps the equation more consistent.

Instead of calculating the exact relaxation curve, it is only important to show that the stress in the glass will return to zero if given a long enough time. This can be shown using LaPlace transforms and limit theorems. Taking the LaPlace transform of the behavior equation and solving for $\tau$ gives the following equation. Note that the LaPlace transform of $\varepsilon(\mathrm{t})$ becomes $\varepsilon_{0} / \mathrm{s}$

$$
\tau(s)=\frac{\eta^{2} s^{2}+\eta E s}{2 \eta s+E} \frac{\varepsilon_{0}}{s}
$$

The limit theorem suggests that

$$
\tau(\infty)=\lim _{s \rightarrow 0} s \tau(s)
$$


Therefore,

$$
\tau(\infty)=\lim _{s \rightarrow 0} \frac{\eta^{2} s^{2}+\eta E s}{2 \eta s+E} \varepsilon_{0}=\frac{0}{E}=0
$$

In order to perform the superposition technique applied to the liquid phase of the glass one slight difference has to occur. Because of the addition of the Kelvin element, the characteristic equation is no longer linear. It is impossible to change the values at the end of each step and restart the characteristic equation from a time of zero. Because the beginning of the characteristic curve has a much larger slope, the model would gain additional strain at the beginning of every step until the strain reached exceedingly inaccurate levels. To stop this, the code determines the strain from the previous step and finds the time it would take the new property values and equation to strain an equal amount. The control volume generation time is added to that time and a new strain is found. The difference of these two strain values becomes the strain applied to the glass at that particular step and its respective physical property values. 


\subsection{Strain in the Solid Phase}

During the final stages of the manufacturing process, when the glass is near the drum, temperatures are low enough that the glass is in its completely solid state and displays characteristics of a linear elastic solid. Linearly elastic materials display a relationship between stress and strain in a uniaxial direction. Since the process uses a spinning drum to apply force and attenuate the strand of glass only uniaxial stresses will be needed in the model. The equation used to determine the behavior of the glass in the solid stage becomes:

$$
\sigma_{\mathrm{x}}=\mathrm{E} \varepsilon_{\mathrm{x}}
$$

Here $\sigma_{\mathrm{x}}$ is the stress, $\mathrm{E}$ is Young's Modulus (obtained from the best-fit equation at that step's temperature), and $\varepsilon_{\mathrm{x}}$ is the strain.

For materials that are isotropic, such as glass, the formula relating strain in the $\mathrm{x}$-direction to strain in the $y$ and $z$-directions is:

$$
\varepsilon_{\mathrm{y}}=\varepsilon_{\mathrm{z}}=-v \varepsilon_{\mathrm{x}}
$$

The value for the Poisson's Ratio, v, for Soda-Lime Silica glass is 0.22 .

Unlike the liquid and transition phases, the strain in the solid phase does not go to infinity with time. Therefore, once the glass reaches the solid phase continuing the strain at a different rate does not apply. Instead strain after this point becomes constant until the glass is incorporated onto the mat. 


\subsection{Balancing Forces to Determine Strain}

The most important variable influencing the final diameter, and ultimately leading to the breaking of the strands is the stress on the strand. Stress is dependent on the forces acting on the strand, particularly the tension between the furnace and the drum. The tension on the strand is constant throughout the entire length of the strand. During the liquid phase of the glass surface tension can also be taken into account.

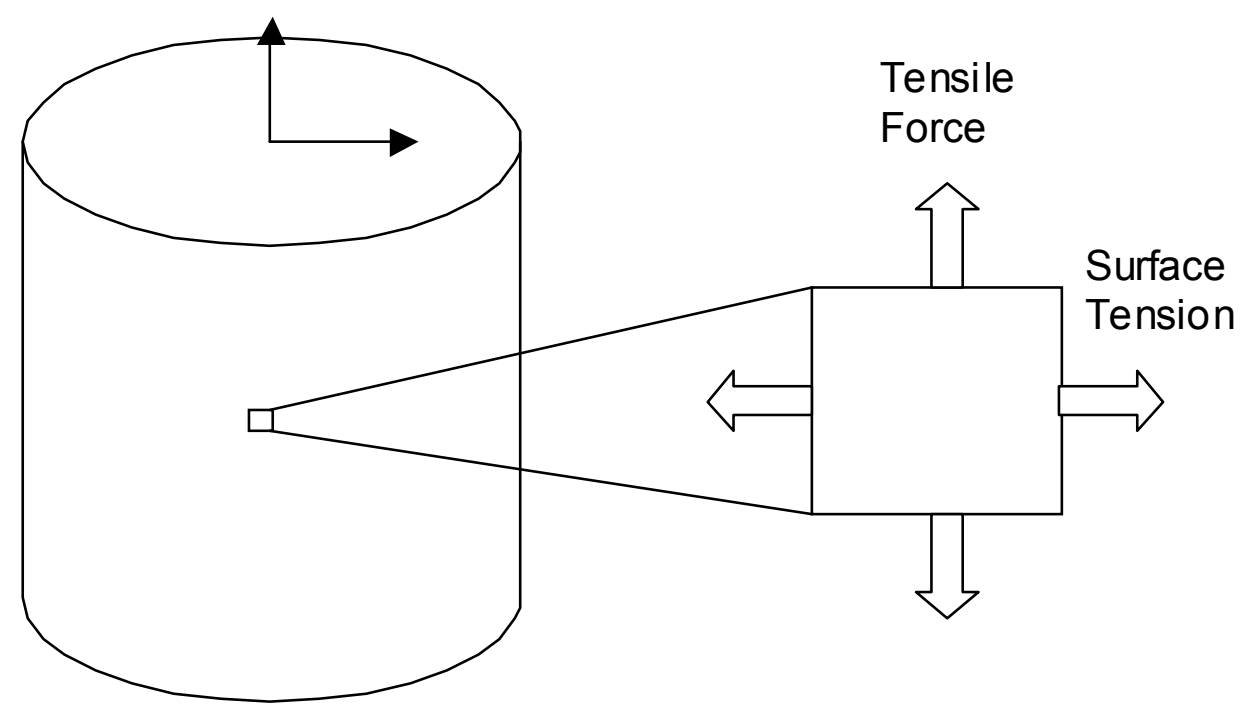

Figure 3.5.1 Force balance illustration

Figure 3.5.1 illustrates an infinitesimally small two-dimensional section on the surface of the cylinder. There are two forces acting congruently on one another: the tensile force acting in the direction of the axis, and force caused by surface tension.

In order to determine a value for tensile force, the model determines a nozzle flow rate based on the standard model. The model then uses the drum speed to determine a diameter based on the mass flow rate. From here the model works backwards to determine the tensile force needed to arrive at the final strand diameter. These forces can be recorded along with the 
different variable combinations to analyze how the force was affected. Because this tensile force is found working backwards without a direct equation, it can be assumed that all tensile forces involved are accounted for, including surface tension effects in the axial direction and the force caused by the drum.

The tensile stress can be determined using the following equation:

$$
\tau_{x}=\frac{\text { Tension }}{\text { Area }}
$$

Likewise the stress caused by the surface tension will be equal to the pressure on the surface of the cylinder:

$$
\tau_{d}=\frac{\mathrm{Y}}{R}
$$

In order to balance these two stresses and determine how each affect the strain in the liquid phase, the corresponding Poisson's ratio must be used to determine the resulting stress values.

$$
\begin{aligned}
& \tau_{x}=\tau_{x}-v \tau_{d} \\
& \tau_{d}=\tau_{d}-v \tau_{x}
\end{aligned}
$$




\subsection{Approach to Heat Transfer}

The heat transfer equations used in the final model come from principles of conservation of energy, the First Law of Thermodynamics. Here the total energy in from mass transfer, heat transfer, and work is balanced with the total energy out of the system. According to Çengel and Boles [6] the energy balance for Steady-Flow Systems becomes

$$
\dot{Q}_{\text {in }}+\dot{W}_{\text {in }}+\sum \dot{m}_{\text {in }} \theta_{\text {in }}=\dot{Q}_{\text {out }}+\dot{W}_{\text {out }}+\sum \dot{m}_{\text {out }} \theta_{\text {out }}
$$

here,

$$
\theta=C p T+\frac{V^{2}}{2}+g z
$$

Because the step sizes are so small it is safe to assume that the difference in kinetic energy and potential energy is negligible. The work terms can also be eliminated from the equation due to the fact that there is not any work acting on the system inside the control volume. These modifications to the energy balance equation transform the energy balance into the following equation [6]:

$$
\dot{Q}_{\text {in }}+\dot{m} C p_{@ T=T_{\text {in }}} T_{\text {in }}=\dot{Q}_{\text {out }}+\dot{m} C p_{@ T=T_{\text {out }}} T_{\text {out }}
$$

Due to conservation of mass the mass flow into the system equals the mass flow out of the system. 


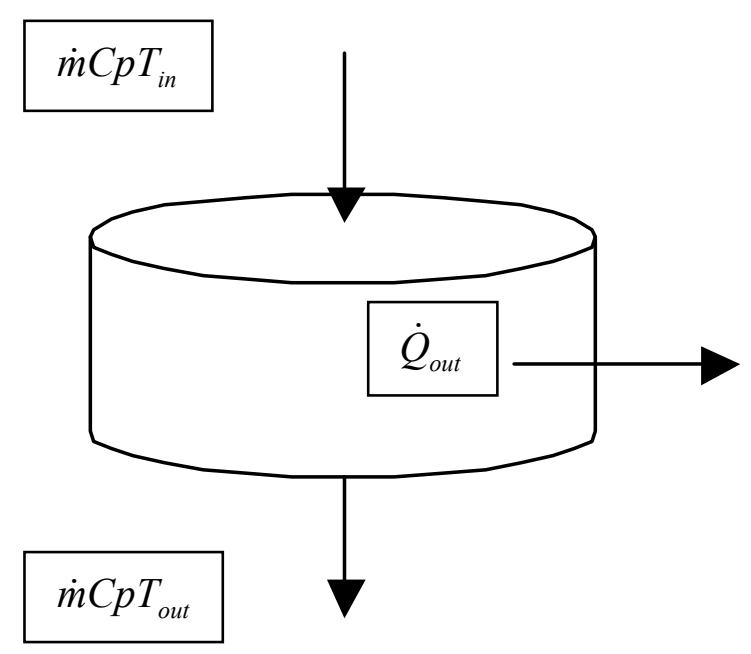

Figure 3.6.1 Illustration of Energy Flow in a Strand Section

One of the conditions acting on the surface of the control volume is Radiation. The rate of heat transferred, in this occasion, is dependent on the surface area, the emissivity of glass, the shape and position of the bodies sending and receiving the waves, and their temperature difference. The rate of heat transfer through radiation is described as [4]:

$$
q_{r}=\sigma \varepsilon A F_{12}\left(T_{w}^{4}-T_{\infty}^{4}\right)
$$

where $\sigma$ is the Stefan-Boltzmann constant which is approximately $5.67 \times 10^{-8} \mathrm{~W} / \mathrm{m}^{2} \mathrm{~K}^{4}$, and $\varepsilon$ is the emissivity of glass. $F_{12}$ is the shape factor.

The geometry and special relation between the object radiating heat and the object absorbing it determine the shape factor. In this case the objects radiating and absorbing heat are the strands and the coils, respectively. The model is based on an approximate geometry from the subject plant in order to maintain the subject company's privacy of specific details. As the glass is being drawn from the furnace, coils containing chilled water surround it. To simplify the equations, the walls of coils will be approximated as flat plates 10 centimeters wide boxing in the glass strands. The strand sections will be approximated as cylinders with a diameter equal to the 
average diameter of the cone-shaped section. From here, a geometric analysis can be made to determine the shape factor for the radiation from the glass to the coils surrounding it.

Figure 3.6.2 shows a view of the glass strand and the coils in a direction parallel to the strand. From this view it can be shown that one hundred percent of radiation from all locations on cylinder traveling away from the surface intercepts the wall of coils at some point. Therefore the shape factor is determined by the geometric relationship of the cylinder and the coils from a view perpendicular to the strand axis.

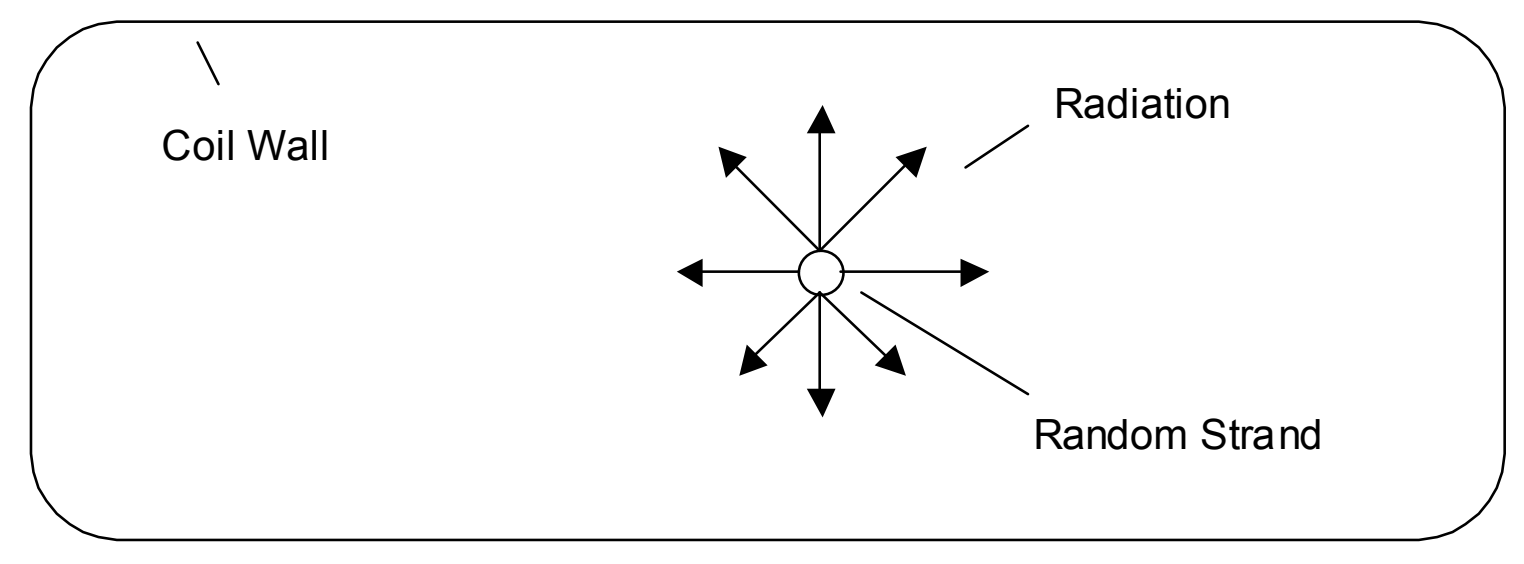

Figure 3.6.2 Illustration of Radiation Viewed Parallel to Strand Axis

Figure 3.6.3 shows the geometry of the system from a view perpendicular to the axis of the glass strand. In a two-dimensional sense, the edge of the strand can be viewed as a line with a length equal to the length of section. The surface of the coil walls will also be approximated as a flat line with a length of 10 centimeters. As can be seen one hundred percent of the radiation from this view does not come in contact with the coils. 


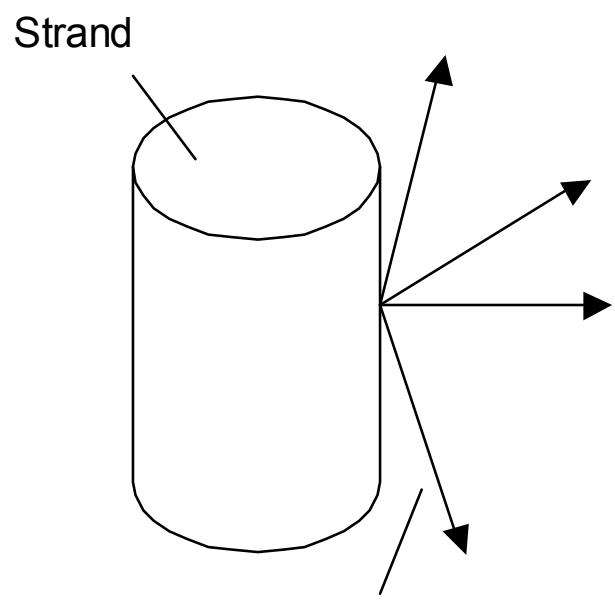

Radiation

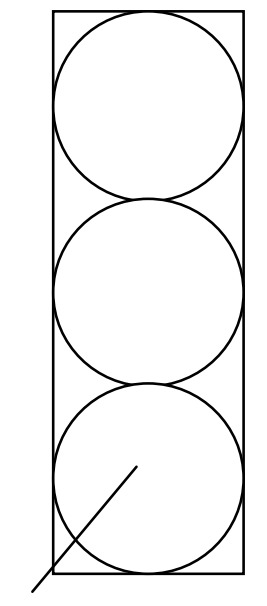

Coils (Box Approximation)

Figure 3.6.3 Radiation from a View Perpendicular to the Strand Axis

According to Mills page 464 [4], the shape factor of for this two dimensional geometry can be found using Hottel's "string rule". Figure 3.6.4 shows the two-dimensional configuration to use with the "string rule". A similar figure can be found in Mills's book [4] on page 464 .

L2

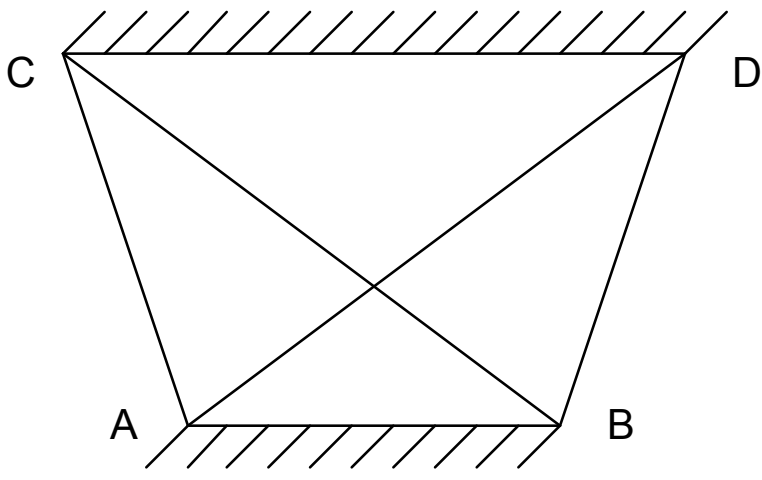

L1

Figure 3.6.4 Representation of Hottel's "String Rule" 
The shape factor, is determined using the following equation [4]:

$$
F_{12}=\frac{1}{2 L_{1}}[A D+B C-A C-B D]
$$

where the diagonal distances $\mathrm{AD}$ and $\mathrm{BC}$ along with the side distances $\mathrm{AC}$ and $\mathrm{BD}$ are to be evaluated as the lengths of strings stretched tightly across the vertices, hence the name the "string rule".

The main issue concerning this method is that these lengths change depending on where the strand lies on the nozzle plate and the distance from the surface of the strand to the coils in a direction normal to the surface for the entire 360-degree circumference of the strand. To eliminate this concern an average distance from the surface of the strand to the coils was found simply by taking the largest possible distance $(38.75 \mathrm{~cm})$ and the shortest possible distance $(5$ $\mathrm{cm})$. Therefore an approximate distance normal from the strand surface to the coils becomes 22 $\mathrm{cm}$. From here the vertical position and size of the strand section can be used to determine the distances between vertices.

The second condition acting on the control volume is the heat transfer through convection. The heat transfer coefficient, $h$, is determined by first analyzing the shape of the volume. In the case of fiberglass, sections of the glass strand will take the shape of a cylinder with a length of $\Delta \mathrm{X}$ and a diameter equal to the average of the two diameters.

Convective heat transfer is heavily dependant on a dimensionless number called, the Reynolds Number. This number is used to determine the type of flow, laminar or turbulent, over the surface of the control volume. Heat is transferred more rapidly in association with turbulent flow because of the rapid churning of the fluid over the surface. Higher Reynolds numbers usually indicate more turbulent flow. From before, the equation for the Reynolds number is [4]:

$$
\mathrm{Re}=\mathrm{VD} / \mathrm{v}
$$


Inside the Radiative hood the velocity of the cross breeze becomes reduced. The model takes this into consideration by linearizing the velocity value from zero to one hundred percent of the velocity used outside the hood based on the distance from the furnace.

Another mode of heat transfer examined will be conduction. The rate of heat transferred is based on physical properties of glass, cross-sectional area, and the temperature difference of the control volume. The rate of heat transfer through conduction can be described by the equation [4]:

$$
q=-k A \frac{\partial T}{\partial x}
$$

However, when dealing with fiberglass the cross-sectional area becomes very small (the diameter is measured in microns), therefore the conduction in the system can be neglected, and the main source of energy entering the control volume comes from the mass transfer entering the system.

In order to illustrate the magnitude of the modes of heat transfer on the problem a theoretical situation was analyzed. In this particular situation the strand diameter has decreased by one half of its total attenuation to a diameter of 0.002 meters. This theoretical control volume has been strained to a length of 0.0011 meters. The temperature of glass entering the system can be estimated at $1102 \mathrm{~K}$ and the Temperature exiting the system can be estimated at $1098 \mathrm{~K}$ with an average of $1100 \mathrm{~K}$. The ambient temperature of the air is approximately $290 \mathrm{~K}$ and the temperature of the coils in the radiative hood is approximately $310 \mathrm{~K}$. For this situation the heat transfer rate for convection becomes approximately $664 \mathrm{~W}$; radiation is approximately $275 \mathrm{~W}$; and conduction is approximately $0.01 \mathrm{~W}$. As can be seen the heat transfer rate of conduction is dwarfed by the rates for convection and radiation. This is because of the very small dimensional 
properties of the control volumes. Because of the small diameters, most of the control volume is surface area.

This concept also holds true for the Biot Number. The high heat transfer coefficients caused by convection and the small diameters associated with conduction make the Biot Number small enough to assume an evenly distributed core temperature across the cross-section of the strand. In the situation examined above the Biot number is approximately 0.083 , which is less than the 0.1 criteria for constant core temperature.

It is now possible to determine the equation that will govern the temperature profile in the model. Rewriting equation (3.6.3) using formulas for heat transfer discussed in the literature review and model specific variations gives:

$$
\dot{m} C p T_{\text {in }}=\dot{m} C p T_{\text {out }}+h A\left(T_{\text {avg }}-T_{\infty}\right)+\sigma \varepsilon A F_{12}\left(T_{\text {avg }}^{4}-T_{\infty}^{4}\right)
$$

In order to get the equation in terms of $T_{i n}$, the temperature of glass entering the control volume, and $\mathrm{T}_{\text {out }}$, the temperature of glass exiting the control volume, only, $\mathrm{T}_{\mathrm{avg}}$ can be replaced with:

$$
T_{\text {avg }}=\frac{\left(T_{\text {in }}+T_{\text {out }}\right)}{2}
$$

The temperature, $\mathrm{T}_{\infty}$, used in the radiation portion of the equation will be set equal to a recorded temperature of the coils and referred to as $\mathrm{T}_{\mathrm{c}}$. Rewriting the equation in terms of $\mathrm{T}_{\text {in }}$ and $\mathrm{T}_{\text {out }}$, the following formula is obtained:

$$
\dot{m} C p T_{\text {in }}=\dot{m} C p T_{\text {out }}+\left[h A\left(\frac{T_{\text {in }}+T_{\text {out }}}{2}-T_{h}\right)\right]+\sigma \varepsilon A F_{12}\left(\frac{\left(T_{\text {in }}+T_{\text {out }}\right)^{4}}{16}-T_{c}^{4}\right)
$$

The final model uses a numerical analysis method to determine the temperature of the glass along the strand. After the new length and diameter is determined, the new section will be approximated using a cylinder with a diameter equal to the average of the two ends. Because of 
the fourth order terms that result from the radiation equation, it is extremely difficult to solve the equation. Therefore an iterative numerical method is needed to solve this system of equations.

The method used was discussed in Dr. Çelik's book [7] and is known as the Jacobi Iteration Method for Non-linear equations. The simple case of two equations and two variables will be looked at to better explain the Jacobi Iteration Method. In this case the two equations need to be rewritten in the following form:

$$
x=f(x, y)
$$

For more variables and equations continue with the same general idea with one equation for each individual variable. The heat transfer equation for the model only involves one equation and one unknown $\left(\mathrm{T}_{\text {out }}\right)$. To use this in an iterative procedure the above equation must be written in the following form:

$$
x_{i+1}=f\left(x_{i}, y_{i}\right)
$$

The method works by giving the variables an initial guess value (in this case $\mathrm{x}_{0}$ ), entering these guesses into the equation $(\mathrm{f}(\mathrm{x}, \mathrm{y}))$, and determining a new value to replace the initial guesses. The process continues until the new value and the guess value differ by as small of a percentage as designated by the user.

Initial guesses for this iteration process will be equal to the temperature of the glass entering the control volume, because a fourth order system has 4 roots and the needed root has to be a real, positive value close to the initial temperature.

In order to get the heat transfer equation in a form usable by the iterative method it must be rewritten in a form where the exiting temperature is isolated on one side of the equation. The new heat transfer equation becomes: 


$$
\begin{aligned}
& T_{\text {out }}= \frac{\dot{m} C p T_{\text {in }}-\frac{\sigma \varepsilon A F_{12}}{16}\left(T_{\text {in }}^{4}+6 T_{\text {in }}^{2} T_{\text {out }}^{2}+4 T_{\text {in }} T_{\text {out }}^{3}+T_{\text {out }}^{4}\right)}{+\sigma \varepsilon A F_{12} T_{c}^{4}+h A T_{\infty}-\frac{h A}{2} T_{\text {in }}} \\
& \dot{m} C p+\frac{\sigma \varepsilon A F_{12}}{4} T_{\text {in }}^{3}+\frac{h A}{2}
\end{aligned}
$$


This page intended to be blank. 


\section{Results}

\subsection{Model Outputs}

Once the code is completed, outputs must be validated. To do this data will be compared with visual and physical data approximations collected from the company and its employees. A standard model will be developed using data collected on an average operating day.

For the standardized model an ambient air temperature of $300 \mathrm{~K}$ will be used. The furnace temperature will be $1384 \mathrm{~K}$, and the coil temperature will be $320 \mathrm{~K}$. The coil temperature comes from a digital reading on the surface of the coils. Running the drums at 149 RPM created an average strand diameter of 28.5 microns. This number will be used to determine flow rates of the nozzles along with the final diameter of the standardized model.

The following graph, Figure 4.1.1, shows the diameter of the strand as a function of the distance from the furnace. As can be seen, elements characteristic of all attenuation models are present. As the glass exits the nozzle it immediately begins an area of rapid diameter reduction, followed by an exponential change in reduction rate forming what is known as a meniscus.

Examination with the naked eye gives an estimation of the meniscus length be around 2 inches, or $4.68 \mathrm{~cm}$. Figure 4.1 .1 shows the end of the meniscus to be around 7 centimeters, which could prove problematic. The approximation was made without the use of magnification, however, and the strand diameter at 2 centimeters is less than 0.5 millimeters. Discerning diameter changes at this size would be almost impossible without magnification, therefore the graph can still be considered reasonable. 


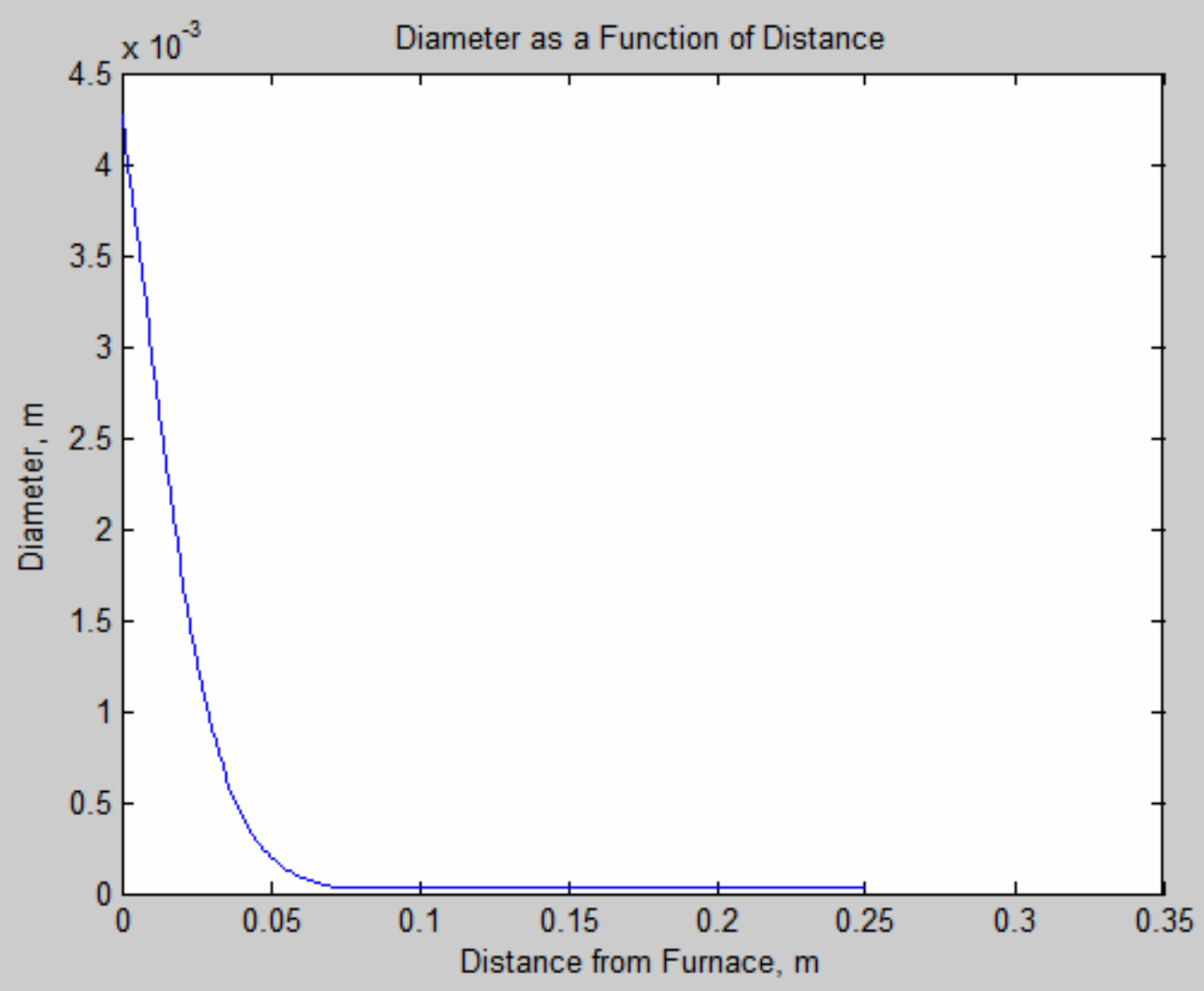

Figure 4.1.1 Strand Diameter as a Function of Distance from the Furnace

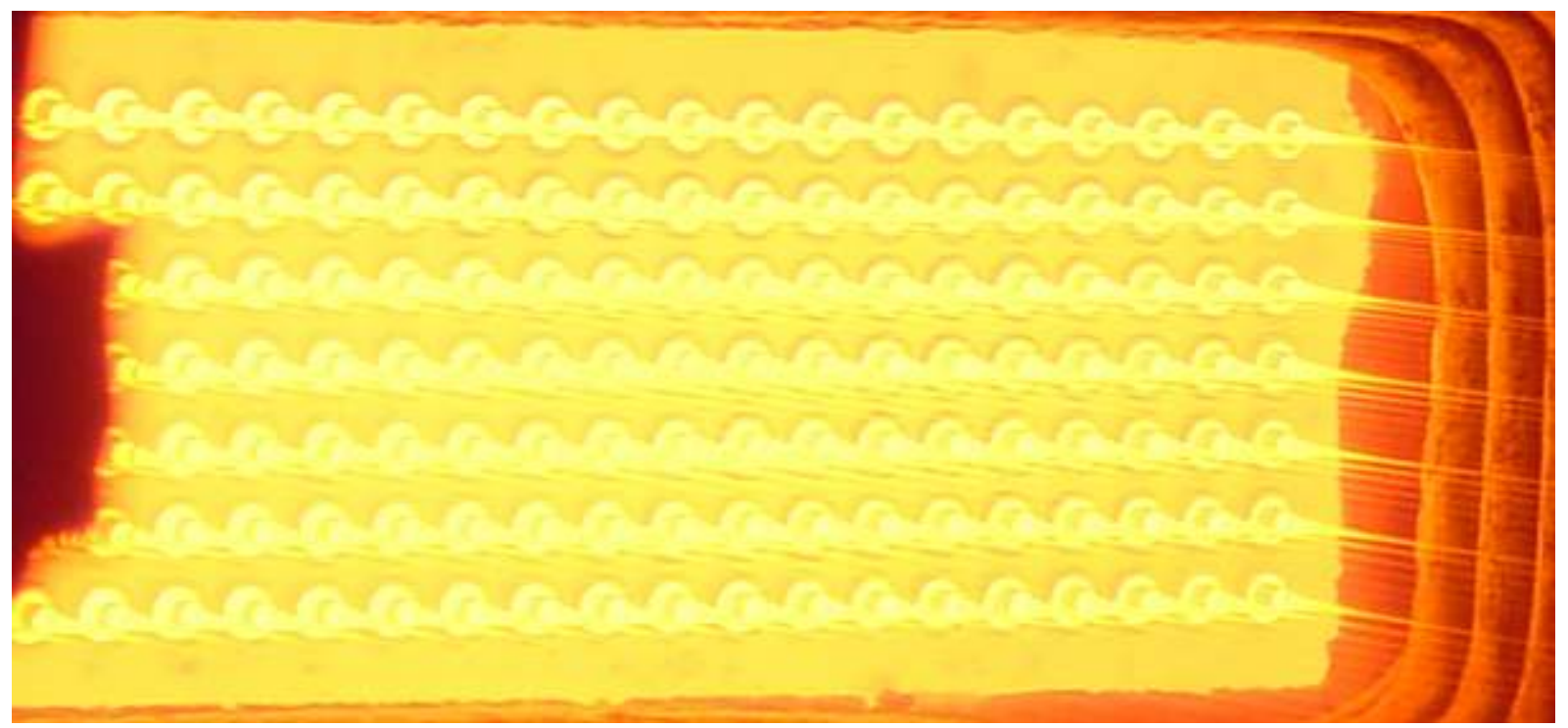

Figure 4.1.2 View of nozzle plate, coils, and glass meniscus

Another number checked for its plausibility is the force applied to the strands to pull them onto the drum. The guess and check method is used to determine the force needed to pull 
the strand to the required diameter based on drum speed. Workers claimed that less than one pound of force was required to pull down the 294 strands. At 149 RPM the force on an individual strand was determined by the model to be $0.0031932 \mathrm{~N}$ or $0.939 \mathrm{~N}$ for all 294 strands, which is equivalent to slightly less than 0.25 pounds of force. This number concurs with the workers claiming a very small amount of force needed to draw the strands.

The model also has the ability to display core strand temperature as a function of distance from the furnace. This allows analysis of heat transfer to be performed. Figure 4.1.3 shows this temperature distribution for the standardized model.

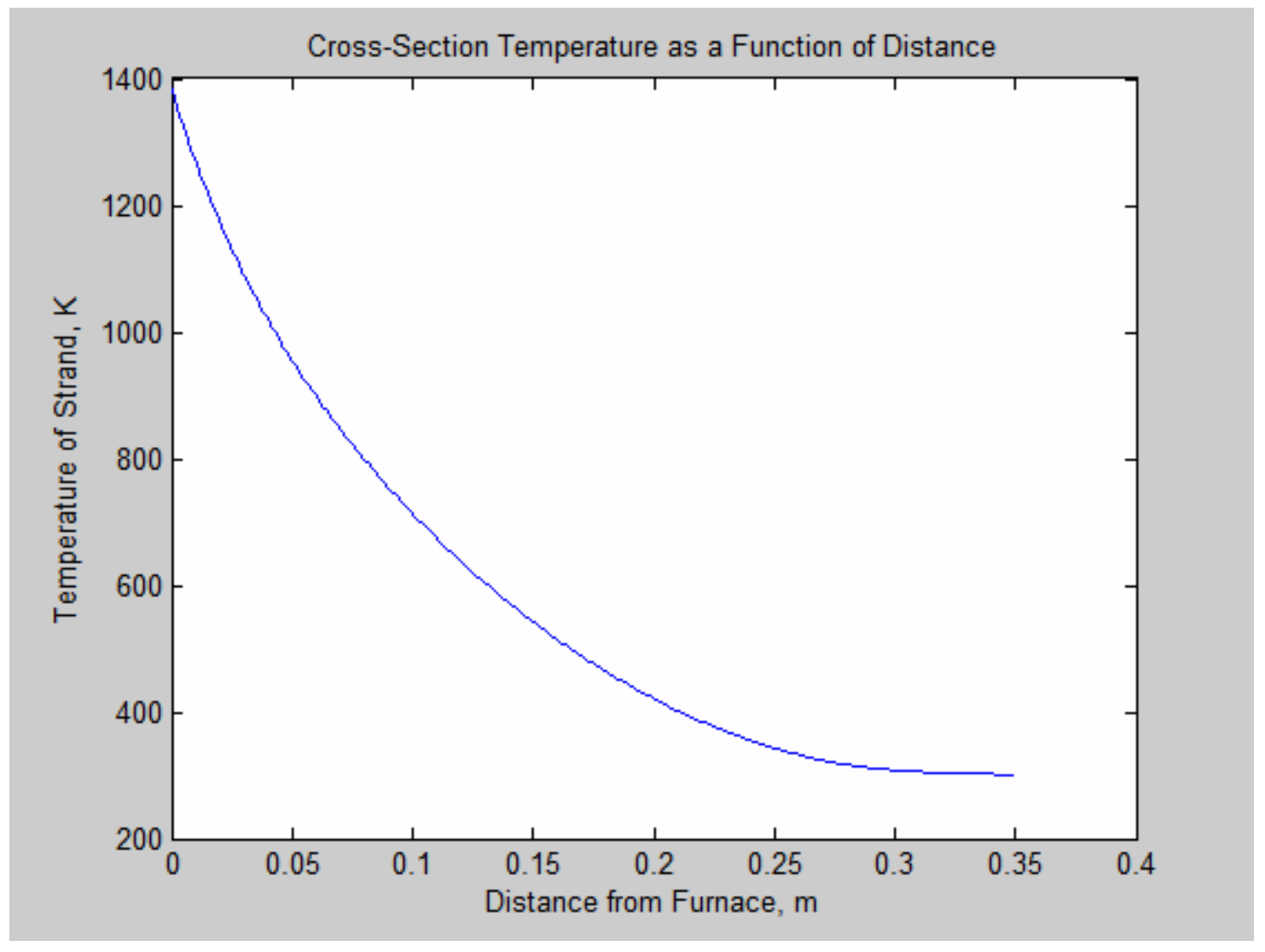

Figure 4.1.3 Cross-Section Temperature as a Function of Distance (assuming cross-section temperatures constant based on Biot Numbers)

The shape of the curve is what would be expected: a larger decrease in temperature at higher cross-sectional temperature with a more gradual decrease as temperature approaches 
ambient temperature. The small diameter of the strands allows for most of the strand to be surface area. This causes rapid cooling. The MATLAB program has an internal check for the Biot Number, which coincidentally never exceeded the 0.1 used to assume that the internal temperature of the glass is constant throughout. The temperature of the glass reaches ambient temperature quickly. This can be verified by the workers' ability to handle the glass without protective gloves shortly below the radiative hood.

Now some of the controllable variables will be looked at to determine how these variables affect the outcome of the strand. The first variable analyzed will be the speed of the drum. The model is calibrated to $149 \mathrm{rpm}$, the current plant condition. Two other drum speeds, one higher and one lower, were then used to generate a plot of the strand diameter as a function of distance from the furnace.

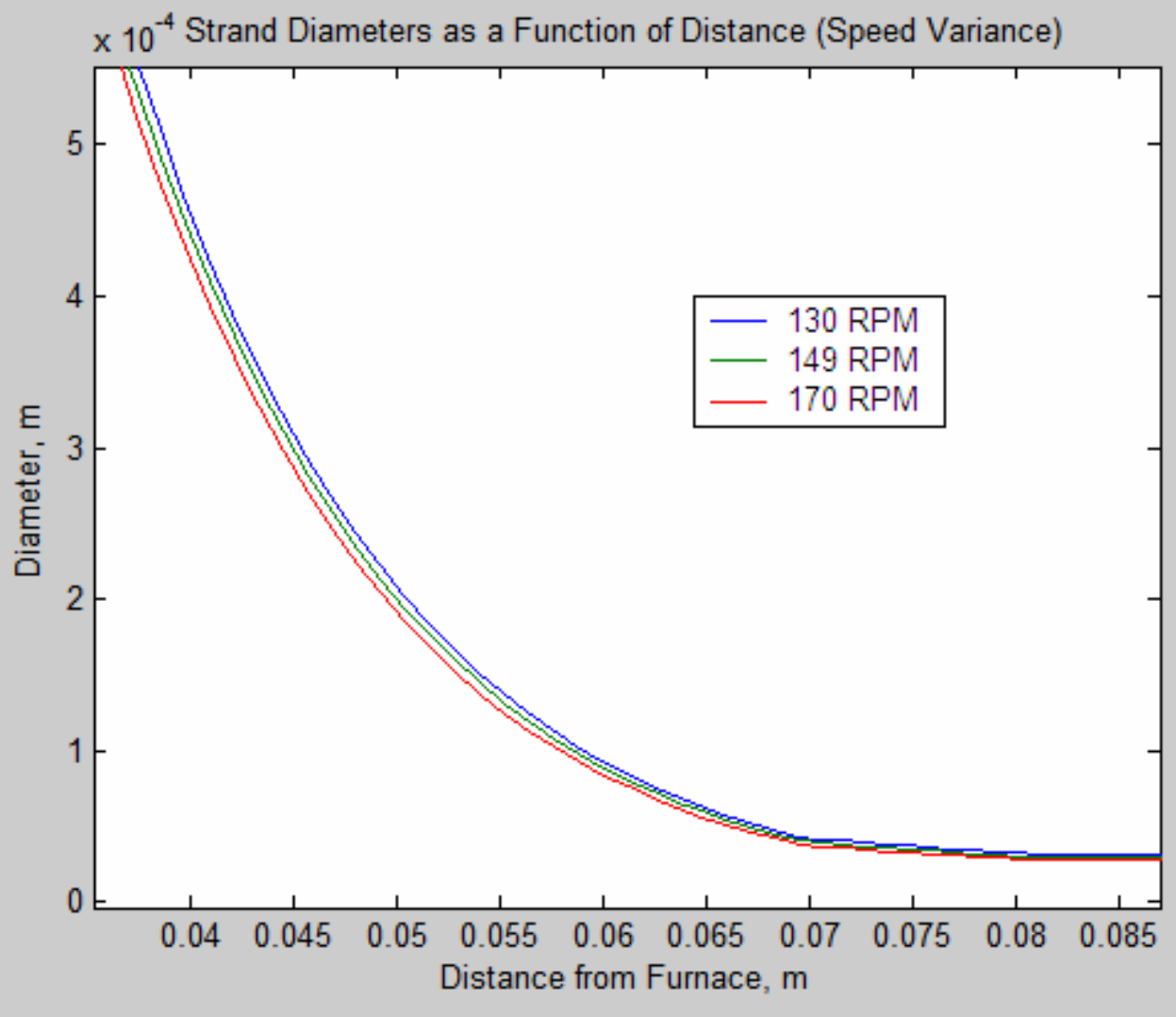

Figure 4.1.4 Strand Diameter with Speed Variance 
As can be seen in the graph, speed variance has an effect on the diameter as a function of distance from the furnace. Higher drum speeds cause a quicker decline in diameter. As the strands become closer to their final diameters, the difference in strand diameter as a function of the distance from the furnace becomes smaller. These diameters, however, never regain equality. Higher drum speeds yield smaller diameter strands. The affect of drum speed on the final diameters (i.e., a more in depth view of the data found in Figure 4.1.3 between the range of 0.08 and $0.085 \mathrm{~m}$ ) can be found in Appendix 1 .

The concentration of stress varies along the axis with the changing diameter. Figure 4.1.5 was made using the standard model parameters (149 RPM, 300K ambient temperature, etc.) and illustrates the change in stress along the axis as a function of distance from the furnace. This graph shows the maximum tensile stress acting on the strand to be at the base of the meniscus where the diameter is the smallest. Because this stress is the largest, the stress at the base of the meniscus will become the basis for all stress analysis. Varshneya (p. 409) [3] lists the strength of freshly drawn glass fibers to be about 0.7-2.1 GPa. As can be seen in the graph, the stress on the glass is well below this limit when the glass is under normal conditions. 
Stress as a Function of Distance from the Nozzle

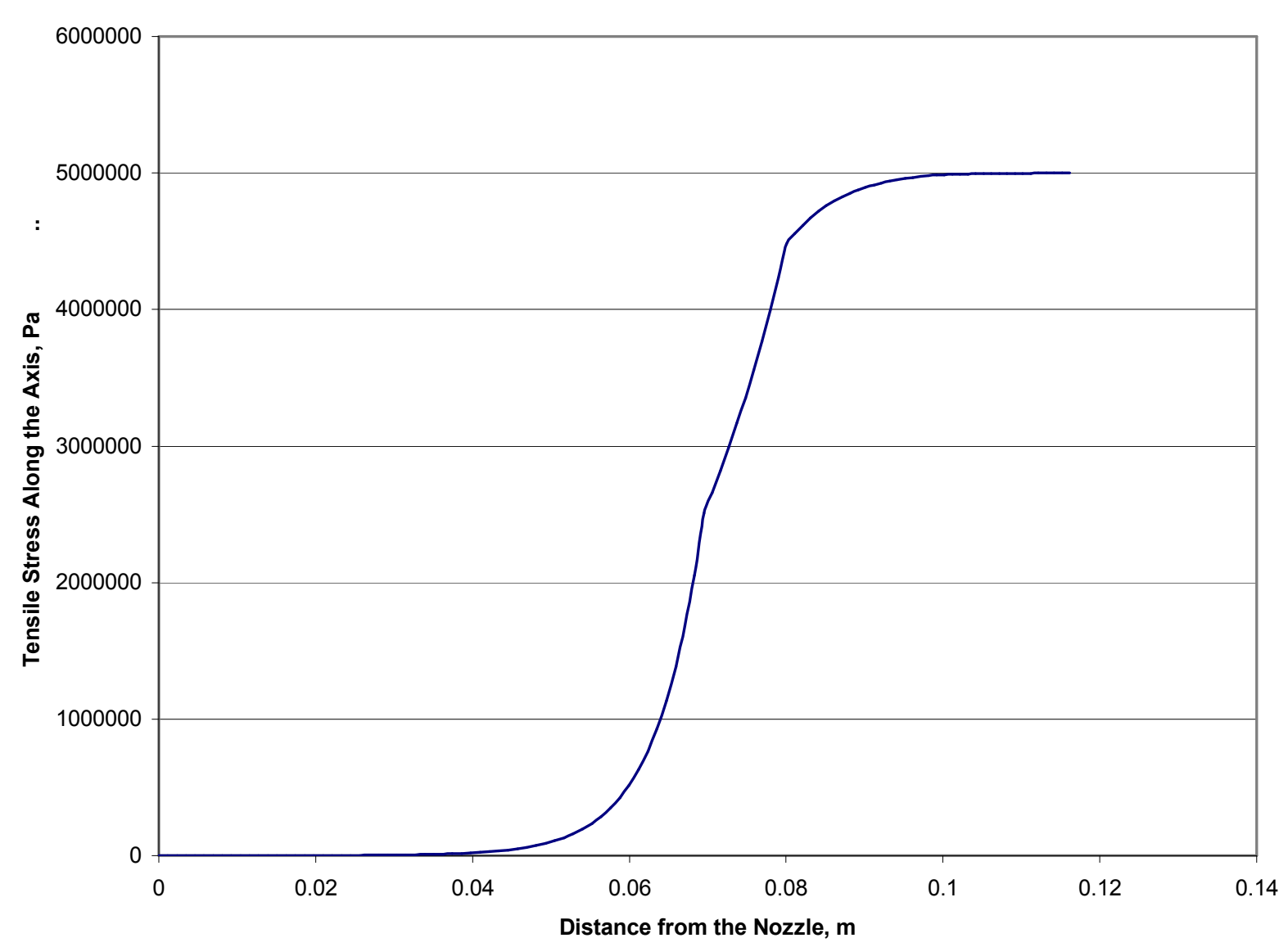

Figure 4.1.5 Stress as a Function of Distance from the Nozzle

Perhaps a more important question than how stress is distributed throughout the meniscus of the strand is how changes in drum speed affect the stress on the strand. Figure 4.1.5 shows the maximum stress at the base of the meniscus of the strands as a function of drum speed. 
Stress as a Function of RPM

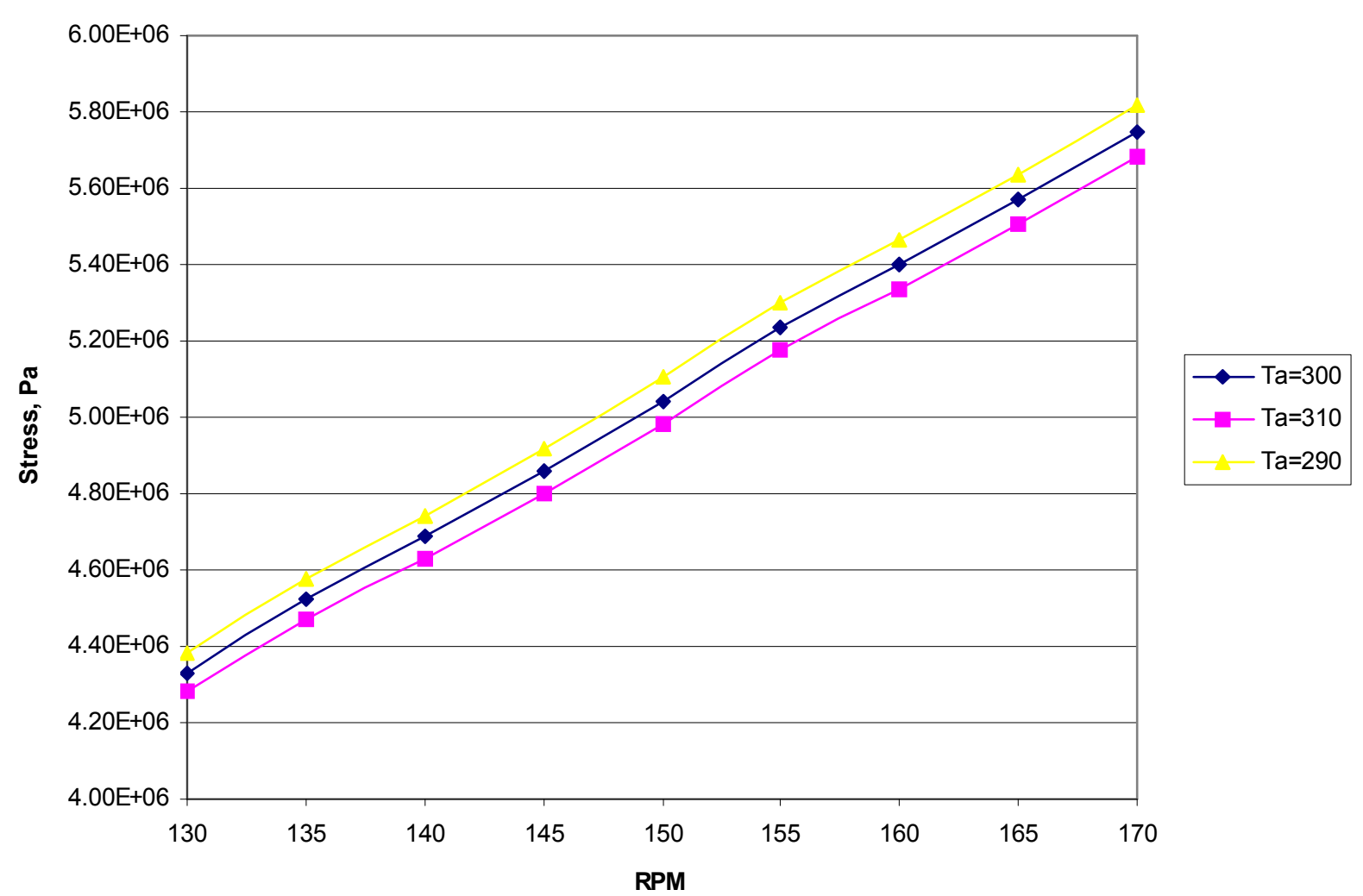

Figure 4.1.6 Stress as a Function of RPM at Three Varying Ambient Temperatures

The graph shows a linear increase in stress with the increase of drum speed. As was expected, based on Loewenstein's claim that faster attenuation rates yield a larger chance of breakage, higher drum speeds yield higher stresses in the strands. The figure also shows a comparative view of how ambient temperature affects stress. 
Stress as a Function of Ambient Temperature

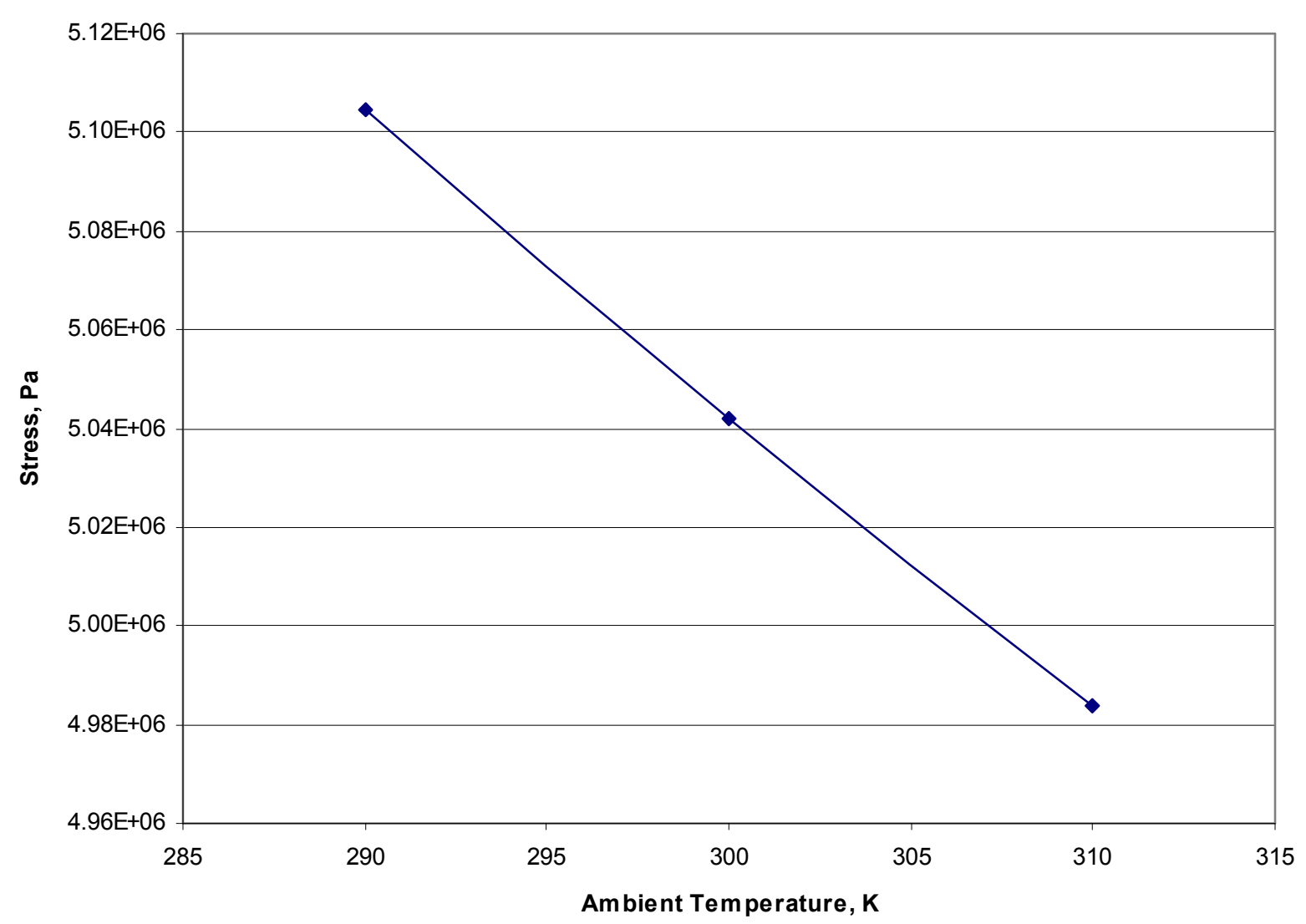

Figure 4.1.7 Stress as a Function of Ambient Temperature

Figure 4.1.7 shows more closely how ambient temperatures affect the stress on the strand. The data range covers a range of average ambient temperatures: $290 \mathrm{~K}$ to $310 \mathrm{~K}\left(17^{\circ} \mathrm{C}\right.$ to $\left.37^{\circ} \mathrm{C}\right)$. Over this range the change in stress appears to be linear. It has already been shown that higher temperatures yield lower stress. Because heat transfer rates depend on the temperature difference between the two bodies exchanging heat, higher ambient temperatures would lead to lower heat transfer rates. Like before, lower heat transfer rates keeps the glass in a state more susceptible to strain for a longer period of time requiring less tensile force, and also less stress, to strain glass to the final diameter. 
Because strand breakage seems to occur more during hot summer months, it could be assumed higher ambient temperatures result in higher stresses. On the contrary, the graph shows that higher ambient temperatures result in lower stresses.

An overlooked root cause of breakage is the small impulse deviations in force. These deviations, from here on known as "noise" forces, occur as a result of many different sources. For example drag force from cross breeze, airborne binder striking the strand, or an acceleration or deceleration of the traversing furnace are possible causes of noise force.

\begin{tabular}{|l|l|l|l|l|l|} 
Ambient & Ideal Stress, & $\begin{array}{l}\text { Stress with } 0.00001 \mathrm{~N} \\
\text { of "Noise" Force } \\
\text { added }\end{array}$ & Stress Increase, & of "Noise" Force & Stress \\
Temp., Ka & $\mathrm{Pa}$ & added & Increase, Pa \\
\hline $\mathrm{Ta}=290$ & $5.0668 \mathrm{E}+06$ & $5.3478 \mathrm{E}+06$ & $2.8102 \mathrm{E}+05$ & $8.6559 \mathrm{E}+06$ & $3.5891 \mathrm{E}+06$ \\
$\mathrm{Ta}=300$ & $5.0048 \mathrm{E}+06$ & $5.2890 \mathrm{E}+06$ & $2.8421 \mathrm{E}+05$ & $8.6483 \mathrm{E}+06$ & $3.6436 \mathrm{E}+06$ \\
Ta=310 & $4.9467 \mathrm{E}+06$ & $5.2335 \mathrm{E}+06$ & $2.8680 \mathrm{E}+05$ & $8.6449 \mathrm{E}+06$ & $3.6981 \mathrm{E}+06$
\end{tabular}

Table 4.1.1 The Result of Noise Forces

Table 4.1.1 shows the stress on the strands under ideal conditions for a drum speed of 149 RPM at three different ambient temperatures. An arbitrary noise force equaling $0.00001 \mathrm{~N}$ was then added to the tension of the strand. After the tensile force was added in the code the strand diameters became smaller. A new tensile stress was determined with the new force and a comparison of the ideal stress and the increased stress was made. The table shows a higher increase in added stress caused by the noise force at higher ambient temperatures. Eventually larger noise forces will cause the stress on the strands at higher ambient temperatures to exceed the stress on the strands with lower ambient temperatures leading to a higher probability in 
breakage. The stresses on each strand after the $0.0001 \mathrm{~N}$ of noise forces are almost equal. The strands with the highest ambient temperatures continue to see the greatest increases in stress.

The magnitude of these noise forces can be justified by determining the weight added to a strand after airborne binder struck it. Assume an airborne bead of binder has a diameter of one millimeter and its density is the same as water (due to water being its main ingredient). By finding the kinetic energy of an airborne binder and assuming the binder hits the fiber squarely and splatters giving up half its energy to the fiber while causing it to deflect one centimeter, the force can be found with the following equation:

$$
1 / 2 m V^{2}=F(0.01 m)
$$

Using geometry with these assumptions, a tensile force value of approximately $6.25 \times 10^{-4} \mathrm{~N}$ was determined. This value is more than six times the highest value in table 4.1.1. Noise forces of this magnitude would show an even larger difference in the different increased stresses. For the standard condition model (149 RPM, ambient temperature of $300 \mathrm{~K}$ ) adding this much force shrinks the diameter of the strand to approximately 4 microns and generates stress at approximately $2.96 \times 10^{8} \mathrm{~Pa}$. This is still below the strength listed in Varshneya[3]. However, raising the ambient temperature to $310 \mathrm{~K}$ yields strands approximately 2.5 microns in diameter and a stress approximately $0.79 \mathrm{GPa}$. This stress is above Varshneya's [3] limit. This becomes definite proof of the importance of controlling the ambient temperature.

Figure 4.1.8 helps to better understand the effect of temperature on the strand and its importance. The graph shows a realistic plot of the strand diameter at the base of the meniscus as a function of time. The lines represent two different strands acted on by identical forces at the same time. One strand exists in high ambient temperature conditions, while the other is exposed to lower ambient temperatures. 
Theoretical Meniscus Base Diameter as a Function of Time to Illustrate Effect of "Noise" Forces and Temperature

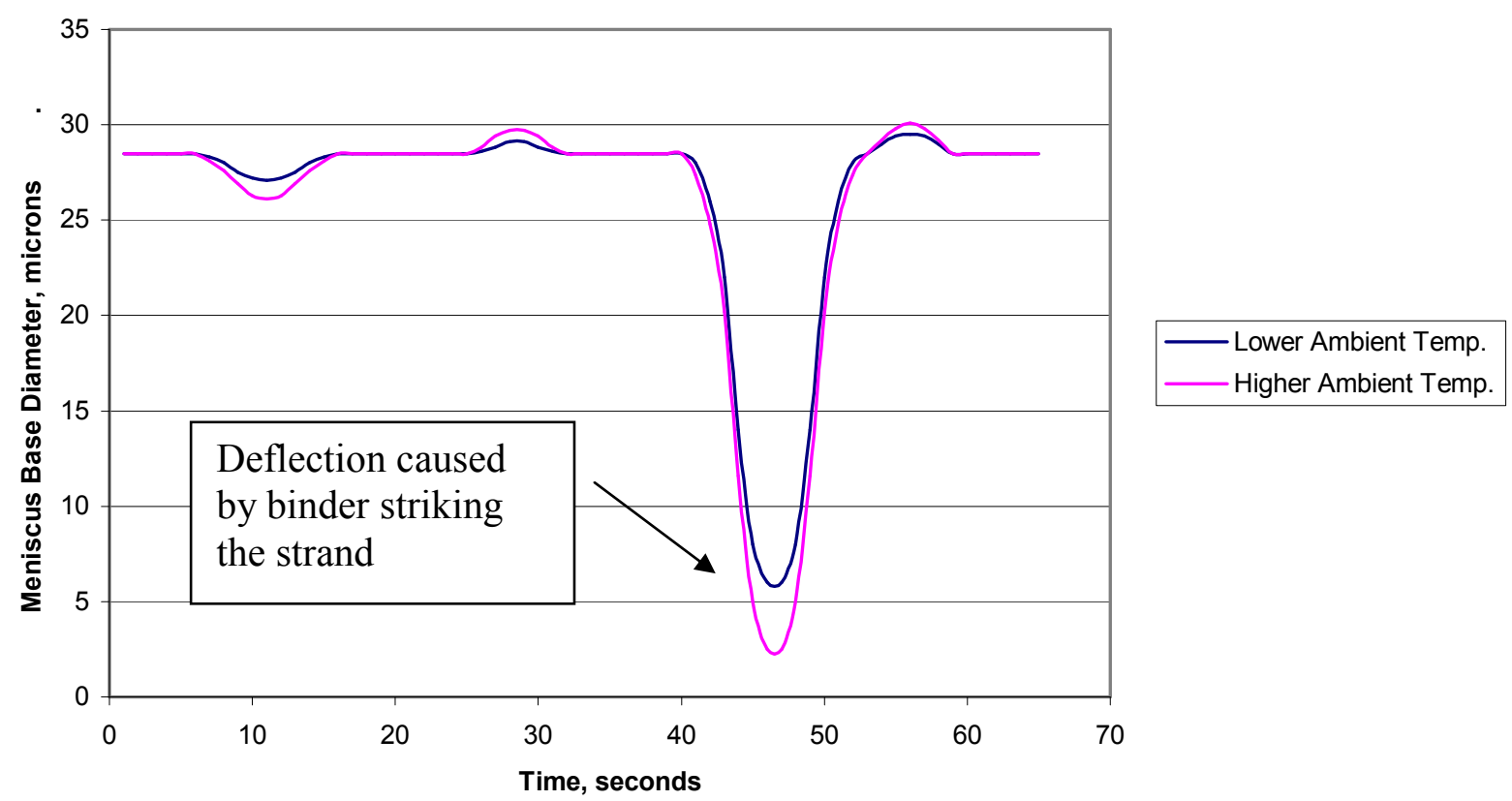

Figure 4.1.8 Theoretical Meniscus Base Diameter as a Function of Time to Illustrate Effect of "Noise" Forces and Temperature

As can be seen in the graph, the average diameter in both cases is equal. However, instances where the strand was affected by outside forces, particularly binder striking the strand, the strand exposed to higher ambient temperatures suffered greater deformation due to stress than the strand exposed to lower ambient temperatures and consequently higher heat transfer rates. Because the tensile force is constant throughout the length of the strand, a smaller diameter exhibits a much higher stress. In the case of the binder striking the stand, the stand with lower ambient temperatures will have an increased chance of not exceeding the strength of the glass.

Another temperature controllable variable, other than ambient temperature, is the temperature of the glass directly above the plate inside the furnace. Adjusting the flow rate of burning gas inside the furnace controls this temperature. Figure 4.1.9 shows where the 
maximum stress on the strand is affected by the furnace temperature. This model uses the standard 149 RPM drum speed in its calculations.

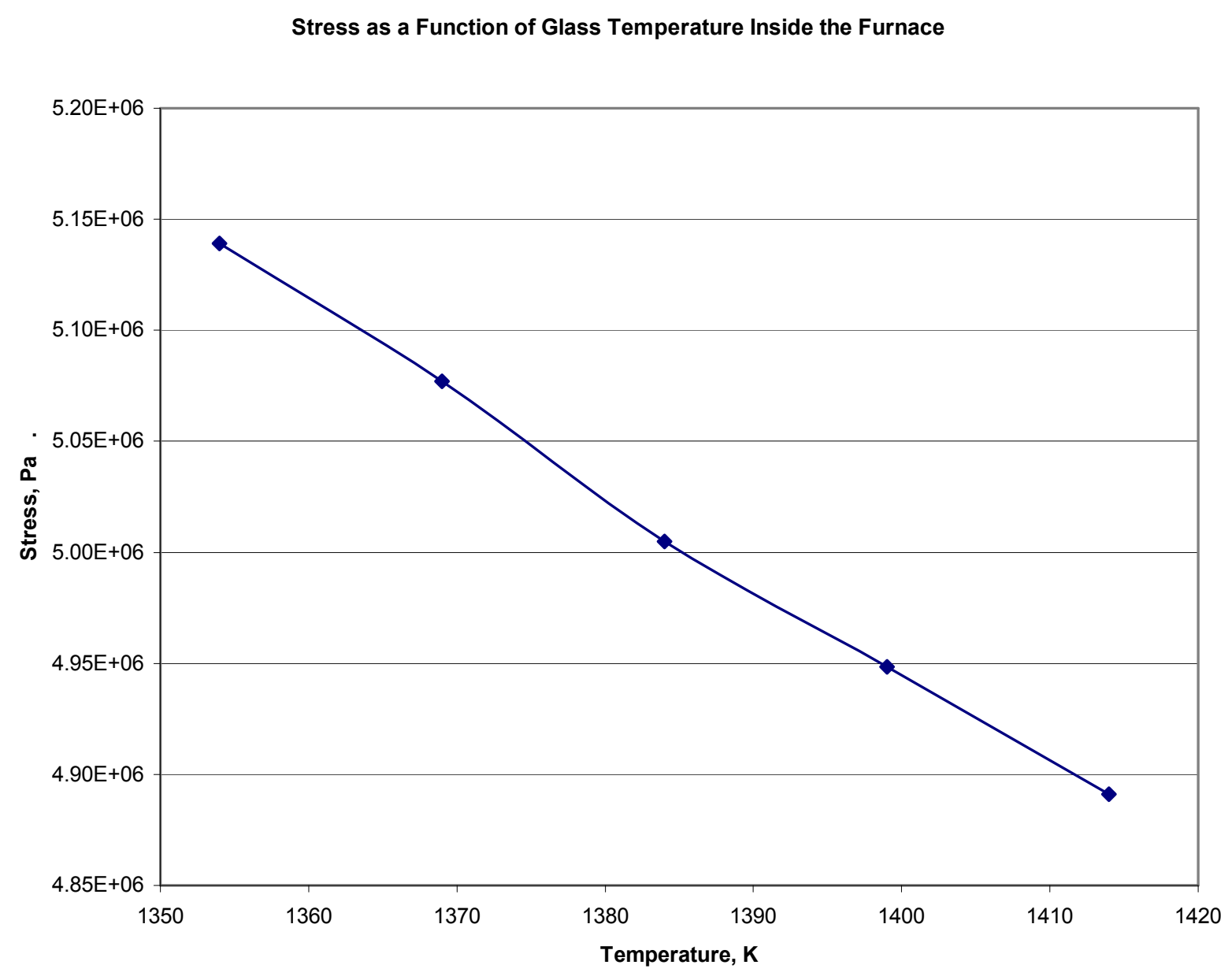

Figure 4.1.9 Stress as a Function of Glass Temperature inside the Furnace

This graph shows that it is possible to adjust the stress acting on the strand by adjusting the furnace temperature. Increasing the furnace temperature decreases the amount of stress on the strand. This occurs because glass requires less stress to obtain a specific strain rate in the liquid phase than the viscoelastic or solid phases. Therefore, keeping the glass in this phase for an extended period of time would result in the need for lower tensile force and its corresponding stress and strain rates. Unfortunately there is a negative result to increasing the temperature of 
the glass inside the furnace. Based on the theory of "noise" forces, an increased temperature at the beginning of the strand would result in the glass remaining in the liquid state for an extended period of time and thus increasing the effect of "noise" forces on the strands.

It is impractical to concentrate on adjusting the furnace temperature as a primary means of controlling the stress in the strand for many reasons. One reason control of this temperature is impractical is the time it takes for the glass to distribute the heat reaching a steady state. Minor adjustments in the furnace temperature take hours to redistribute heat.

It can be seen in Figure 4.1.9 that a temperature change of $60 \mathrm{~K}$ only creates a change of five percent in the stress. Substantially decreasing the temperature of glass inside the furnace leads to different flow rates inside the nozzle, which has detrimental effects on the final product. From the equation for nozzle flow, the only temperature dependent variable is the viscosity of molten glass. However, it was shown in figure 2.2.1 that the viscosity above a certain temperature, approximately 1000 degrees Kelvin, is relatively constant. This means that temperatures above this will result in equal flow rates. Trying to finely adjust the viscosity around the point were the viscosity begins to change is impractical due to the difficulty of positioning a measuring device directly over the nozzle. Also maintaining an equal temperature distribution over the entire nozzle plate would be extremely difficult.

Although adjusting the temperature inside the furnace has some affect on the stress in the strands it is small in comparison to the affect ambient temperature has on strands. It would make more sense for the company to concentrate on developing ways to more carefully control the ambient temperature and radiative heat transfer via the coils than it would to focus on controlling the temperature inside the furnace. 


\subsection{Conclusions}

There are several factors that affect the attenuation of fiberglass. How these parameters affected the process was examined in this thesis. The model's outputs were justified by comparing theorized data with observations made at the factory. Note that precise measurements were not taken at the subject plant to compare the overall accuracy of the model. The model's output was only compared to observations justifying its ability to predict trends.

The variable most contributing most to the shape of the glass is the design of the nozzle. The flow rate coming out of the nozzle is independent of ambient temperature, drum speed, etc. As the drum speed increased, the velocity of the strand at the drum increased. Because the flow rate maintains a constant value, due to conservation of mass principles, the cross-sectional area of the glass must decrease to accommodate the higher velocities.

As was shown in section 4.1, temperature did not affect the final shape of the glass only the attenuation. When higher temperatures are involved anywhere in the system (e.g., higher ambient temperatures, higher furnace temperature, and higher coil temperatures) the glass was maintained in a state more susceptible to strain for a longer period of time. This means that any random external forces acting on the strand, such as airborne binder striking the strand, caused the strand to strain more than it would in situations where it was exposed to lower temperatures. Basic stress analysis says if two objects of identical material are exposed to equal forces the object with the smaller cross-sectional area will have a greater level of stress than its larger counterpart. This concept reflects the importance of temperature control in the system; not as a means of determining the final product, but increasing the strands likelihood of withstanding external stress. 


\subsection{Suggestions for Higher Efficiency}

Glass at lower temperatures, particularly in the solid phase, is more resilient to strain than glass at the viscoelastic stage and especially the liquid stage. So it would make sense that the quicker the glass reaches these phases the more resistant it will be to extra unpredictable stresses. Methods could be determined to aid the plant in maintaining a constant, optimized ambient temperature year round.

One of the theorized causes of breakage is sling. Sling occurs when a special binder sprayed on the drum is literally slung through the air occasionally striking a glass strand. It has been proven that small beads of sling can have a significant effect on the stress on an individual strand, especially when heat transfer rates are their smallest. Because of this it can be suggested that reducing the probability of sling striking a strand would greatly reduce the probability of breakage. A possible, easy solution would be an extended hood capable of blocking more sling. There is also the possibility of controlling the amount of binder sprayed.

Another good idea would be a further study of the properties of the binder. This would enable the plant to establish a means of controlling certain parameters like viscosity, surface tension, and general tackiness, which are all dependent on temperature. If installing an inexpensive, strategically placed fan to cool the binder would drastically decrease the amount of sling, the benefits could far outweigh the cost.

Another possible solution is redesigning the nozzle. Figure 4.1.4 shows that slower drum speeds exhibit a lower force on the strands. The subject company could look into optimizing the balance between drum speed and the downtime caused by breakage. Lowering the drum speed, however, has a substantial effect on the final product. Loewenstein states that increasing drum 
speed does not increase or decrease weight-based production rate, only the final strand diameter. Therefore, in order to lower drum speeds and maintain the consistency of the final product, adjustments to the nozzle must be made, particularly the diameter, or height of the glass inside the furnace. 


\section{BIBLIOGRAPHY}

1. Tooley, Fay V., The Handbook of Glass Manufacture, V.1, 3 ed, Ashlee Publishing Co. Inc., New York, NY, 1984 ISBN 0-911993-20-7

2. Tooley, Fay V., The Handbook of Glass Manufacture, V.2, 3 ed, Ashlee Publishing Co. Inc., New York, NY, 1984 ISBN 0-911993-21-5

3. Varshneya, Arun K., Fundamentals of Inorganic Glasses, Academic Press, Inc., San Diego, CA, 1994 ISBN 0-12-714970-8

4. Mills, A.F., Basic Heat \& Mass Transfer, 2 ed, Prentice Hall, Inc., Upper Saddle River, NJ, 1999 ISBN 0-13-096247-3

5. Shames, Irving H. and Francis A. Cozzarelli, Elastic and Inelastic Stress Analysis, Revised Printing, Taylor and Francis, Philadelphia, PA, 1997 ISBN 1-56032-686-7

6. Çengel, Yunus A. and Michael A. Boles, Thermodynamics: An Engineering

Approach, 4 ed., McGraw-Hill, New York, NY, 2002 ISBN 0-07-238332-1

7. Celik, Ismail B., Introductory Numerical Methods for Engineering Applications, Ararat Books \& Publishing, LLC. Morgantown, WV, 2001 ISBN 0-9713403-0-7

8. Jian, Christopher Q., Simulation Delivers Significant Savings in Process Rebuild for More Environmentally Friendly Fiberglass, Journal Articles By Fluent Software Users, JA169, Available at www.fluent.com, 2003

9. “Single Glass Fiber Drawing”, Flow Modeling Example, Fluent, http://www.fluent.com/solutions/examples/x30.htm, February 9, 2005 
10. "Fiber Spinning Module Introduced in FLUENT", Reprint from FluentNEWS Spring 1997, p13, http://www.fluent.com/about/news/newsletters/02v11i1/a20.htm, February 2,2005

11. White, Frank M., Fluid Mechanics, 4 ed., The McGraw-Hill Companies, Boston, MA, 1999, ISBN 0-07-069716-7

12. Loewenstein, K.L., The Manufacturing Technology of Continuous Glass Fibres, El Sevier Scientific Publishing Company, New York, NY, 1973, ISBN 0-444-41109-7

13. Mohr, J.Gilbert and William P. Rowe, Fiberglass, Van Nostrand Reinhold Co., New York, NY, 1978, ISBN 0-442-25447-4 


\section{Appendix 1 Determining Strand Diameter}

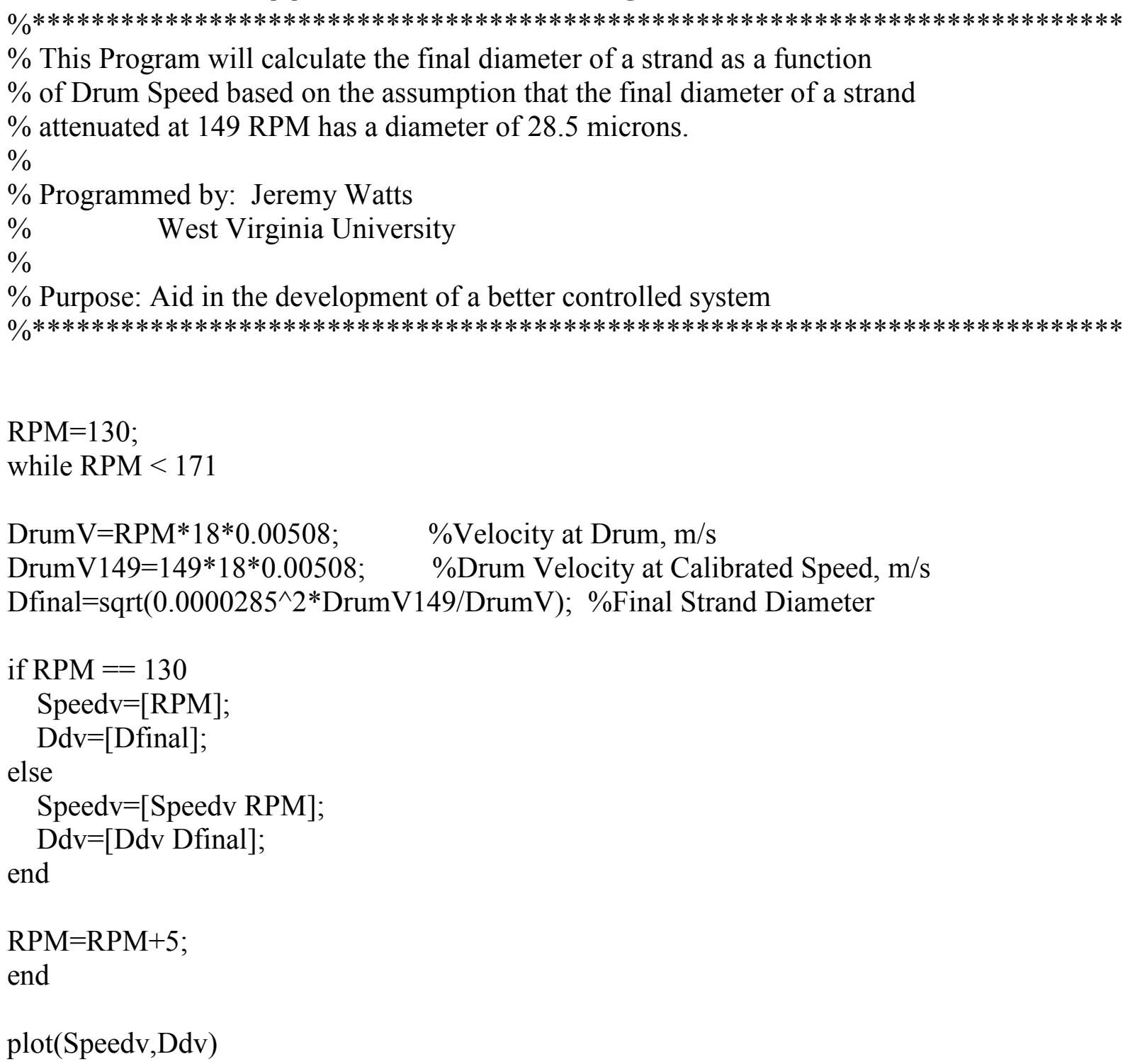




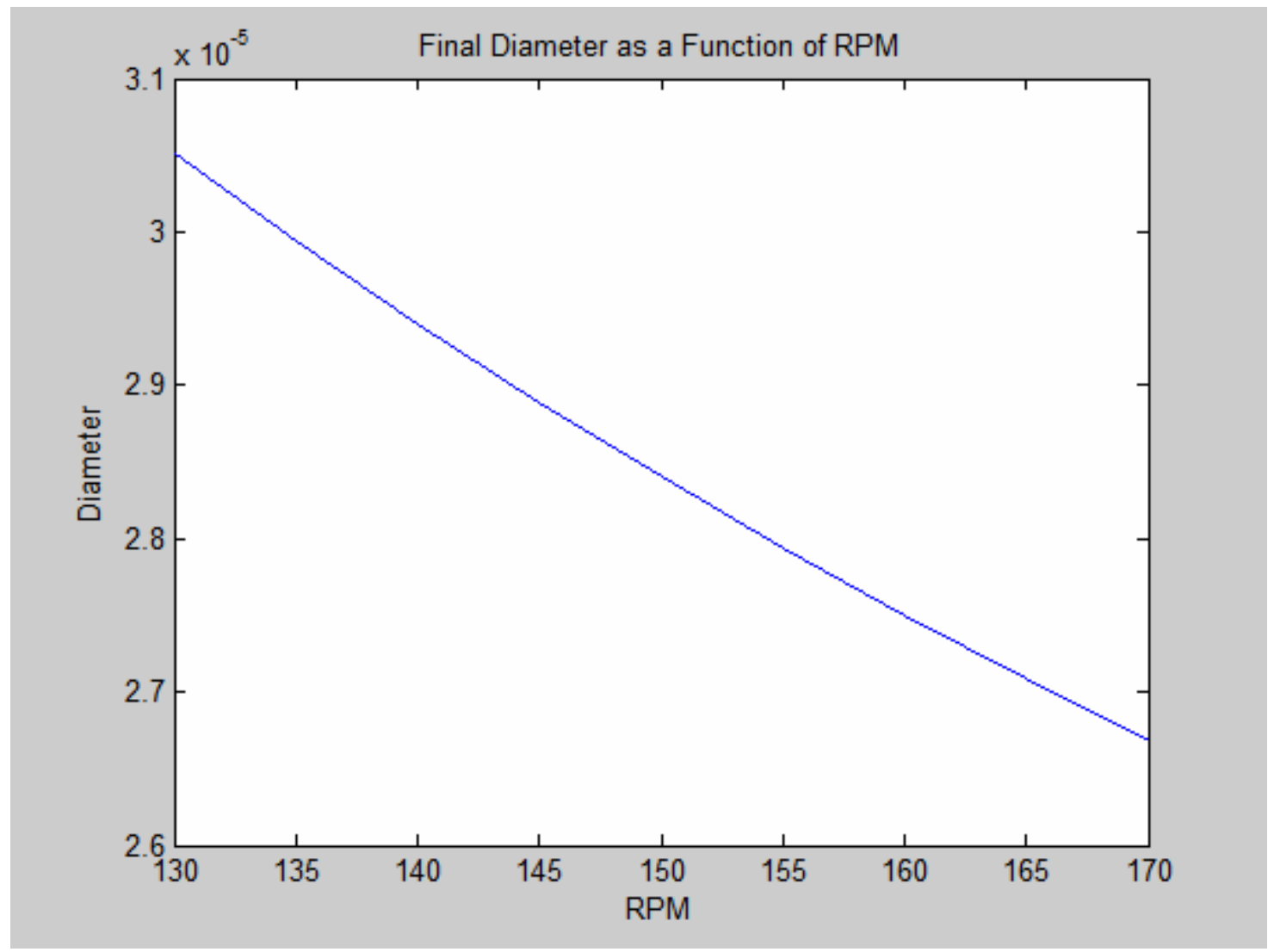




\section{Appendix 2 Modeling the Attenuation of Fiber Glass}

\% $* * * * * * * * * * * * * * * * * * * * * * * * * * * * * * * * * * * * * * * * * * * * * * * * * * * * * * * * * * * * * * * * * * * * * * * * * *$

$\%$ This Program will calculate the shape of a glass strand being drawn from

$\%$ a die and spun onto a drum.

$\%$

$\%$ Programmed by: Jeremy Watts

$\% \quad$ West Virginia University

$\%$

$\%$ Purpose: Aid in the development of a better controlled system

$0 \% * * * * * * * * * * * * * * * * * * * * * * * * * * * * * * * * * * * * * * * * * * * * * * * * * * * * * * * * * * * * * * * * * * * * * * * * * *$

$\% * * * * * * * * * * * * * * * * * * * * * * * * * * * * * * * * * * * * * * * * * * * * * * * * * * * * * * * * * * * * * * * * * * * * * * * * * *$

$\%$ In this section of the code constant values are defined and values are

$\%$ given to design variables that remain constant throughout the program

$0 \% * * * * * * * * * * * * * * * * * * * * * * * * * * * * * * * * * * * * * * * * * * * * * * * * * * * * * * * * * * * * * * * * * * * * * * * * *$

$\%$ Define Constants

$\mathrm{pi}=3.14159$;

sigma $=5.6704 * 10^{\wedge}-8 ; \quad \%$ Stefan-Boltzmann constant, $\mathrm{J} /\left(\mathrm{s}^{*} \mathrm{~m}^{\wedge} 2^{*} \mathrm{~K} \mathrm{~K}^{\wedge} 4\right)$

$\mathrm{g}=9.8$;

$\%$ Gravity $\mathrm{m} / \mathrm{s}^{\wedge} 2$

$\%$ Define Variables

$\mathrm{Tf}=1384$;

$\mathrm{Ta}=290$;

$\mathrm{Tc}=310$;

$\mathrm{Dn}=0.00427$;

$\mathrm{Dd}=0.0000285$;

$\%$ Furnace Temperature of Glass, $\mathrm{K}$

$\%$ Ambient Air Temperature, K

$\%$ Coil Temperature, $\mathrm{K}$

$\%$ Diameter of Nozzle, $m$

DrumV=149*18*0.00508;

$\%$ Desired Strand Diameter, $\mathrm{m}$

$\mathrm{H}=0.0508$;

$\%$ Velocity at Drum, $\mathrm{m} / \mathrm{s}$

$\mathrm{HV}=0.03$;

$\%$ Glass Height in Furnace, $\mathrm{m}$

$\operatorname{rhogf}=2.495^{*} 100^{\wedge} 3$;

$\%$ Horizontal Velocity of air, $\mathrm{m} / \mathrm{s}$

mdot $=$ rhogf*pi $/ 4 * \mathrm{Dd}^{\wedge} 2 *$ DrumV;

$\mathrm{k}=0.88$;

\%Final Glass Density, $\mathrm{g} / \mathrm{cm}^{\wedge} 3$

epsilon $=0.9$;

$\%$ Mass Flow Rate, g/s

$\%$ Thermal Conductivity of Glass, W/m*K

$\%$ Emissivity of Glass, estimated

$0 \% * * * * * * * * * * * * * * * * * * * * * * * * * * * * * * * * * * * * * * * * * * * * * * * * * * * * * * * * * * * * * * * * * * * * * * * * *$

$\%$ Adjust the following variable during post processing

\% $* * * * * * * * * * * * * * * * * * * * * * * * * * * * * * * * * * * * * * * * * * * * * * * * * * * * * * * * * * * * * * * * * * * * * * * * * *$

Tension=0.0033321; $\quad$ \%Tensile Force on the Strand 


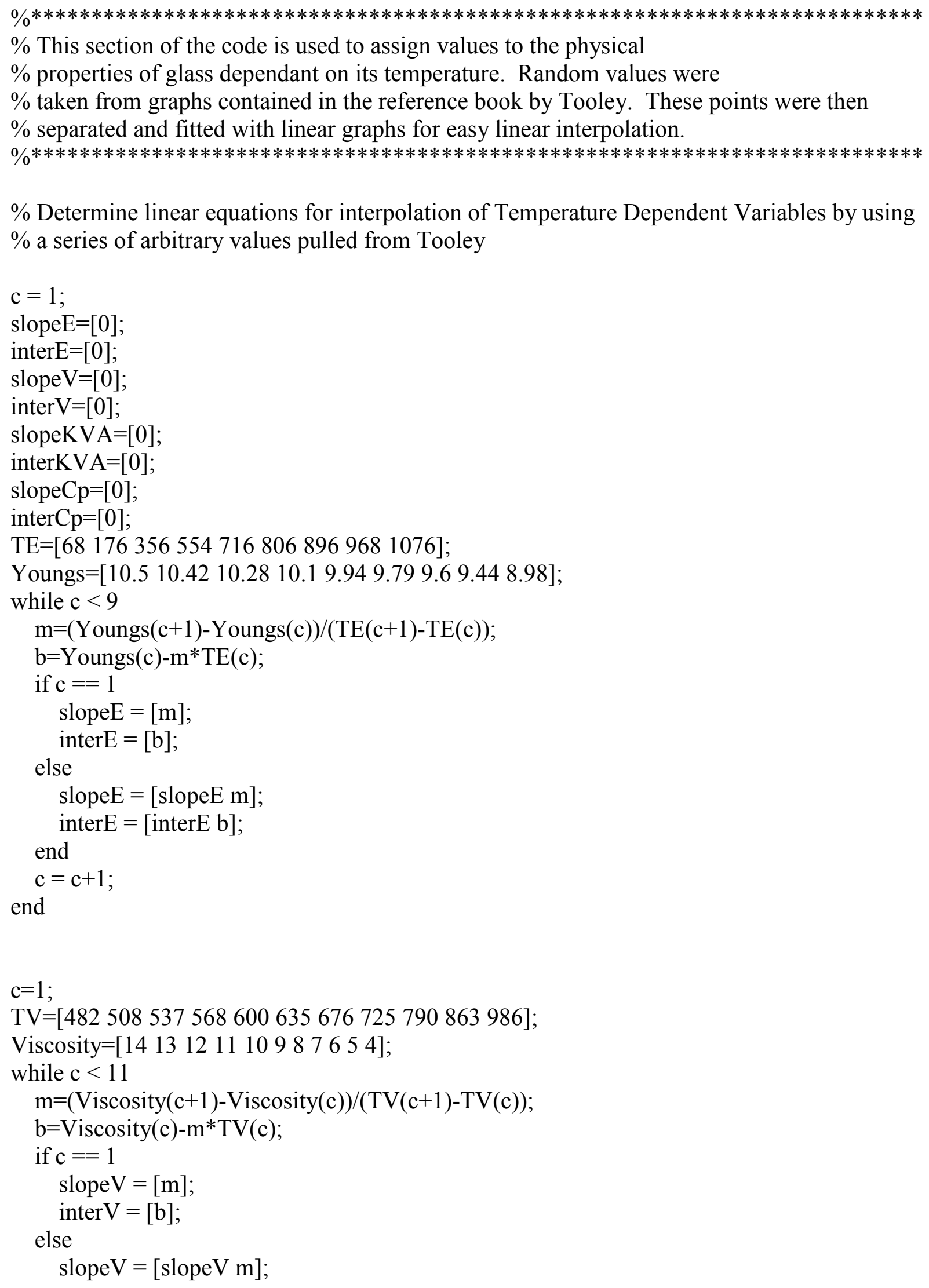




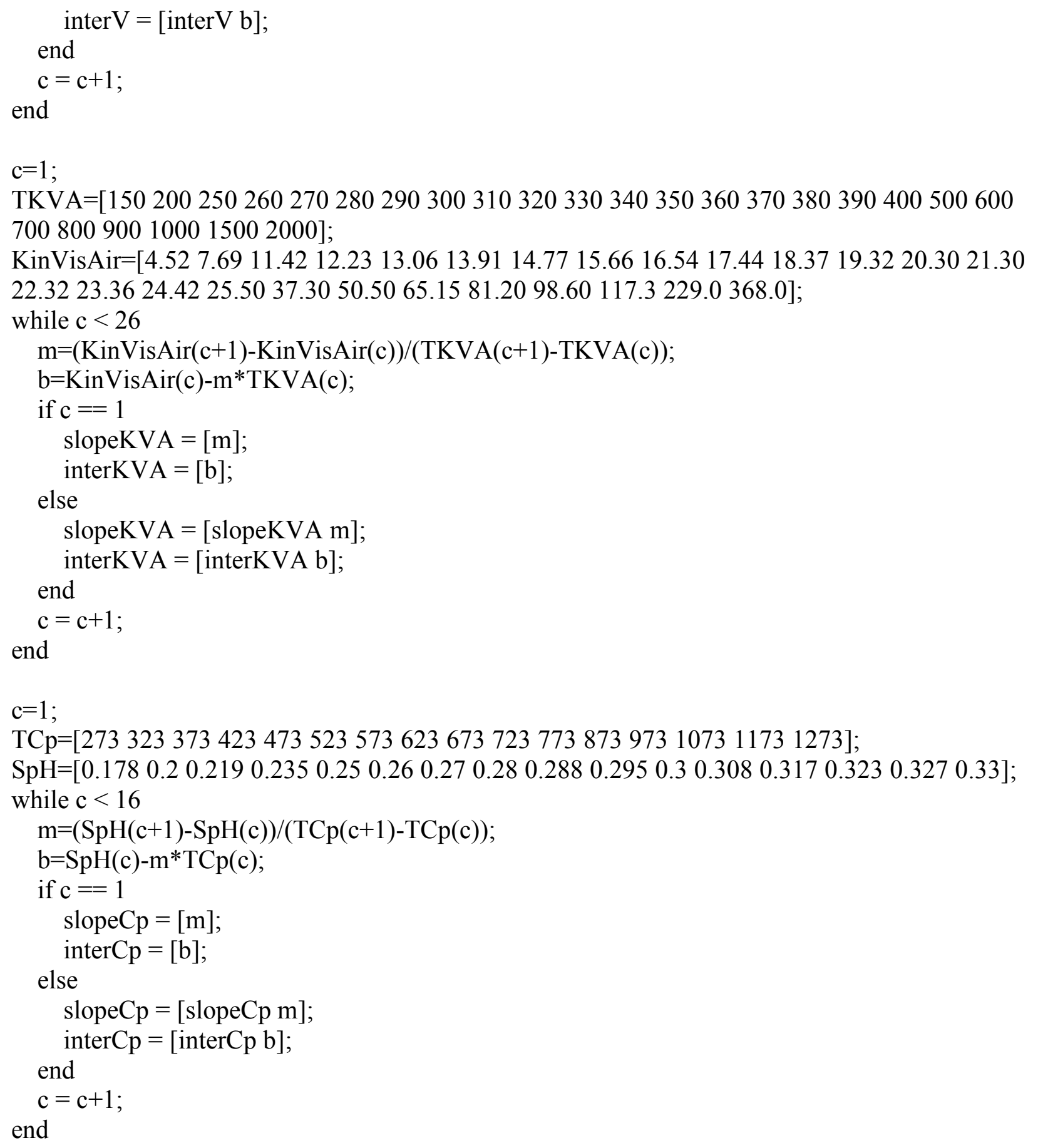


$0 \% * * * * * * * * * * * * * * * * * * * * * * * * * * * * * * * * * * * * * * * * * * * * * * * * * * * * * * * * * * * * * * * * * * * * * * * * *$

$\%$ In this section vectors are created to keep record of the values of the

$\%$ variables determined by the program at each step.

\% $* * * * * * * * * * * * * * * * * * * * * * * * * * * * * * * * * * * * * * * * * * * * * * * * * * * * * * * * * * * * * * * * * * * * * * * * * *$

$\%$ Define Initial Conditions

$\mathrm{xV}=[0] ; \quad \%$ Vector containing distance values, $\mathrm{m}$

$\mathrm{dv}=[\mathrm{Dn}] ; \quad \%$ Vector containing diameter values, $\mathrm{m}$

$\mathrm{Tv}=[\mathrm{Tf}]$; $\quad \%$ Vector containing Temperature values, $\mathrm{K}$

$\% * * * * * * * * * * * * * * * * * * * * * * * * * * * * * * * * * * * * * * * * * * * * * * * * * * * * * * * * * * * * * * * * * * * * * * * * * *$

$\%$ This section initializes variables used solely for counting. It also

$\%$ initializes the initial guess values used later in the numerical analysis

$\%$ of the temperature at the current step.

$0 \% * * * * * * * * * * * * * * * * * * * * * * * * * * * * * * * * * * * * * * * * * * * * * * * * * * * * * * * * * * * * * * * * * * * * * * * * *$

$\%$ initialize counting variables

$\mathrm{a}=1$;

ExceedsBiot $=0$

Tprofile $=[\mathrm{Tf} \mathrm{Ta}] ; \quad \%$ Sets up temperorary temp. vector

strain $1=0$;

tguess $=0$;

timeatt $=0$;

Derror $=1$;

\% $* * * * * * * * * * * * * * * * * * * * * * * * * * * * * * * * * * * * * * * * * * * * * * * * * * * * * * * * * * * * * * * * * * * * * * * * * *$

$\%$ This section of the code is the beginning of the iterative process the

$\%$ algorithm is based upon. The beginning while loop tells the code to

$\%$ continue the interations until the current distance from the nozzle value

$\%$ matches the largest desired value.

\% $* * * * * * * * * * * * * * * * * * * * * * * * * * * * * * * * * * * * * * * * * * * * * * * * * * * * * * * * * * * * * * * * * * * * * * * * * *$

while $\mathrm{xv}(\mathrm{a})<0.25$ 
$0 \% * * * * * * * * * * * * * * * * * * * * * * * * * * * * * * * * * * * * * * * * * * * * * * * * * * * * * * * * * * * * * * * * * * * * * * * * * *$

$\%$ This section of code isolates the current values for variables from the

$\%$ vectors they are stored in. It also determines values for the

$\%$ temperature dependant variables using the previously determined inline

$\%$ functions

$\% * * * * * * * * * * * * * * * * * * * * * * * * * * * * * * * * * * * * * * * * * * * * * * * * * * * * * * * * * * * * * * * * * * * * * * * * * *$

$\begin{array}{ll}\mathrm{e}=1 ; & \% \\ \text { error }=1 ; & \% \text { resets the error values } \\ \mathrm{err}=1 ; & \% \\ \mathrm{Tk}=\mathrm{Tv}(\mathrm{a}) ; & \% \text { isolates current temperature from vector } \\ \mathrm{T}=9 / 5^{*}(\mathrm{Tk}-273)+32 ; & \% \text { converts Kelvin to Fahrenheit } \\ \mathrm{D}=\mathrm{dv}(\mathrm{a}) ; & \% \text { isolates current diameter from vector } \\ \mathrm{X}=\mathrm{Xv}(\mathrm{a}) ; & \% \text { isolates current distance from vector }\end{array}$

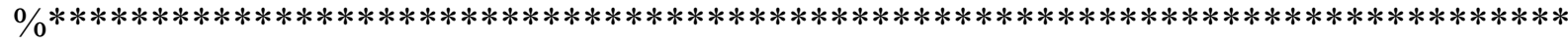

$\%$ This section of codes takes the current steps temperature values to determine values for the $\%$ temperature dependent variables using the already obtained linear equations.

\% $* * * * * * * * * * * * * * * * * * * * * * * * * * * * * * * * * * * * * * * * * * * * * * * * * * * * * * * * * * * * * * * * * * * * * * * * * *$

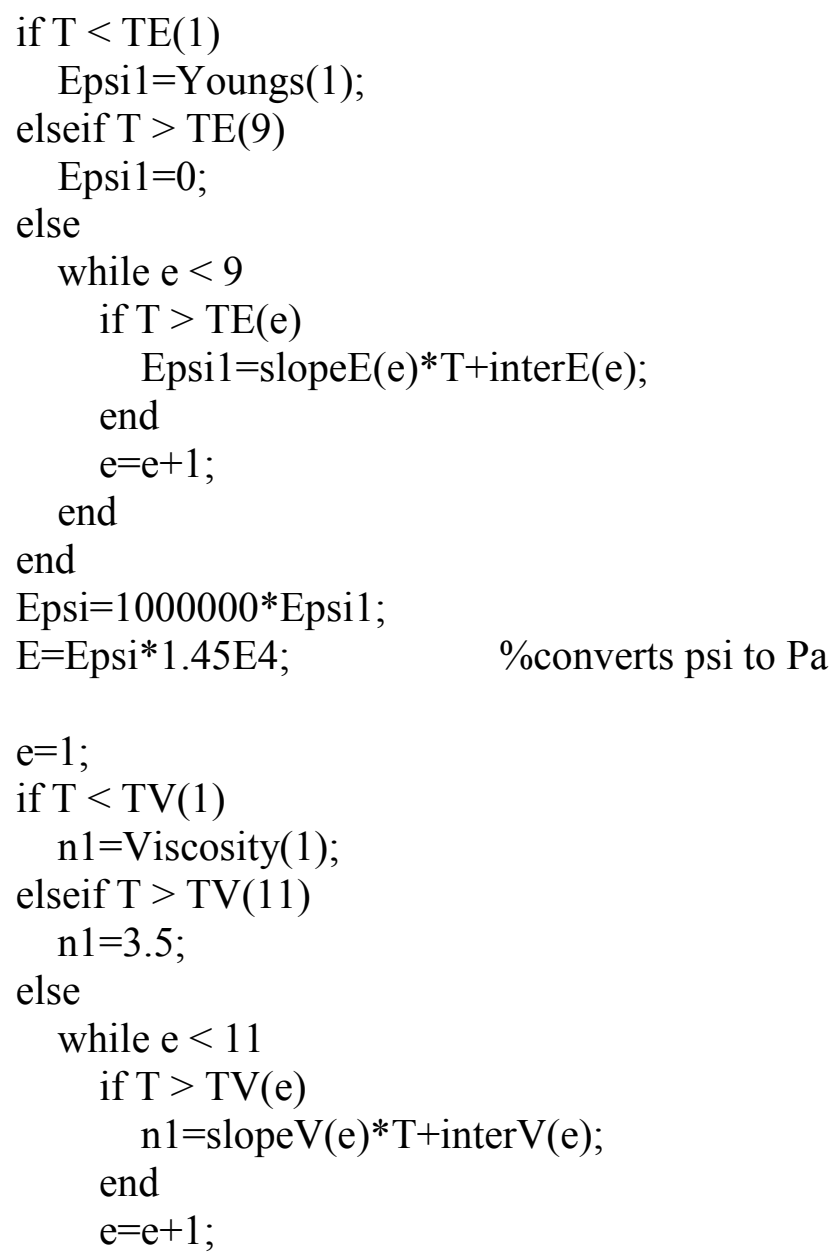




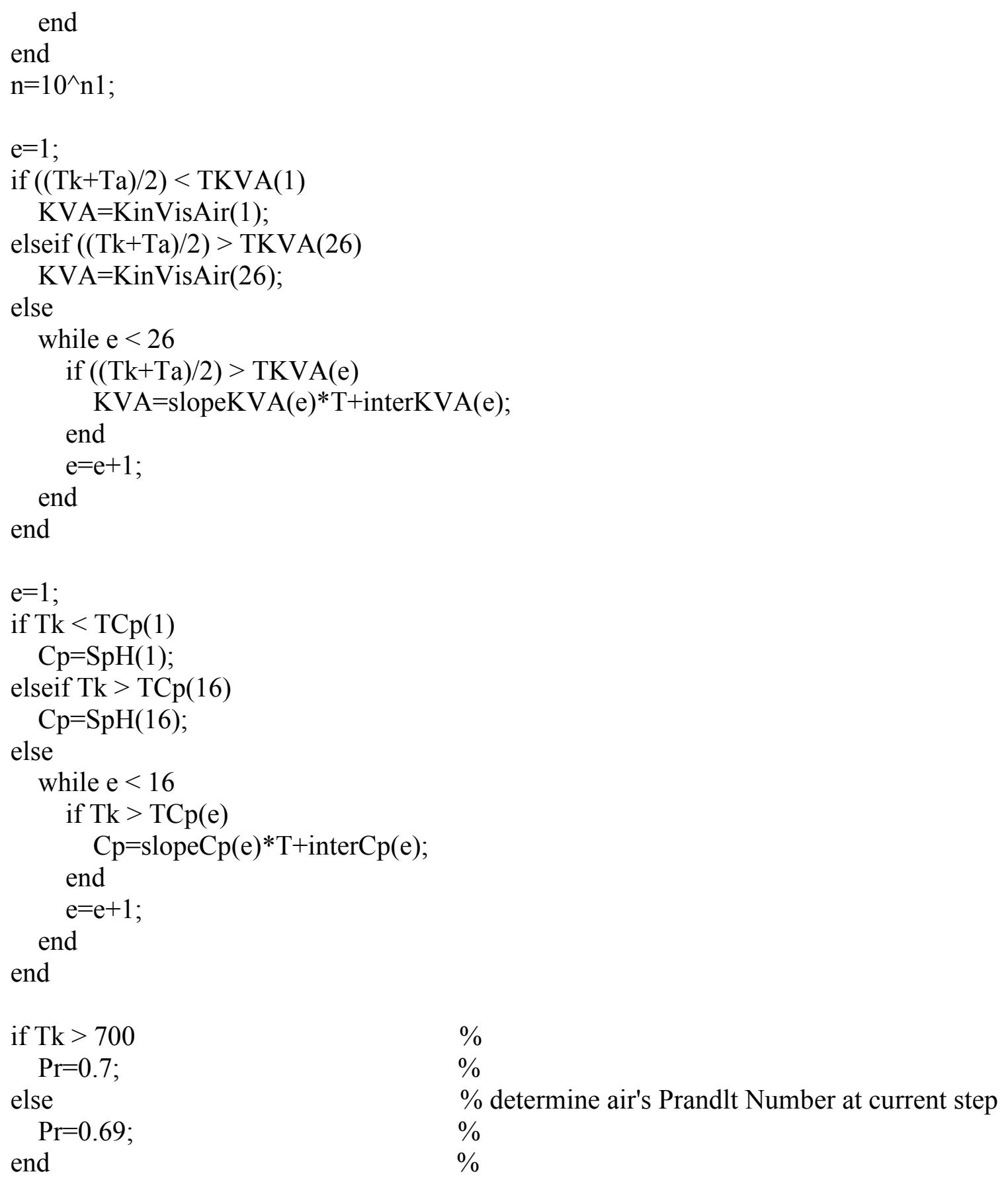




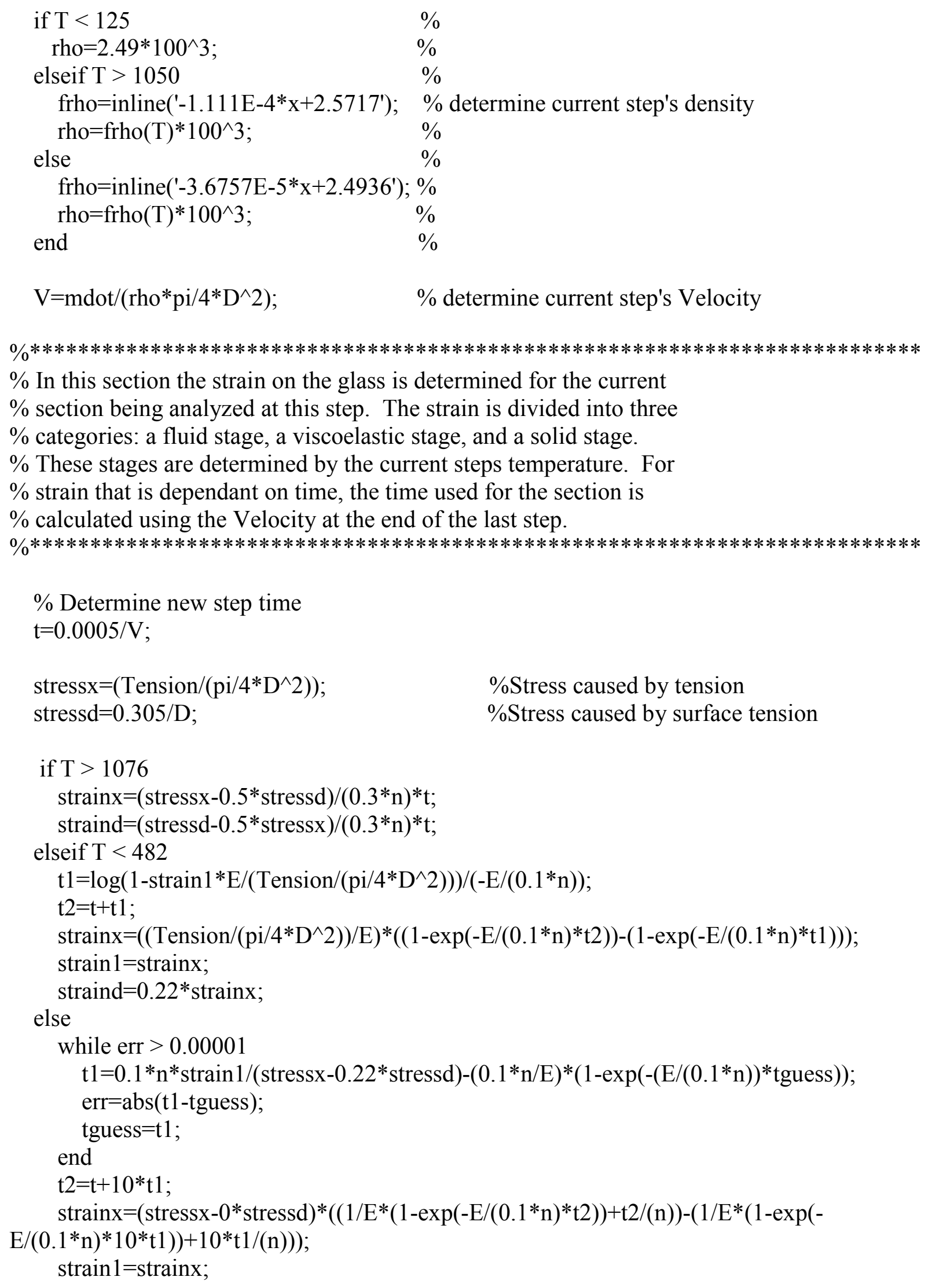


straind $=\left(0 *\right.$ stressd- $0.5^{*}$ stressx $) *\left(\left(1 / E^{*}(1-\exp (-\mathrm{E} /(0.1 * \mathrm{n}) * \mathrm{t} 2))+\mathrm{t} 2 /(\mathrm{n})\right)-\left(1 / \mathrm{E}^{*}(1-\exp (-\right.\right.$

$\mathrm{E} /(0.1 * \mathrm{n}) * 10 * \mathrm{t} 1))+10 * \mathrm{t} 1 /(\mathrm{n})))$

end

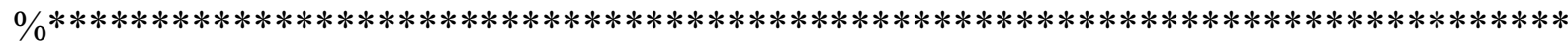

$\%$ Here a new position coordinate and a new diameter are determined using

$\%$ the strains found in the previous step. After the new values are found

$\%$ they are attached to the end of the corresponding vector.

\% $* * * * * * * * * * * * * * * * * * * * * * * * * * * * * * * * * * * * * * * * * * * * * * * * * * * * * * * * * * * * * * * * * * * * * * * * * *$

$\%$ Determine new position coordinate

$\mathrm{X}=\mathrm{Xv}(\mathrm{a})+0.001+0.001 *$ strain $\mathrm{x}$

$\%$ Determine new diameter

if $\mathrm{Tk}>482$

$\mathrm{D}=\mathrm{D}+\mathrm{D}$ *straind;

else

Volume $=0.001 * \mathrm{pi} / 4 *\left(\mathrm{dv}(\mathrm{a})^{\wedge} 2\right)$;

$\mathrm{D}=\operatorname{sqrt}($ Volume $/(\mathrm{pi} / 4 *(\mathrm{X}-\mathrm{xv}(\mathrm{a}))))$;

end

\% Update Vectors to Contain new values

$\mathrm{XV}=[\mathrm{xV} \mathrm{X}]$;

$d v=[d v D]$;

if $\mathrm{a}==1$

$\mathrm{Ev}=[\mathrm{E}]$

$\mathrm{nv}=[\mathrm{n}]$;

else

$\mathrm{Ev}=[\mathrm{Ev} \mathrm{E}]$;

$\mathrm{nv}=[\mathrm{nv} \mathrm{n}]$;

end

$\%$ Add counter to array size counter $\mathrm{a}=\mathrm{a}+1$; 
$\% * * * * * * * * * * * * * * * * * * * * * * * * * * * * * * * * * * * * * * * * * * * * * * * * * * * * * * * * * * * * * * * * * * * * * * * * * *$

$\%$ This section of code deals primarily with the temperature of the glass.

$\%$ The heat transfer variables are first determined using the newly found

$\%$ physical values. The strand is divided into two sections when dealing

$\%$ with heat transfer: an area contained in a radiative hood, and a section

$\%$ open to higher convective heat transfer. An interior loop is used to

$\%$ perform an iterative numerical method in both sections.

$0 \% * * * * * * * * * * * * * * * * * * * * * * * * * * * * * * * * * * * * * * * * * * * * * * * * * * * * * * * * * * * * * * * * * * * * * * * * *$

$\%$ Determine New Nodal Temperature

$\operatorname{Davg}=(\operatorname{dv}(\mathrm{a})+\operatorname{dv}(\mathrm{a}-1)) / 2$;

$\mathrm{Re}=\mathrm{HV}^{*} \mathrm{Davg} / \mathrm{KVA}$;

if $\mathrm{xv}(\mathrm{a})<0.1$

alpha $=x v(a) * 10$;

$\mathrm{Re}=$ alpha*HV*Davg/KVA;

end

$\mathrm{Nu}=1 /\left(0.8237-\log \left((\operatorname{Re} * \operatorname{Pr})^{\wedge} 0.5\right)\right)$;

$\mathrm{h}=(\mathrm{k} / \mathrm{Davg})^{*} \mathrm{Nu}$;

$\mathrm{Bi}=\mathrm{h} * \operatorname{Davg} /(4 * \mathrm{k})$;

if $\mathrm{Bi}>0.2$

ExceedsBiot $=$ ExceedsBiot $+1 ; \quad \%$ Tests for large Biot Numbers

end

$\%$

$\mathrm{L}=\mathrm{xv}(\mathrm{a})-\mathrm{xv}(\mathrm{a}-1) ; \quad \quad \%$ Determine effective length for transfer

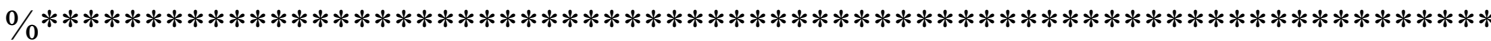

$\%$ This section of code determines the shape factor using Hottel's

$\%$ String Rule as described in the technical approach section

\% $* * * * * * * * * * * * * * * * * * * * * * * * * * * * * * * * * * * * * * * * * * * * * * * * * * * * * * * * * * * * * * * * * * * * * *$

Wavg=.20475;

$\mathrm{AD}=\operatorname{sqrt}\left(\operatorname{Wavg}^{\wedge} 2+(\mathrm{xv}(\mathrm{a}-1)-0.1)^{\wedge} 2\right)$

$\mathrm{BC}=\operatorname{sqrt}\left(\mathrm{Wavg}^{\wedge} 2+\mathrm{xv}(\mathrm{a})^{\wedge} 2\right)$;

$\mathrm{AC}=\operatorname{sqrt}\left(\operatorname{Wavg}^{\wedge} 2+\mathrm{xv}(\mathrm{a}-1)^{\wedge} 2\right)$;

$\mathrm{BD}=\operatorname{sqrt}\left(\operatorname{Wavg}^{\wedge} 2+(\operatorname{xv}(\mathrm{a})-0.1)^{\wedge} 2\right)$;

$\mathrm{F}=1 /(2 * \mathrm{~L}) *(\mathrm{AD}+\mathrm{BC}-\mathrm{AC}-\mathrm{BD})$;

while error $>0.00005$

$\mathrm{T} 1=\mathrm{Tprofile}(1)$

$\mathrm{T} 2=\mathrm{Tprofile}(2)$;

Asur=pi*Davg*L;

alpha $=x v(a) * 10$ 


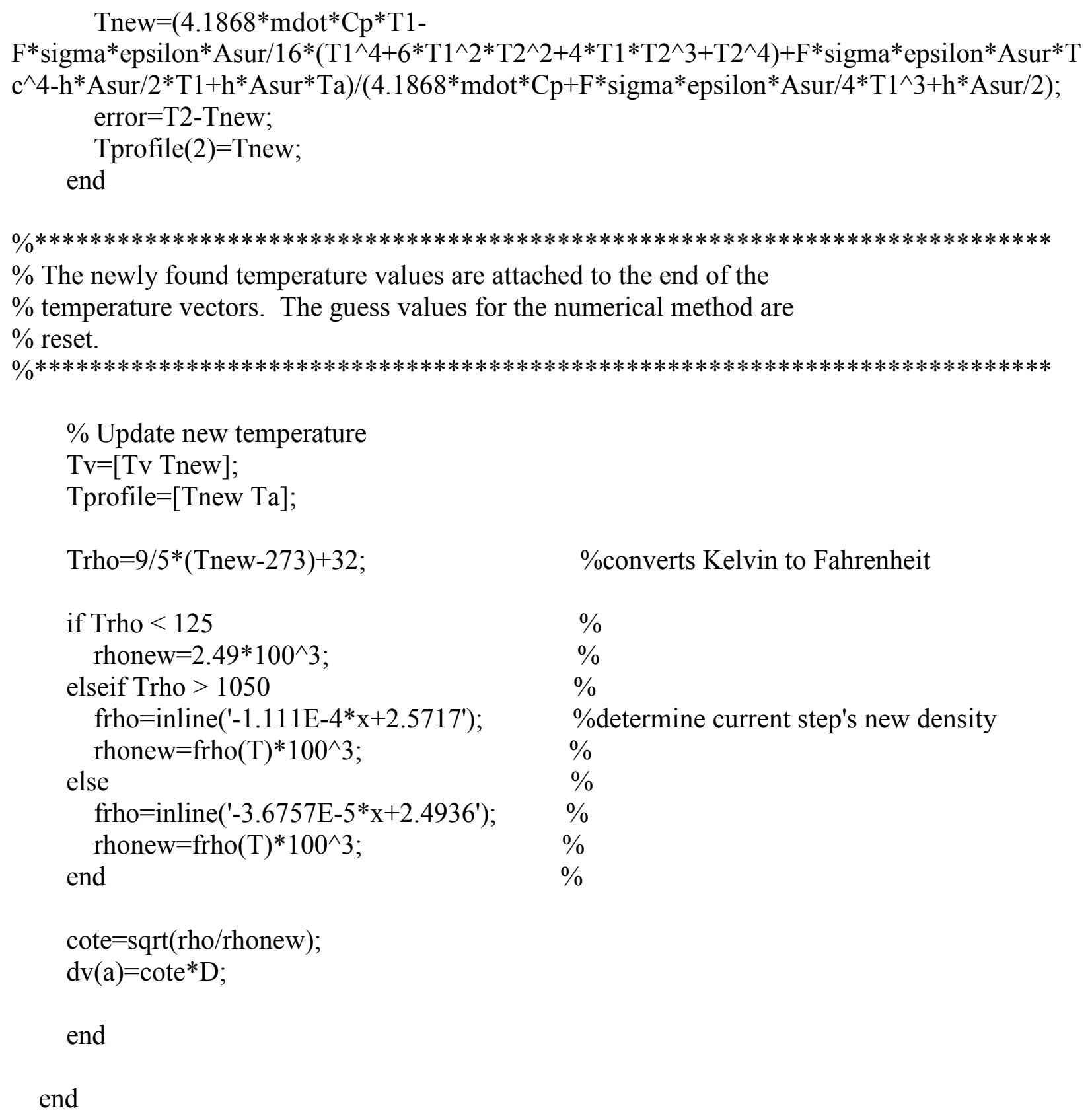




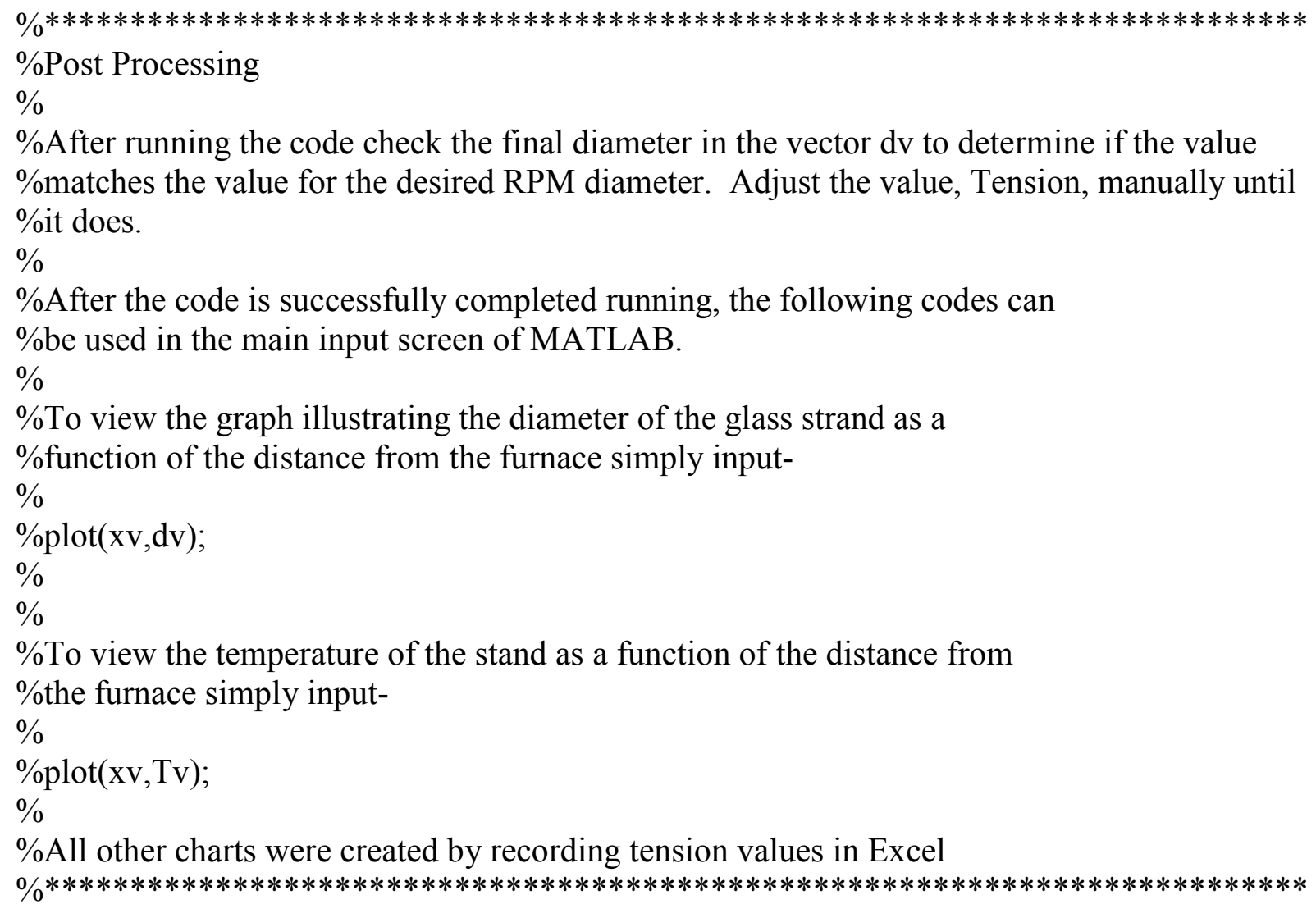




\section{Appendix 3 Deriving Viscoelastic Equations}

The following is a closer look at deriving the characteristic equations of the Kelvin and Maxwell models. This work is a paraphrased collection of methods used in the book by Shames and Cozzarelli [5].

The Maxwell model consists of a spring in series with a dashpot. In order to derive a behavioral equation it is important to start with basic equations. Since the two elements are in series, it is true that the stress in the spring is equal to the stress in the dashpot, and the total strain is equal to the strain of the spring plus the strain of the dashpot, hence [5]:

$$
\begin{gathered}
\tau=\tau_{S}=\tau_{D} \\
\varepsilon=\varepsilon_{S}+\varepsilon_{D} \\
\dot{\varepsilon}=\dot{\varepsilon}_{S}+\dot{\varepsilon}_{D}
\end{gathered}
$$

The relationships between stress and stain in the individual elements are also known as functions of the Modulus of Elasticity and the Viscosity and can be given as follows [5]:

$$
\begin{gathered}
\varepsilon_{S}=\frac{\tau_{S}}{E} \\
\dot{\varepsilon}_{S}=\frac{\dot{\tau}_{S}}{E} \\
\dot{\varepsilon}_{D}=\frac{\tau_{D}}{\eta}
\end{gathered}
$$

Finally, by combining all equations the governing formula for the Maxwell element can be given as [5]:

$$
\dot{\varepsilon}=\frac{\dot{\tau}}{E}+\frac{\tau}{\eta}
$$


The Kelvin model combines the spring and dashpot in parallel and the governing equation can be found in a similar fashion to the Maxwell model except now the strain in the spring element is equal to the strain in the dashpot, and the total stress is the sum of the stresses in the spring and dashpot [5].

$$
\begin{gathered}
\tau=\tau_{S}+\tau_{D} \\
\varepsilon=\varepsilon_{S}=\varepsilon_{D}
\end{gathered}
$$

From before [5]:

$$
\begin{gathered}
\varepsilon_{S}=\frac{\tau_{S}}{E} \\
\dot{\varepsilon}_{D}=\frac{\tau_{D}}{\eta}
\end{gathered}
$$

Combining these equations we can finally arrive at [5]:

$$
\tau=\eta \dot{\varepsilon}+E \varepsilon
$$

To better understand the behavior of these two viscoelastic models, the creep test and the relaxation test can be applied. The creep test applies constant stress to the system and uses the behavior equation to determine the strain on the model as a function of time. Likewise the relaxation test applies a constant strain on the model to determine the stress in the system as a function of time.

Before it is possible to illustrate the behavior of the two tests on the different models, it is necessary to have a basic understanding of singularity functions starting with the unit step function. The unit step function in time is denoted as $\left[\mathrm{u}\left(\mathrm{t}-\mathrm{t}_{0}\right)\right]$ and defined as $[5]$ :

$$
\begin{aligned}
& {\left[\mathrm{u}\left(\mathrm{t}-\mathrm{t}_{0}\right)\right]=0 \text { for } \mathrm{t}<\mathrm{t}_{0}} \\
& {\left[\mathrm{u}\left(\mathrm{t}-\mathrm{t}_{0}\right)\right]=1 / 2 \text { for } \mathrm{t}=\mathrm{t}_{0}} \\
& {\left[\mathrm{u}\left(\mathrm{t}-\mathrm{t}_{0}\right)\right]=1 \text { for } \mathrm{t}>\mathrm{t}_{0}}
\end{aligned}
$$


The unit step function is used primarily to illustrate a constant input over the time period $\left(\mathrm{t}-\mathrm{t}_{0}\right)$. An important property of the unit step function is how it acts during integration. The integral of a function of time $f\left(t^{\prime}\right)$ multiplied by the unit step function becomes [5]:

$$
\int_{-\infty}^{t} f\left(t^{\prime}\right)\left[u\left(t-t_{0}\right)\right] d t^{\prime}=\left[u\left(t-t_{0}\right)\right] \int_{t_{0}}^{t} f\left(t^{\prime}\right) d t^{\prime}
$$

The derivative with respect to time of the unit step function is known as the Dirac Delta function, $\left[\delta\left(\mathrm{t}-\mathrm{t}_{0}\right)\right]$. It is important to note the integration of the Dirac Delta function multiplied by $f\left(t^{\prime}\right)$ becomes $[5]$ :

$$
\int_{-\infty}^{t} f\left(t^{\prime}\right)\left[\delta\left(t^{\prime}-t_{0}\right)\right] d t^{\prime}=f\left(t_{0}\right)\left[u\left(t-t_{0}\right)\right]
$$

Similar to the Dirac Delta function is its derivative with respect to time known as the Doublet Function, $\left[\eta\left(t-t_{0}\right)\right]$ and the integration of it multiplied by $f\left(t^{\prime}\right)$ becomes [5]:

$$
\int_{-\infty}^{t} f\left(t^{\prime}\right)\left[\eta\left(t^{\prime}-t_{0}\right)\right] d t^{\prime}=f(t)\left[\delta\left(t-t_{0}\right)\right]-\dot{f}\left(t_{0}\right)\left[u\left(t-t_{0}\right)\right]
$$

Now it is possible to examine the creep and relaxation tests on the two models, starting with the Maxwell model (a spring and dashpot in series). Recall that the behavior equation for the Maxwell model was [5]:

$$
\dot{\varepsilon}=\frac{\dot{\tau}}{E}+\frac{\tau}{\eta}
$$

When performing the creep test, constant stress is placed on the model to examine the strain as a function of time, therefore [5]:

$$
\tau=\tau_{0}[\mathrm{u}(\mathrm{t})] \text {, since } \mathrm{t}_{0}=0 \text { in this case, i.e. } \tau=\tau_{0} \forall \mathrm{t}>0
$$

Substitution into the behavior equation gives [5]:

$$
\dot{\varepsilon}=\frac{\tau_{0}}{E} \frac{d}{d t}[u(t)]+\frac{\tau_{0}}{\eta}[u(t)]
$$




$$
\dot{\varepsilon}=\frac{\tau_{0}}{E}[\delta(t)]+\frac{\tau_{0}}{\eta}[u(t)]
$$

Integrating gives [5]:

$$
\varepsilon(t)=\tau_{0}\left(\frac{1}{E}+\frac{t}{\eta}\right)[u(t)]
$$

Like the creep test, the relaxation tests uses the behavior equation except this time strain is kept constant.

$$
\varepsilon=\varepsilon_{0}[\mathrm{u}(\mathrm{t})]
$$

Hence the behavior equation becomes [5]:

$$
\begin{gathered}
\varepsilon_{0} \frac{d}{d t}[u(t)]=\frac{\dot{\tau}}{E}+\frac{\tau}{\eta} \\
\dot{\tau}+\frac{E}{\eta} \tau=\varepsilon_{0} E[\delta(t)]
\end{gathered}
$$

Multiplying by an integrating factor and then integrating from $\mathrm{t}=0$ to $\mathrm{t}=\mathrm{t}$ the equation becomes [5]:

$$
\tau(t)=\varepsilon_{0} E e^{-(E / \eta) t}[u(t)]
$$

The Kelvin model behaves very differently than the Maxwell model. Here the elastic element and viscous element are in parallel, and the behavior equation is [5]:

$$
\tau=\eta \dot{\varepsilon}+E \varepsilon
$$

Applying the creep test on the Kelvin model gives [5]:

$$
\dot{\varepsilon}+\frac{E}{\eta} \varepsilon=\frac{\tau_{0}}{\eta}[u(t)]
$$

Like the relaxation test for the Maxwell element the equation needs to be multiplied by an integrating factor and then integrated from $\mathrm{t}=0$ to $\mathrm{t}=\mathrm{t}$ 


$$
\varepsilon(t)=\frac{\tau_{0}}{E}\left(1-e^{-(E / \eta) t}\right)[u(t)]
$$

Applying the relaxation test to the Kelvin model shows that theoretically the Kelvin model never relaxes [5].

$$
\tau(t)=\varepsilon_{0}\{\eta[\delta(t)]+E[u(t)]\}
$$

